\title{
HILGARDIA
}

A Journal of Agricultural Science Published by the California Agricultural Experiment Station

\section{PRICING EFFICIENCY IN THE MANUFACTURED DAIRY PRODUCTS INDUSTRY}

\author{
JAMES B. HASSLER
}

UNIVERSITY OF CALIFORNIA - BERKELEY, CALIFORNIA 
This investigation is concerned with the price results of the United States dairy products industry from the plant level to the wholesale level. Particular emphasis is placed on intermarket product prices in some major cities, on relative net prices of products at processing plants, and the connection between the latter values and producer prices for milk. Although price relationships will be the core of the study, quantitative volume changes will sometimes be employed to show directional reaction to price disequilibria.

The general framework of the study is the abstract, perfectly competitive market in space, form, and time. Quantitative appraisal is made of the actual price results of the industry with respect to these aspects, with only minor emphasis on the short-period temporal problem. 


\section{H I $\quad$ L G G A R $\quad$ D D \\ the California Agricultural Experiment Station}

VoL. 22

AUGUST, 1953

No. 8

\section{PRICING EFFICIENCY IN THE MANUFACTURED DAIRY PRODUCTS INDUSTRY, ${ }^{1,2}$}

\section{JAMES B. HASSLER ${ }^{3}$}

\section{Introduction}

FOUR IMPORTANT FACTORS together influence the complex marketing and pricing system of the manufactured dairy products industry. First, is the fact that the supply areas for manufacturing grade milk are not geographically coördinated, in a quantitative sense, with the dense population areas. It is not the density of population itself that is relevant but the effective demand caused by the incomes and tastes of the people. Second, manufacturing grade milk is a nonspecialized raw material to the industry. A fairly large number of alternative utilizations exist from which entrepreneurs may choose, depending on the economic advantages of each. Third, manufactured dairy products are relatively nonperishable. This permits long-distance movements and long-period storage. Fourth, this factor involves the weight of the final products when a given quantity of milk is employed in each of the alternative uses. Significant differences occur in these transformations. If the weight of the products made from a given quantity of milk is small, these products must have a relatively high average net value per unit of weight compared with products that have larger weight per unit of milk in a different utilization when the two uses are effectively competing for the raw milk supply. The concentration of net value into smaller weight alsu permits, in an economic sense, the extension of the distance such products move in competition with other supply areas.

Indicators of advantages and disadvantages. In a real environment in which the four factors influence those who make decisions on production, utilization, and distribution, the complex system of relative prices would function as indicators of situational advantages and disadvantages. A simultaneous duality exists between decisions and prices, that is, the decisions generate the prices upon which further decisions are made. This element of

${ }^{1}$ Received for publication June 6, 1952.

2 This paper is in part a development of work done on the program of the Western Regional Dairy Marketing Project under authority of the Agricultural Marketing Act of 1946. The author wishes to acknowledge the important assistance and review generously contributed by Professors R. G. Bressler, Jr., and D. A. Clarke, Jr.

${ }^{3}$ Lecturer in Agricultural Economics and Assistant Agricultural Economist in the Experiment Station and on the Giannini Foundation, Berkeley. 
duality leads to a more or less efficient, self-correcting, productive system, depending on how much time elapses between decisions and price results. In the absence of noncompetitive buying and selling, the net over-all result would tend toward an organization of supply and distribution, in response to demand intensities, that provided the greatest possible economic satisfaction with a minimum of cost in the form of unsatisfied alternatives which the employed resources could have supplied. The significance of this statement rests on the normative properties of a competitive system that equates marginal social and individual economic values. Conflicts may arise between the competitive system and noneconomic values existing in a given society so that, from the total welfare point of view, a competitive system may not be desirable even if feasible. Certain amounts of permissive monopoly or monopsony may be more efficient with respect to total values.

Limitation of scope. A full analysis of how well the manufactured dairy products industry achieves operational efficiency would require an intensive econometric study based on interdependent localized data at all levels from farm supply to home consumption. This study does not anticipate solving such a formidable problem. Its scope is much more limited. Briefly stated, this investigation will concern itself with the price results of the United States manufactured dairy products industry from the plant level to the wholesale level. Particular emphasis will be placed on intermarket product prices in some major cities, on relative net prices of products at processing plants, and the connection between the latter values and producer prices for milk. Geographically, the Midwest and selected states of the Western Region will be used to stress important interdependencies. A simplified model will be employed as a comparative base for evaluating actual price results of the past. The entire project is devoted to such comparisons. However, the model is timeless and, with assumed modification (changes in product standards, costs of processing due to technological changes, et cetera), usable for future predictions.

Although price relationships will be the core of the study, quantitative volume changes will sometimes be employed to show directional reaction to price disequilibria. Shifting geographical distributions of demand relative to supply have a significant effect on issues of comparative advantage and regional specialization in production. Such shifts may have a marked effect on intermarket price relationships as the areas change from surplus to deficit conditions of supply over demand. Shipment and receipt volumes may corroborate these changes.

Framework for study. The general framework is the abstract, perfectly competitive market in space, form, and time. Quantitative appraisal will be made of the actual price results of the industry with respect to these aspects, with only minor emphasis on the temporal problem. In order to avoid unnecessary duplication, a detailed statement of the problem and the approach will not be made here. Section III is devoted to such specification in detail.

Probable values of study. What values may result from such an analysis as developed herein? Broadly speaking, it should provide foundational information for policy decisions, both public and private. On the private level. it should enable entrepreneurs to choose wisely between alternative utiliza- 
tion and distribution possibilities when facilities for doing so exist. On an area basis (due to interarea competition), it should present facts pointing to comparative advantage in specialization. From the public point of view, partial bases for price-support policy, production-shifting policy (using prices as motivators), and legislative-control policy should be developed. In effect, such an analysis should uncover data through which wiser decisions can be made by individuals and groups as the latter operate within the constraints of the general rules developed for the entire society.

The value of economic efficiency research, it should be stressed, ultimately rests on the relationship of such efficiency to general welfare. When an economic activity has been shown to be inefficient, the proof is only relative to the adequacy of the standard of comparison. The complex interdependencies of economic and noneconomic social values make it extremely difficult to choose a correct standard by which to judge economic operations for efficiency. The following section presents a limited discussion of the difficulties encountered in ascertaining measurements of economic inefficiencies and their welfare implications.

\section{Productive Efficiency, Pricing Efficiency, and Social Values}

Social structure. The structure of a society is composed of three interdependent elements, namely, people, resources, and a set of rules of behavior. In the area of habitation, resources of varying availability and finite economic limitations are found. These resources include the available natural resources, the inherited wealth of man-made capital forms from the past, the accumulated knowledge of technological processes, and the physical and mental capabilities of the people. The rules (written and/or unwritten) of behavior are a body of sanctioned and enforced controls that set boundaries for individual actions. Among these rules are found the mechanisms whereby individuals can, in a direct or indirect manner, employ the privilege and right of having their opinions bear on current decisions of group scope. Channels are also available, in varying degrees, to permit revisions and changes in the rules over time. These procedural rules give dynamic characteristics to a society.

Economic efficiency and social values. The full set of rules of behavior adopted by a society strongly influence the economic and intangible (aspects of freedom) values accruing to it. Efficiency for the whole system must take into account both areas of welfare. Insofar as features in the rules which are directed toward realizing the intangible values may not be perfectly compatible with efficiency in the economic sector based on an arbitrary system, the research economist must exercise caution in postulating models for testing the relative efficiency of the existing economic system. The basic model for comparison must be admissible under the rules and must reflect the physical relationships of economies of scale relative to demand and spatial and temporal immobility of resources. For any complete system of economic activities, these facts would probably rule out the perfectly competitive model. It is quite probable that an efficient social system would have sizable volumes of monopolistic and monopsonistic activity in the economic segment because of the intangible satisfactions derived from those rules indirectly 
fostering these distortions. The optimum economic model under these conditions would be highly complex and mixed and probably not easily specified by inductive reasoning.

Although there is high interdependence between the given set of rules of a society and the economic system, the rules are frequently sufficiently broad and general so that wide variation of organizational form, resource allocation, and output can result. The task of analyzing the complete system for efficient performance may be impossible. Still, this does not invalidate such an analysis for an industry that represents a small segment of the complete system. Such an industry may possess features which closely approximate a model having desirable implications, with the model being a reasonably admissible form under the rules that impinge on that sector.

The supposition is, of course, that the activities of the industry are efficient when the actual realized results conform closely with those postulated in the model. Further, when these conditions hold true, it can be assumed that the industry's performance is contributing in a positive manner to social welfare, even though other sectors of the economic system which either supply services to or receive output from this particular industry may be inefficient. When other sectors of the system are operating inefficiently, it is to be expected that the composition and level of inputs used and outputs produced by the given industry being studied will not have values equal to those which would result if the whole system were efficient. But in a partial analysis of this nature, the latter fact need not be ascertained even if it were possible. The important aspect for analysis is the internal performance characteristics of the industry. If these are efficient and compatible with the norm, then the industry can be expected to continue to operate in a desirable way under changing external conditions, provided these latter changes do not nullify the normative model.

Characteristics of economic activities. Associated with the volumes of final outputs from an economic activity are two time dimensions, namely, a rate and a duration interval. This is true for both the firm and industry. On the demand side, the same two dimensions exist. In both cases the duration intervals may be discontinuous and may be forced, by external circumstances, into incompatible phases and lengths. Production and consumption rates will differ in such circumstances and a fluctuating storage operation will be undertaken to balance the volumes over time. The production and consumption of many agricultural products fall in this category. This situation does not occur for directly consumed goods and services, such as electricity, for which production and consumption are essentially simultaneous.

Most economic activities are multistage processes in which intermediate product forms are produced and consumed in the development of final output forms. What was said in the last paragraph is fully applicable to these internal aspects of a firm's operations. If the duration intervals for all the stages must be the same, then the various optimum rates may be made compatible by increasing the number of basic units at the necessary stages. Certain external supply conditions and cases of multiple intermediate outputs with limited varying proportions at a given stage may lead to situations in which the above type of adjustment in firm structure is impossible. 
Either some loss in efficiency must be accepted at one or more stages, or the phases and duration intervals of the various stages must be altered (if possible) with a consistent internal storage operation undertaken between the necessary stages. The alternative which would be followed is that one which had most advantage in profit comparisons.

Although much could be written about the connection between the various dependent production functions at each stage and the over-all function relating externally supplied inputs and internally produced final outputs, the discussion will go directly to the latter since it has principal relevance to the present development.

Productive efficiency. How would one measure the productive efficiency of a competitive industry at a given point in time? A knowledge of three facts would be basic, that is, demand schedules for industry outputs, shortand long-run supply schedules for inputs used by the industry, and the physical production function(s) which is (are) technologically feasible. With this information, short- and long-run comparisons could be made, although the magnitude of the task when significant quality variations for inputs exist between firms (especially the less variable forms) would make it impractical.

At the given point in time, a cross-sectional picture of every firm in the industry would be made, accepting the durable structure of each organization. For each of these firms, the optimum variable short-run adjustment schedule at going prices for inputs and outputs would be developed from their individual production functions. Should every firm be operating at that point on the adjustment schedule which was uniquely its optimum (producing maximum variable cost coverage), then the industry would be operating at 100 per cent short-run efficiency and the going prices would be consistent prices. Should a significant number of firms be operating at nonoptimum points (either due to an inefficient cost rate, output value rate, cr both), then particular, quantitative efficiency measurements could be made for each individual firm. These measurements would be conditional since industry effects on input and output prices following adjustments by the obviously inefficient firms would undoubtedly prove that all firms were inefficient in comparison with points which would result when a perfect shortrun state of efficiency was reached by the industry. There appears to be no unique method of aggregating these conditional measurements into a quantitative industry measurement.

As was indicated in the last paragraph, 100 per cent short-run productive efficiency for the industry is synonymous with short-run equilibrium for every firm in the industry. Such a state implies a specific set of physical quantities of inputs used and outputs produced by the industry. Similarly, if one knew the optimum long-run structure for each firm and the rate at which fixed costs should be covered in the short-run period of time, he could determine whether each firm and the industry were operating at 100 per cent long-run efficiency. This concept of productive efficiency is synonymous with long-run equilibrium for every firm and, consequently, for the industry and requires that: (1) the firms are optimum in structure ; 2 ) they are operating at their uniquely optimum rates; and (3) the total number of firms 
is such as to result in total value of outputs equal to total cost of inputs for each and every firm. Value and cost are measured in a rate sense.

Equilibrium and consistent prices. If the industry is operating at 100 per cent long-run efficiency, a specific set of physical quantities for inputs and outputs results. Furthermore, a consistent set of prices for inputs and outputs is an essential part of this long-run equilibrium state. Consequently, if one knows certain relevant interrelationships within this consistent set of long-run equilibrium prices, he can tell by comparisons when the industry is operating inefficiently with respect to quantities without evaluating the quantity aspects of the productive system. Perfect pricing efficiency is an essential part of productive efficiency and is synonymous with price interrelationships that exist among those prices produced by the industry when it operates in long-run equilibrium. It indicates perfection in the relationship between decisions and price results.

A simplified price model. The remaining portion of this section will be devoted to designating the relevant price interrelationships to be used in studying the pricing efficiency of a competitive industry. It will be primarily introductory since in the next section a thorough discussion will be given especially oriented toward the manufactured dairy products industry.

Let an industry be specified on the basis of a grouping of all firms utilizing a given homogeneous raw material, $X$. This raw material can be processed into only two individually homogeneous final products, $A$ and $B$. With respect to weight, products $A$ and $B$ are quantitatively related in production to a unit of $X$ by two constants, $k_{\mathrm{A}}$ and $k_{\mathrm{B}}$. Assuming that the inputs other than $X$ are nonspecialized to the industry, the crucial competition among the firms will center on $X$. Consequently, payments by firms to suppliers of $X$ will tend to be of a residual nature after payments to other factors have been made. Geographically, at each point where $A$ and $B$ can be produced with equal economic advantage, a single price for $X$ will exist and f.o.b. plant prices for $A$ and $B$ will be such as to permit the payment of this price after all other factors have been paid. It is obvious that these prices will be related to each other through the constants, $k_{\mathrm{A}}$ and $k_{\mathrm{B}}$, and the value of payments to the other factors expressed on a per-unit basis with respect to $A$ and $B$. Competitive firms will attempt to reduce payments to the other factors by careful utilization, there being no opportunity for price bargaining. This aspect of pricing efficiency may be called form efficiency and is directly manifested in relative net prices at those geographic points where $A$ and $B$ can be produced with equal economic advantage.

Products $A$ and $B$ will be produced at various geographic points that are not coincident with consumption points. Spatial movements are required and such movements involve positive costs. These costs necessitate geographic differences in prices for each product. Such variation should perfectly reflect the transfer costs. This aspect of pricing efficiency may be called geographic efficiency.

External to the processing segment of the industry, certain biological and economic forces might lead to divergent time patterns of production and consumption rates. Costs involved in attempting to secure equal current rates might be prohibitive. In these cases a storage activity would be inter- 
jected, based on economic considerations, between the production stage and the consumption stage. A certain amount of cost would be involved in this step, and temporal price variations should perfectly reflect such costs. This aspect of pricing efficiency may be called storage efficiency.

In summary, values accruing to individuals as a result of economic activities are of vital importance in the set of values influencing the welfare of a society. Improving the productive efficiency of these activities would undoubtedly have a positive effect on realized welfare. Our present level of knowledge in the field of empirical economic research is not sufficient for accurately assessing, in a physical quantitative sense, the degree of inefficiency in actual economic operations of an industry. However, it is believed that relevant indicators of inefficient production can be devised from price interrelationships with respect to space, form, and time (storage).

\section{Specification of the Problem and a Simple Model for Analysis}

Preliminary observations and a compromise. To the casual observer, the complex activities of the manufactured dairy products industry might appear as a chaotic system. It is easy for one to develop such an impression from the multitude of products, price relationships (geographic, for one product form relative to another, and over time as the seasons pass and the general price level changes), and the intricate operations of production, distribution, and storage, which the industry manifests. The purpose of this study is to investigate whether there is or is not any justification for such a viewpoint. A rigorous approach to this problem would be based on microeconomic appraisals at each level of the marketing system, including form, spatial, and temporal aspects. Physical volumes involved in the productive processes would have to be taken specifically into account as well as the price conditions associated with such volumes. This would be an enormous task.

A cruder analysis, based essentially on price manifestations, will prove more workable and still provide a basis for comparative evaluations. Consequently, quantitative data on physical volumes will be used only in rough determinations of marginal points of physical transfer shifts to be used in the analysis of the price data. For instance, actual shipment data will be used to determine marginal points of supply for a given product. These points occur where equal returns come from shipments to demand centers located in opposite directions and are located where actual shipments tend to divide into the separate destination categories. The appraisal of the efficiency of the industry will be based largely on price interrelationships.

As already suggested, to render any judgment on efficiency requires that a valid standard be constructed to be used as the basis for comparison. In economic terms, an efficiency model must be developed to represent the operational structure of the industry. To be complete, such a model would have to include every facet of the system, but for practical reasons the detail must be reduced through simplification. Some losses occur through this process of simplification, and gains accrue through the greater clarity and manageableness of the core elements. The problem of model specification reduces to one of eliminating insignificant details without destroying the interrelationships of the major forces. 
The position to be taken in this study is one that recognizes a social environment to be dynamic and a result of social action, without a complacent acceptance of a status quo. The perfectly competitive assumptions that form the model used in this paper are not believed to have been fully admissible in past or present environments of the industry. It is believed, however, that some minor forms of social action could correct any consistent bias which the past data might show in comparison with results postulated by the model-corrective action might only involve activity in the market in light of such knowledge. Further, it is believed that whatever corrective action would be necessary to make the model feasible would not destroy other func-
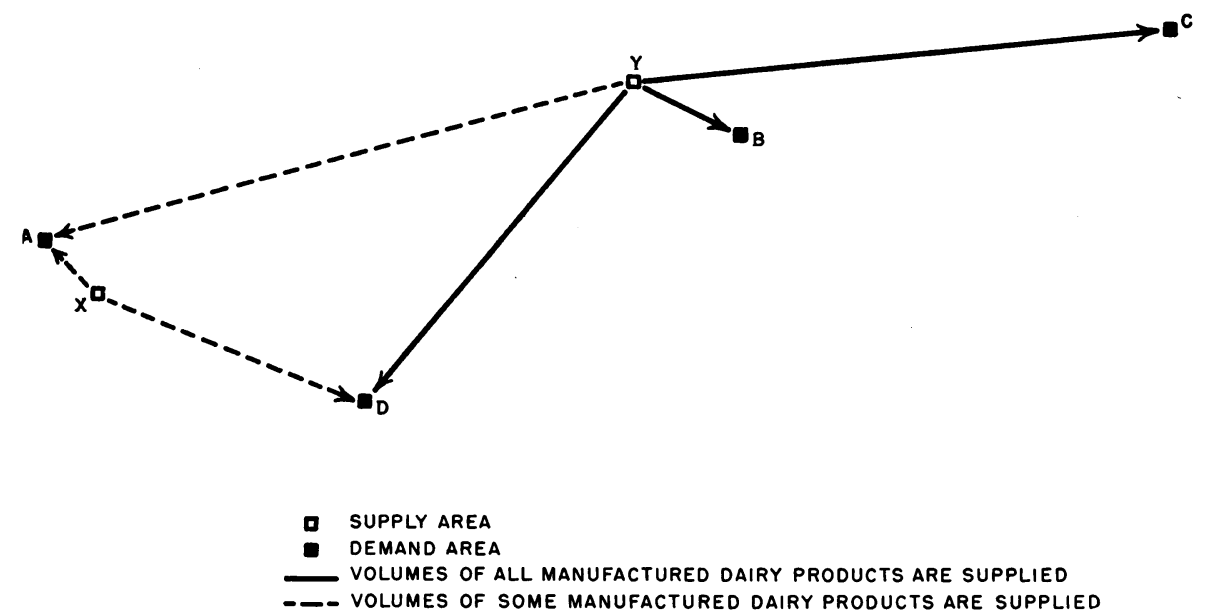

Fig. 1. Simplified illustration of supply-demand interrelationships for the manufactured dairy products industry.

tional aspects of the industry. The analyst need not design the required social action for accomplishing the change but merely present the factspermitting society to make the decision on method and action.

A price model for the manufactured dairy products industry. The following model is composed of a minimum number of elements that depict simplified situations of supply and demand for the industry through the levels of the marketing system to be considered. Although a few more hybrid situations might be envisaged, they will not be of interest for this study. Greater realism might be achieved if the elements $(A, B, C, D, X$, and $Y$ ) in the model were increased by suitable multiples, but this would result in difficulty of presentation. Figure 1 has been drawn to show only two facts, namely, geographic areas of primary supply and demand, and the general movement of products when the system is in equilibrium. In this illustration, $A, B, C$, and $D$ are major consumption points of concentrated demand while $X$ and $Y$ are small areas of concentrated supply. All fresh fluid milk requirements of the consumption points are filled by local supply areas, and any surpluses that are converted into manufactured products are assumed negligible. The fresh fluid milk supply areas are not shown on figure 1 since they are not a part of this argument. 
Since the perfectly competitive assumptions underly this model, all economic agents are atomistic in their volume operations compared with their respective aggregate by type. Consequently, there are numerous farmers and processing firms at the supply areas and numerous wholesalers at the market consumption points. Similar conditions would exist at the retail level, but that sector is not to be considered in this study except through a general recognition that the demands of all levels investigated are derived from the retail demands.

Before giving detailed discussions about particular aspects of the market structure implied by figure 1, a brief outline of the entire, interdependent system will be presented now. The brevity of this development is no indication of its importance. To comprehend and appreciate the complete set of interrelated activities is fundamental.

Underlying the economic activities of the simplified model are basic forces that determine the results. They are as follows: (1) consumer demands for final products at their locations, at specific points in time, with volumes varying according to prices; (2) costs of moving products through space and time (storage); (3) costs of converting milk into specific products; (4) physical conversion ratios between milk and products; (5) costs of producing milk on farms, giving rise to the supply function for milk at a point in time, at a delivered location, with volume varying according to price; and (6) all individuals are rational, making economic decisions by choosing the most profitable alternative. Equilibrium is reached when the interacting forces place all individuals in their best positions, subject to the particular constraints surrounding their decisions.

Visualize the chain of interactions that transmit the demands of the consumers back to the raw milk source, with each agent in the chain altering the initial demands so as to reflect the cost forces or physical conversion factors associated with his contribution in the marketing process. Each of these agents or middlemen can be placed in a specific functional category of the marketing system (retailers, wholesalers, storage operators, and processors), and their alterations of the initial consumer demands represent the particular costs that they pay to owners of services (other than the products) that are used in performing the marketing functions. These costs must be covered and a portion of the initial demands must be subtracted as the consumer demands are passed along the marketing chain in the direction of the raw milk source. If physical conversions occur at a functional level, the net demands must be converted also. Eventually, the demand forces are presented to the producers of milk in their net forms.

In a reverse direction, the supply. costs of milk are transmitted simultaneously along the chain in the direction of the consumer. Middlemen add their particular, functional costs and convert the raw milk supply function, in a physical sense, wherever physical changes are accomplished. Finally, the consumers are presented with the supply forces associated with the producer reactions to their demands.

Numerous individuals are involved in the performance of each function in the marketing system, and competitive bargaining will result in the transmittal of competitive costs throughout the system. Freedom of entry and 
exit is assumed, and the inducement of profits or the suffering of losses in a given function will lead to volume changes that will tend to force alternative possibilities to have equal and competitive advantages. Consequently, the decisions of middlemen will tend toward the production and distribution of products in absolute and relative volumes such as to equilibrate the demand and supply forces arising at opposite ends of the marketing chain during any short-run time period. In the long run, the entry or exit of consumers into the demand field, middlemen into the processing and distribution field, and of farmers into the milk supply field will lead to gains to all sets of individuals at only a competitive level. Equilibrium will have been reached.

It should be stressed that all the competitive agents in the system make decisions on buying and selling their particular volumes on the basis of a single price for each product. They are unaware of any price effect which their purchase or sale may produce. Consequently, equilibrium for the system is equivalent to a state in which the structure of prices in all its dimensions is such that all buyers and sellers are satisfied with their contracted volumes and no one is making more than competitive profits.

From the geographical point of view, one should visualize a price structure for a given product starting with the equilibrium prices in the demand centers and radiating outward along the available transportation routes through the supply areas with the level decreasing by amounts equal to the supply cost of the services involved in the movement of the product from each geographic supply point into the best alternative demand center. At each effective supply point, the equilibrium demand price is equal to the supply price, and the relevant demand center (that one with the highest net demand price) secures the volume of the product arising at that point. It is obvious that a multiple demand-supply market will produce a dissection of the total supply area into areas supplying particular demand centers. Since various products arise in the demanded transformations of manufacturing grade milk, the above remarks would apply to each and every one of them. Furthermore, because of the differences in conversion ratios and transportation costs, a further dissection of the supply areas results from the economic development of specialized areas for the production of the various products that are supplied to a given market. In general, products with high conversion ratios will be produced nearer the demand centers and those with a low conversion ratio farther away. This is illustrated later in figure 2. In that figure, the product prices have been converted to a milk equivalent basis and all processing as well as transportation costs removed.

What has been stated for the geographic price structure has suggested the relative price structure for the various forms. At the margins between the specialized supply areas, the net product prices would be such as to produce a single net value for milk equal to the supply price of that milk. It should be obvious that the equilibrium temporal variation in the price structure could be presented in a manner analogous to the geographic aspect. One need only substitute storage costs for transportation costs and time for distance. 
A more detailed discussion of these equilibrium aspects and theoretical problems arising therefrom is in order. After these details have been considered, a specific statement will be given on the consistent price interrelationships employed in this study.

What are the conditions visualized within the supply areas $X$ and $Y$ ? Without elaborating very much at this point, $X$ is a semispecialized supply area and $Y$ is a general supply area. In competition at markets $A$ and $D, Y$ has an absolute advantage over $X$ in supplying one or more of the major manufactured products. This has led $X$ to specialize in producing those products with which it can meet the competition of $Y$ in markets $A$ and $D$. One or more of the alternative primary processing operations for the raw milk has been economically squeezed out of $X$ although the major products of such operations might still be produced as forced by-products of one of the remaining operations. Assuming that processing costs at $X$ and $Y$ do not differ greatly, the primary cause of the economic elimination of one or more alternatives at $X$ is the higher opportunity cost associated with the employment of resources in the farm production of manufacturing grade milk at $X$ than at $Y$.

Limiting the discussion to area $Y$, from the farmer's viewpoint there are three major alternative outlets for his milk, namely: (1) use on the farm; (2) sale to fluid use plants for local consumption; and (3) sale to manufactured dairy products plants. At any point in time, certain institutional barriers might prevent a farmer from shifting to alternative (2), but no impediments are assumed to exist for the subalternatives of (3). As a going activity, farmers within area $Y$ will be producing a certain total flow of milk, $Q$, and will have established allocations $Q_{1}, Q_{2}$, and $Q_{3}$ to the three major outlets. Whether these allocations conform to a competitive equilibrium for the whole dairy industry is not of interest here. What is of interest is whether the suballocations of $Q_{3}$ are in equilibrium. The answer to this question will be based primarily on price interrelationships instead of the actual volumes.

The subalternatives of (3) within $Y$ will now be specified with the listing signifying the major product for each type of plant operation. These operations are for whole milk utilization and are as follows: $(a)$ butter plants; (b) evaporated milk plants; (c) American cheese plants; and $(d)$ whole milk powder plants.

In some of these operations, cream or skim milk will result as an initial by-product when the whole milk is standardized for the production of the major product. This cream or skim milk might be processed into numerous by-products, especially the skim milk. For instance, the latter might be returned to the farmer, used for cottage cheese, dry skim milk powder, condensed skim milk, or some other products that are less common. Cream of the manufacturing grade visualized here might be used for butter and either dry or condensed buttermilk, or as an ingredient in a formula for ice cream. Whenever cream or skim milk occurs as an initial by-product, it will be assumed that all the possible products into which each could be processed will be in equilibrium valuewise.

The term in equilibrium valuewise means that the prices and processing 
costs of the products which can be made from a unit of skim milk (unit of cream) are such that, taking individually the alternative uses of the skim milk (cream), the net value of the unit of skim milk (unit of cream) is equal in each use. This net value in a given use is the difference between gross value of the product made from the unit of skim milk (unit of cream) and the processing cost incurred in its production. With this assumption, one use of the cream (butter and dry buttermilk) and one use of the skim milk (dry skim milk powder) can be employed in whole milk utilization comparisons.

Although $(a),(b),(c)$, and $(d)$ listed above may be only a subset of the possible subalternatives of (3) in the real world, they do represent a very large percentage of milk usage in the manufactured dairy products industry of the United States. Even if those uses which have been listed were only a part of the total, the internal analysis of this subset would still be a valid and worth-while project. The introduction of one or more other uses would not affect the internal relationships in the subset considered. It would be equivalent to adding another independent operation having only one new major product. The procedure employed in this study could be expanded to cover the more complete case.

The simplified conditions surrounding the economic agents of the supply area $Y$ have been described. A similar environment exists at $X$, except for the reduced number of economically feasible primary processing alternatives. At the market points $A, B, C$, and $D$, it is assumed that competitive buying conditions exist for all products at the wholesale level. Rational buying and selling action in response to the profit motive prevails for all the agents, with adequate market knowledge existing.

As stated previously, all underlying supply and demand data for inputs and outputs would be required in specifying the complete model. Instead of becoming entangled in all of these physical volume aspects of the model, it will be assumed that the supply-demand relationships are of such nature that equilibrium can be reached with respect to the volume aspects and that specific price conditions will be indicative of this situation. Certain general (and convenient) assumptions must be made with respect to costs incurred by firms involved in spatial, temporal, and form transformations of the raw material. Consequently, the price model cannot be divorced completely from the volume aspects, and assumptions will be made about the manner in which costs vary with firm and industry output. Figure 2 shows a cross section of the price structure of figure 1 at a given point in time. The upper portion of this figure has been drawn as if all the geographic points were colinear, equal processing costs existed for each type of operation, and transportation charges were linear functions of distance. For simplicity, it has also been assumed that all milk is alike and of such composition that no standardization is required in the production of evaporated milk. Skim products of the butter operation are assumed to have zero net value. Only the two operations, butter and evaporated milk, exist. This simplifies the presentation. The lower portion of this figure is a magnification of conditions within supply point $Y$.

The particular equilibrium situation shown in the upper part of figure 2 indicates that the two operations have equal advantage at point $Y$ but that 


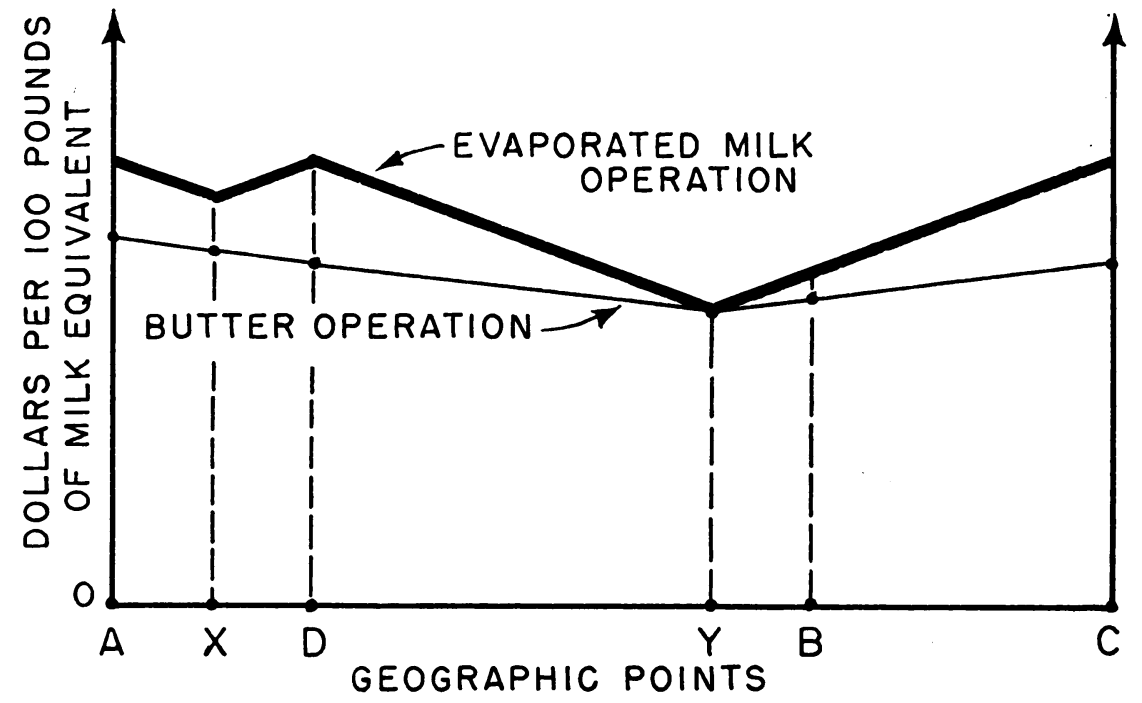

MAGNIFIED SECTION WITHIN POINT Y

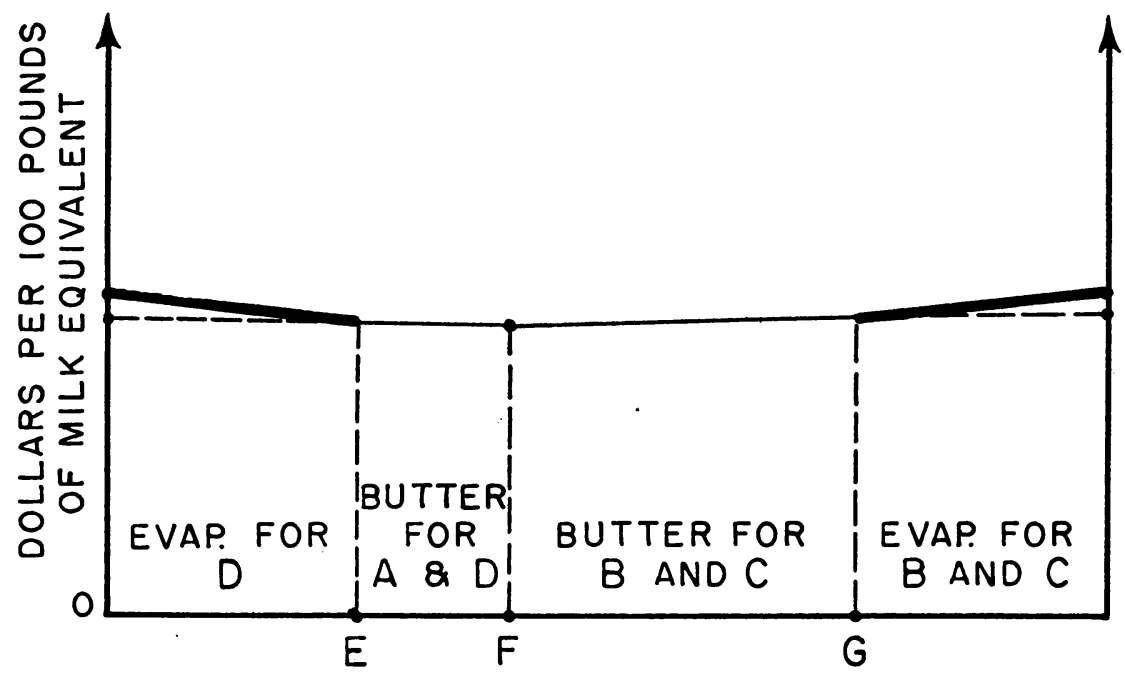

Fig. 2. Equilibrium net values of milk in two uses with geographic variations. 
the butter operation has been excluded from point $X$. Consequently, all butter is supplied by $Y$, both $X$ and $Y$ compete in supplying evaporated milk to market $D$, all evaporated milk for $A$ is supplied by $X$, and all evaporated milk for $B$ and $C$ is supplied by $Y$. Other equilibrium cases of various degrees of specialization and diversification for $X$ and $Y$ could exist in lieu of the particular case selected, depending on the supply of milk at production points relative to product demands.

One of the simplifying assumptions required to develop equilibrium conditions for the relative prices of the products is the isolocation of the four operations previously listed. This assumption, if actually true, would make for great instability in the geographic price structure over time if the building or liquidation of each type of processing facility could be achieved instantaneously at constant cost in response to sudden shifts in product demands. In reality, point $Y$ must have some area dimension and it is this fact which gives stability to the prices at all geographic points. The lower part of figure 2 indicates the allocation of particular supply areas within $Y$ in response to the transportation costs. If all four operations occurred in $Y$, a multiplicity of internal division points would exist, showing various points where paired operations would have equal advantage-such as points $E$ and $G$. However, with $Y$ small in its linear dimensions compared with distances to the consumption points, the differences in the advantages of the various operations within $Y$ would be exceedingly small. For this reason, the isolocation assumption, postulating equal economic advantage for all operations, is closely approximated over the small supply area.

It will be assumed that a sufficient amount of excess capacity inherently exists for each type of operation to permit sizable short-run shifts in utilization in response to changing demands. This assumption seems not unrealistic in view of recent changes in milk utilization in the United States. Further, it will be assumed that such increased (decreased) utilization can be achieved through a lengthening (shortening) of the daily duration interval of operation with practically no change in the rate, and that in consequence, expansion (contraction) can be achieved at approximately constant unit cost. In other words, it will be assumed that increases (decreases) in supply costs of variable inputs will be compensated by a decrease (increase) in the fixed cost allocation rate during short-run situations. One is placed in an unusual position by this statement. It is recognized that fixed costs have no place in short-run decisions and that only over many successive short-run periods need they be covered. However, to give rigor to the relative price analysis, these fixed costs will be assumed to be allocated continuously to each shortrun period and will be treated as though they should be covered by the short-run supply price of output. If long periods of persistent positive or negative interproduct price bias is observed, then inefficient utilization is indicated.

These statements refer to all costs other than for the milk. The cost for raw milk used in an operation that has experienced a relative inerease in the demand for its output over those of other operations is the opportunity net value of milk in the next best alternative. Assuming that the short-run supply of milk for manufacturing purposes is rather fixed in total as it is, 
the supply cost of milk in a given use will increase for firms rather rapidly as their aggregate use decreases the amount available for alternative utilizations. It is this rising supply cost that is the brake on the expansion of output from the favored operation, that is, equilibrium is again reached through the dual forces of lowered value per unit with increased output of the favored product and increasing cost of the milk due to increasing values per unit of the other products as their outputs are reduced. It is evident that long-run adjustments in plant numbers will be required whenever full short-run adjustments are insufficient to reach a new equilibrium. These adjustments will also be assumed to take place at constant cost. A change in the duration interval of operation can be a long-run adjustment. How a full consideration of this aspect of production would affect the orthodox concepts of short and long run is uncertain. Apparently, this characteristic meshes the two concepts into one so that no unique separation can be made.

It should be stressed in this theoretical discussion of the volume aspects of the model that the geographic segregation shown in the lower portion of figure 2 is extremely vital. It is this fact that permits one to postulate how a stable equilibrium can be reached (in terms of utilizations and geographic allocations of products to consumption points) from all or nothing reactions by individual firms. This geographic dispersion allows the dividing lines to fully separate the firms in such fashion that no firm falls at a point of indifference. If a number of firms were placed in positions of indifference at equilibrium and a concordant decision by them would not have negligible price effects, the system would be potentially unstable and not necessarily convergent upon displacement. These remarks have reference to a perfect knowledge static model that is postulated for explaining essentially dynamic activity. It appears that the perfect knowledge conditions cannot be made sufficiently complete to eliminate instability if one insists that competitive firms assume their actions have no effect on the basic data (prices) with which they arrive at decisions. The latter assumption is inconsistent with a stable equilibrium definition which places one or more firms in a position of indifference on some part of its full decision.

The point at issue in the last paragraph is fully exposed when one attempts to postulate a perfectly competitive theory of storage. Within the limits of a certainty model, it is impossible for one to specify the content of a perfect knowledge state for each firm. Figure 3 illustrates a temporally static situation in which a homogeneous, storable product has an invariant seasonal pattern of production. Consider the case of one competitive supply point and one competitive consumption point. With a stable demand, the seasonal price variation would be as indicated in the central portion if storage were not undertaken. In any real case, the exact form would depend on the production pattern and shape of the demand schedule. Assuming that storage services per unit of product per unit of time could be supplied at constant price, the price variation over the season would be as indicated in the lowest portion. The problem is to explain how this latter variation would materialize from decisions reached by competitive firms. This cannot be done, within the limits of a certainty model, for reasons developed in the discussion of figure 3 , below. 

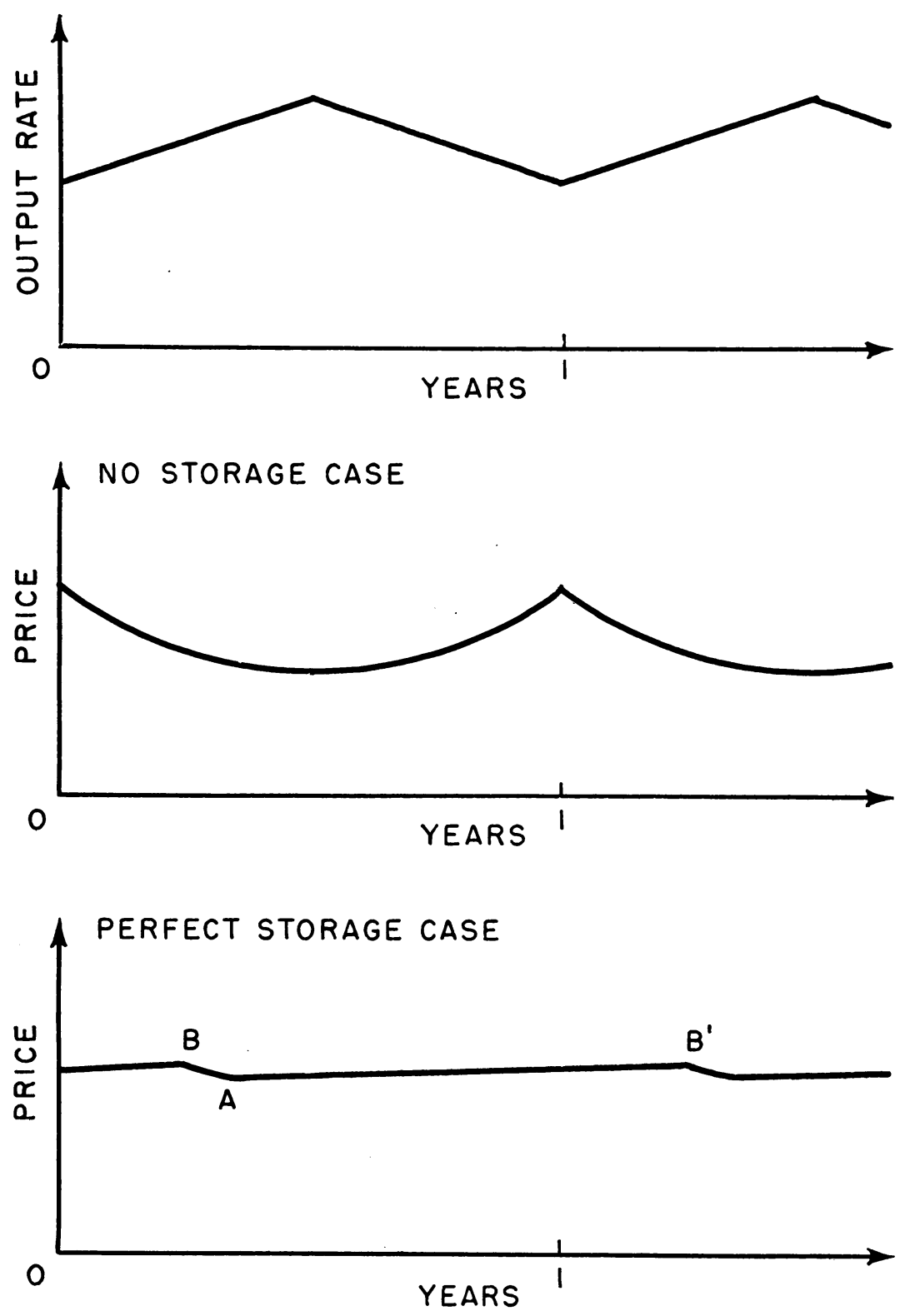

Fig. 3. Price variation for a product having a seasonal pattern of production.

Assume the industry is at that point in time when price is at $O B$ in the lower portion of figure 3 and that each entrepreneur knows the seasonal price variation would be as shown, $B A B^{\prime}$, if the aggregate decisions by all firms are of a special pattern. Between $B$ and $A$, all firms know storage should not be undertaken. At point $A$, every firm is simultaneously placed 
in a position of indifference between current sale and storage. Progressing from $A$ to $B$ through time, this situation of indifference must continue or the indicated price variation will not result. It is difficult to visualize what element of perfect knowledge would enable each and every firm (acting individually) to choose between sale and storage through time in such fashion that the aggregate effect would be the equilibrium price variation. Should individual decisions on sale or storage be a matter of chance in a situation of indifference, an aggregate result of violent instability would be as likely to occur as the equilibrium movement.

Recognizing that potential inconsistencies and logical instabilities are inherent in certain phases of the perfect knowledge competitive model, it probably would be more reasonable to introduce uncertainty, differing storage costs, and diverse expectations into the explanatory system. In the real environment, the existence of various expectations by entrepreneurs about the values of future data upon which current decisions are based and the continuous revision of these expectations in the dynamic setting temper the violence of adjustments. Through time, these imperfections tend to make for a self-correcting system. These considerations would be particularly helpful in explaining how individual reactions could approach perfect storage operations. It would indicate which firms would sell currently, which would store, and which few, if any, would be indifferent at each point in time. However, the present knowledge of this approach is not well developed, and the form of the frequency distribution of expectations has not been defined through deductive processes.

Having discussed sufficiently the problems involved in explaining how an industry equilibrium is reached with respect to form, space, and time aspects of production and distribution, the price conditions which would be functionally consistent with such a state will now be given in detail for a simplified version of the manufactured dairy products industry. The isolocation assumption for firms at supply and demand points will be employed and the instability consequences will be ignored.

The consistent price relationships. The equilibrium allocations of milk to processing plants and products to consumption points will result in the following price conditions:

1. At plants in all locations comparable with $X$ or $Y$, a single supply price equal to a single demand price for milk of a given fat content will exist. Since milk of various fat tests will be produced, the extension of the condition in the last sentence gives a single price schedule over the fat test range. This condition will prevail individually within $X$ and within $Y$, with the two price schedules not necessarily being equal. Reverting again to a consideration of milk of a given fat content and using the hundredweight as the milk unit, the single price stated above will be equal to a common net value to all plants resulting from the sale of the products yielded by 100 pounds of milk. The net value of a hundredweight of milk at any given type of plant is the sum of the product weights times their respective net prices, from which is subtracted any general costs than can be rationally allocated directly to the handling of the milk. The net price of a specific product is the f.o.b. plant price minus the supply cost of the services uniquely involved in the processing 
of the product, per unit of weight. A single supply cost for the services used in processing a milk component into a given product will prevail for all plants, irrespective of whether the product is major or merely a by-product. Also, a single f.o.b. plant price will exist for each product at all plants producing the product at this location. The last two conditions produce equal net prices for each product at all plants producing the product at this location. Again, to avoid confusion to the reader, it must be stated that all equality statements made in this paragraph will hold specifically only within $X$ and within $Y$ and not necessarily for both. There is no reason why the net prices or the supply cost of services should be equal respectively at both $X$ and $Y$. Such equality could exist but is unnecessarily special.

It should be noted that an arbitrary, but useful, breakdown of the total processing cost incurred by a plant is implied in the above statements. For instance, it has been assumed that one could allocate costs in a butter-dry skim milk operation into three categories, namely: (1) all prehandling of the whole milk up to the point where the cream goes into the churning process and the skim milk into the drying process ; $(2)$ butter making and packaging ; and (3) skim milk drying and packaging. Further, it will be assumed that (1) is equal for all plants per 100 pounds of milk, and that costs like (2) or (3) will be equal regardless of the type of compound operation in which the specific process occurs. Because of certain joint costs involved, an empirical breakdown would be difficult. However, such joint cost problems appear to be relatively small and so the assumptions seem reasonable. In the development of the relative price analysis, these cost assumptions are almost indispensable.

2. Singular wholesale buying prices will exist at each consumption point for each product. Spatially, an equivalent price condition will hold for each product whether at the consumption points or at the supply points. Specifically, the prices for a unit of a given product at all those market points that receive this product from a common supply point (either a partial or full supply) will differ only by the differences in transportation and handling costs per unit of the product from this common supply point to each of the markets. In figure 1 , this would hold for all products at $B, C$, and $D$ based on $Y$, and at $A, B, C$, and $D$ for all those products that are included in movements from $Y$ to $A$. A similar statement could be made for markets $A$ and $D$ based on $X$ but would cover only a reduced list of products. An inverse statement can be made for the spatial relationship of f.o.b. plant prices for products at different supply points. The f.o.b. plant prices per unit of a given product at all those supply points that jointly supply the product to a common market point will differ only by the differences in transportation and handling costs per unit of the product to this common market point from each supply point. Figure 1 indicates that this would hold at $X$ and $Y$ for those products jointly supplied to $A$ and perhaps separately for those products jointly supplied to $D$ if they are not common in both cases.

3. The nonperishability of the products permits competitive gains to be realized through the operation of storage. Numerous dynamic changes could motivate storage activity. These changes can be separated into two cate- 
gories, namely, those influencing supply and those influencing demand. In the real environment, there is little justification for assuming static conditions for either supply or demand. On the supply side, there is an almost predictable seasonal variation in raw milk production, which usually overshadows both the relative and aggregate changes in the demands for products. The relative net prices of the products will definitely influence the manner in which this seasonally varying milk supply alters the production rate of the various products. Since the effective supply of a product is the current production plus the net change in storage stocks and it is this supply reacting with the demand which determines the price, it is easy to see that the storage operations have a great effect on the composition of products (both at points within the season and in total for the season) as utilization shifts keep relative net prices in equilibrium at all time points in the season.

Before specifying what the temporal pattern of prices over a season would be if storage operations were efficient, a few more aspects of the problem should be considered. Storage costs for the various products are significantly different. Neglecting the secondary by-products, 100 pounds of milk will yield approximately either 5 pounds of butter, or 10 pounds of cheese, or 12 pounds of dry whole milk, or 1 case (48 cans, each weighing $141 / 2$ ounces) of evaporated milk. Assuming the f.o.b. plant relative net prices of these products at $Y$ are kept in equilibrium by utilization shifts of the raw milk irrespective of the price level, under conditions of stable demands, only that product with the smallest storage cost per unit of time for the amount of that product which 100 pounds of milk will yield would be stored. With relative net prices held in equilibrium, the amount of price variation of the other products in response to the price variation of that product whose storage cost per 100 pounds of milk equivalent is least would be insufficient to cover their storage costs.

These remarks are based on two assumptions. First, the price variation of the product with least storage cost per unit of milk equivalent, if storage were not undertaken, would be greater than the storage cost per unit of the product between the time points of maximum and minimum price. Otherwise, no product would be stored. Second, the demand for the product having least storage cost per unit of milk equivalent must be of sufficient magnitude so that the shift in milk to that use, upon the initiation of storage in the seasonal cycle, can be absorbed when withdrawals from storage occur at later points in the season.

Should the build-up of storage stocks make it impossible at later times to maintain a price variation continuously consistent with the storage cost (as is shown in the lowest parts of figure 3), while relative net prices were held in equilibrium, then a very complex seasonal variation in the product prices (and also the milk price) might result. More than one product might be stored and that one with least storage cost per unit of milk equivalent would have storage begun and ended at an early interval of the season and withdrawal from storage at a disjoint interval later in the season. Depending on storage costs, a second product might have a period of storage and withdrawal between these two time intervals. Since this case is not believed to have been the situation for the manufactured dairy products industry, 
it will not be investigated further. Butter has been a very dominant use for manufacturing grade milk, and it appears to have had the least storage cost per unit of milk equivalent. Consequently, the price variation shown in the lowest portion of figure 3 is appropriate.

Unless the utilization of milk for producing a product that would be stored was very dominant, a large seasonal variation in the daily rate of producing it would occur. The other products would probably be more stable in their daily rates of production, although this would depend on their individual price-quantity relationships. Intraseasonally, the unit processing cost of the economically storable product would probably vary more than similar costs for the other products with a low during the flush period and a high in the deficit period of milk production. If firms react to current cost rates instead of the seasonal average rate, this condition alone would reduce the amount of seasonal variation in the net price of the stored product as the full price varied sufficiently to cover storage cost. The cost variation postulated for the processing of the stored product is not assumed to be caused by the allocation of fixed costs at different rates to the units of the product produced at different periods within the season. An equal rate to all units for the whole season may be more rational for such allocation. Rather, the increase in processing cost in the deficit period is assumed to be a variable cost rate increase caused by the plant's being forced to operate at an inefficient rate due to a low volume of milk receipts in conjunction with a partially inflexible length of working day. With processing costs of other products constant over the season and with relative net prices held in equilibrium, the full price of the other products would vary less than the amount caused by storage cost of the stored product. This fact would place the other products at a further disadvantage with respect to storage.

The variation of product prices over the season, assuming a perfect storage pattern, will be as follows. For those products that are stored, the price variation will be such as to cover the full storage cost of the volumes that are in storage at each point within the season and sold at later points within the season. For those major products for which storage is economically infeasible, the price variation will be keyed to the price variation of the stored products in such a way as to produce equilibrium relative net prices-the latter being those relative net prices of the major products that result in equal net values for 100 pounds of milk of a given fat test in all uses.

For this discussion, it will be assumed that high or low fat test milk has no particular advantage in a given use relative to the other uses so that the equilibrium relative net prices of the major products can be expressed as functions of the net prices of the by-products relative to the net price of one (the base) of the major products. Insofar as by-product demands and individual storage potentialities have no fixed connection to similar properties for the major products, the net prices of the by-products hold no constant relationship to the net price of a major product. This means that the price of manufacturing grade milk at a point like $Y$ will vary seasonally under equilibrium relative net prices for the major products in a manner not completely determined by the variation in the net price of the major product that has least storage cost per unit of milk equivalent. Although the latter 
will have a very dominant effect, the influence of the movements of the net prices of the by-products relative to the base product must be taken into account when postulating the seasonal variation in the price of milk. Only those by-products which are not major products in some other use are involved. This limits the implications to the possible by-products of skim milk, buttermilk, and whey, or in this paper, to dry skim milk, dry buttermilk, and dry whey.

This completes the statement of price relationships that would be consistent with a continuous state of equilibrium for the industry. In the real world of uncertainty and time-consuming adjustments, deviations from these equilibrium price relationships are to be expected. In fact, deviations of rather large magnitude might be required in order to motivate adjustment activity. However, consistent bias in these deviations (always positive or always negative) is not to be expected if the industry functions efficiently, unless the dynamic changes causing the bias are of such unidirectional nature that the adjustment process never succeeds in removing the discrepancies of advantage and/or disadvantage. Recent trends suggest that this has not been the case.

Some difficulties in the application of the model. Before proceeding to the empirical analyses, a few words should be said about the general difficulties involved in going from an abstract model to the actual activities. In the first place, and as has been suggested, some question may be raised regarding the propriety of the type of model that has been outlined. The proposed model is of the static type and the actual activities are essentially dynamic. Static models avoid the temporal paths and explain adjustments in a time void. Because of this neglect of the temporal paths, these models can only consider flow rates ( velocities) and cannot cope with rates of change in flow rates over time (accelerations). The acceleration interdependencies are probably more important than velocity characteristics in the evolution of economic growth and change. It is probably true that the static model is a good approximation to the dynamic system when rapid adjustments without significant lags are possible in the economic activity under consideration. Economists have not progressed very far up to the present in the development of dynamic models, so static procedures are still the principal analytical tools.

Special troubles arise in the money valuations of inputs and outputs. Certain inputs have no evident market for price determination, for instance, inputs from durable equipment. Margins for future uncertainties cannot be accurately valued. Quoted prices for outputs are not always effective prices when discounts or premiums are involved. Frequently, one is forced to employ prices that are for broad areas (space or time) when point values are implied by the model. These and many other price data problems occur in the transition from model to reality. They will be considered specifically as they are encountered in sections that follow. Further, in view of the fact that the writer does not know that the competitive model used for comparative purposes in this study is a valid welfare model, all efficiency statements on pricing results should be interpreted as conditional. With this understanding, the adjective conditional will be dropped from such statements and alluding discussions. 


\section{Analysis of the Geographic Efficiency of the Manufactured Dairy Products Price Structure}

In the preceding section under the second condition of the statement on equilibrium price results, the spatial characteristics of the price structure of the products were specified. Actually, a dual specification was made at that point-one with respect to prices at the wholesale-buying level of the different consumption centers and the other with respect to prices at the various joint supply areas. Viewing the actual values of the prices of a given product (for the transfer, plant to first buyer) at all the effective geographic points as elevation measurements on a price terrain, the first specification would be analogous to a comparison of the peaks and the second to a comparison of valley levels. The rise from valleys to peaks would be the transportation and handling cost gradients.

The analysis that follows will cover only three products: butter, American cheese, and evaporated milk. Data are not available to expand this list to include those other products making up the manufactured dairy products category. Comparisons of prices for butter and cheese will directly utilize wholesale prices at a few major cities in the United States (peaks), while the evaporated milk prices which will be used are approximately equivalent to f.o.b. plant prices (valleys). Unfortunately, the latter prices are Geographic Division averages, and data on the distribution of evaporated milk from these artificial supply areas to consumption points are not available.

The geographic price norms. Basically, the second equilibrium condition specified in the preceding section is the norm for the analysis of the efficiency of the spatial price structure for the products of the industry, that is: $(a)$ wholesale buying prices for a given product at all points supplied by a common point will differ from one another by their differences in transportation and handling costs from the common point of supply, and (b) prices f.o.b. plant for a given product at all those points of supply for a common consumption point will differ from one another by their differences in transportation and handling costs to the common consumption point. All prices and costs are on a unit of weight basis for each product. Under the abstract assumptions of costless, instantaneous shifts of timeless product movements, and of adequate knowledge of prices, sellers would efficiently allocate their supply volumes to minute price advantages. A similar argument could be given from the buyer's point of view. Within such a frictionless environment, conditions $(a)$ and $(b)$ above would hold with practically no discrepancies over time.

Reasons for deviation of actual prices from the norms. Such a utopian condition fails to exist in reality. Rather large biases from geographic price equilibrium are to be expected. After several months of continuous bias in market prices, volume movements should be expected to change in response to the advantages and disadvantages of the distortion. However, the cost involved in securing new buyers for the marginal sellers and new sellers for the marginal buyers might deter such volume movements in response to price biases. This is especially true if the dynamic causes for the short-term price distortion are somewhat random and, after a rather short-time period, 
either rectify themselves or perhaps even reverse the direction of the bias.

When the analysis of the geographic price structure for a given product is made for a time period of many years, important changes are likely to occur in the shape of the price terrain. This is particularly true if the supply areas are not economically fixed points but are quite extensive geographic areas. The position of the marginal supply valleys, the shape of the transportation gradients, and the level of the consumption point peaks are not invariant over time. Transportation rate changes may be either absolute or proportionate and not necessarily uniformly incident on all supply areas. Over longer periods these changes influence the location and intensity of production, causing shifts in the position of marginal supply areas. Additional changes in opportunity costs at the various supply areas will make for similar shifts. On the demand side, forces affecting demand intensities (population, tastes, disposable incomes relative to available consumption goods, et cetera) will vary in their influences at the various consumption points, producing further shifting of marginal supply areas. In summary, dynamic changes in conditions of supply and demand will cause the price structure for a product to vary geographically over time.

An arithmetical approach. The procedural problem resolves to a recognition of the dynamic changes in supply and demand on the location of marginal supply areas for each product. No particular emphasis will be placed on rationalizing supply area production and location. This will be covered in the following section on relative prices. In general, an arithmetical approach will be used in lieu of statistical inference tests (regression, rank, or homogeneity) for the spatial analyses. There are two reasons for this decision. First, the effects of the dynamic changes cited above cannot easily be eliminated from the price data in order to arrive at values that rationally could be assumed to have been drawn from a stable population. Since the quantitative values of these effects are not known precisely, a more liberal qualitative rationalization will have to be given. Second, even if these effects could be quantitatively eliminated (or the test revised to include them), a problem would arise when interpretation of the test results was made explaining the statistical results in terms of the economic environment.

Preliminary evidence of geographic price consistency. Before beginning the specific product analyses, preliminary evidence shown in figure 4 should be considered. Actual prices for evaporated milk, butter, and cheese, for three markets in each case, have been plotted for the months from 1921 through 1950. These absolute prices show large variation in levels over this time period and a remarkable consistency in the movements at the designated markets. In general, prices for a given product at the various markets follow each other very closely through time. Inclusion of more markets would not have changed the general picture. This figure suggests that geographic price relationships have been highly consistent, but a more careful evaluation might discount this fact, since this information does not take into account the changing locations of supply sources and changing transportation costs. Consequently, the following investigations will focus on the small differences between these absolute prices at various markets in conjunction with locations of marginal supply areas and transportation rate changes. 


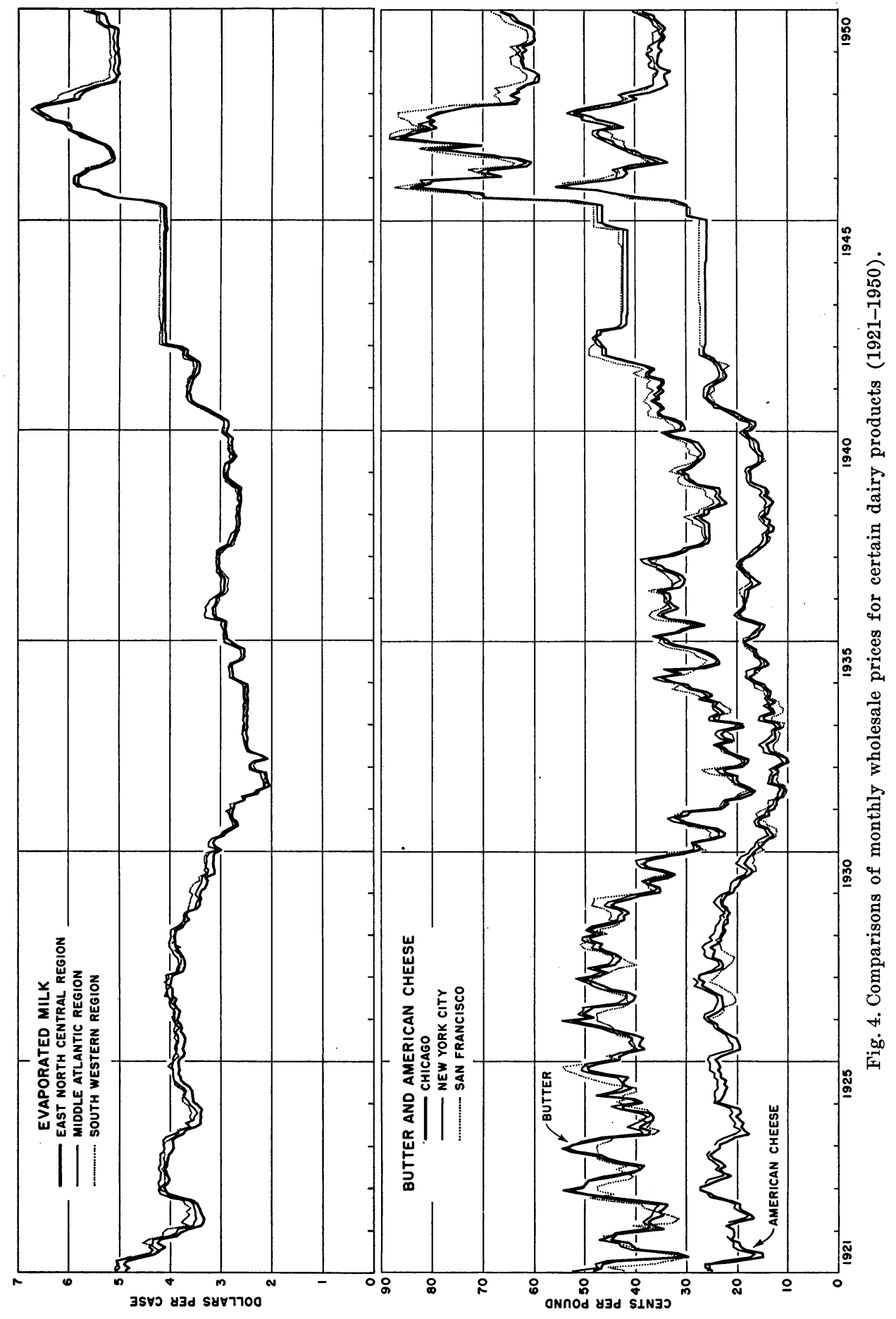




\section{A. Analysis of the Geographic Structure of Butter Prices}

The primary data consist of monthly average wholesale prices per pound of 92-score butter at Chicago, New York, Boston, Philadelphia, San Franciseo, and Los Angeles for the period from 1921 through 1950. Some of these price series fail to cover the entire period. Throughout all of this period the Chicago, Boston, New York, and Philadelphia markets secured their marginal supplies in the western parts of the concentrated butter-producing area of the Midwest. Since the transportation rate schedule from these midwest points to eastern destinations increased at a decreasing rate as a function

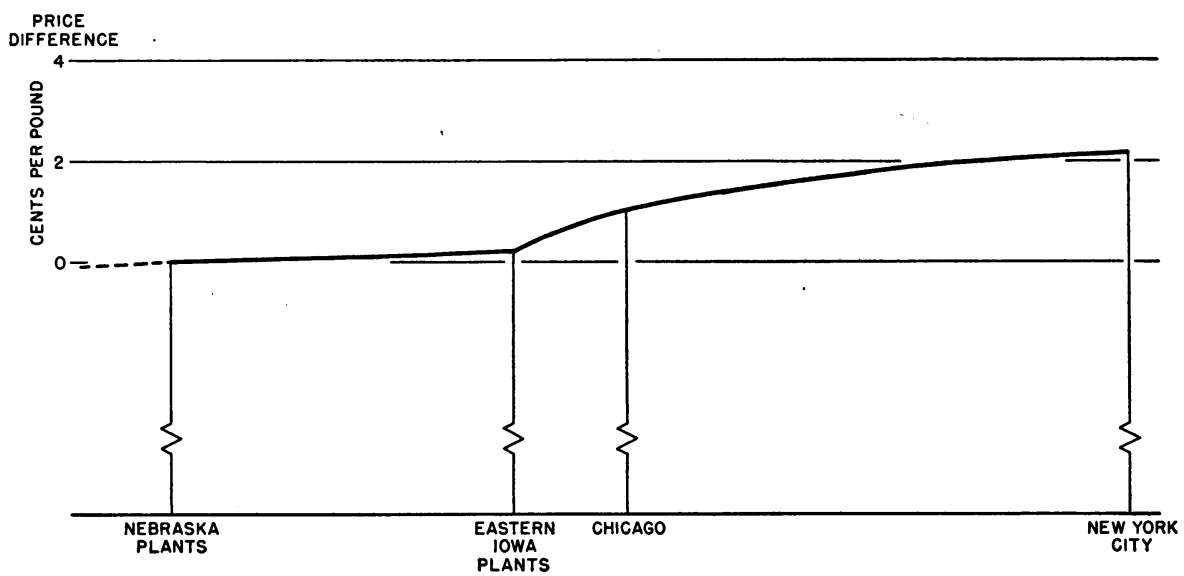

Fig. 5. Illustration of a butter price profile, Nebraska to New York City.

of distance, the more westerly parts of the supply area found that the more distant markets to the east were more advantageous outlets than Chicago and other less distant population centers. Further, because of the rather flat zonal rate structure for shipments from these westerly points to the eastern seaboard, the price profile probably looked somewhat like figure 5.

Prior to the mid-1930's, the Mountain and Pacific region was probably on about an aggregate balance between consumption and supply with a partial deficit condition in California being filled by local excesses primarily from Idaho and Oregon. Later, the California markets also had to reach into midwest areas so that the entire United States market became a highly integrated unit. Since World War II, the rather marked expansion of butter production in Idaho has tended to counter the need for marginal supplies from the Midwest even in the face of a rapidly increasing Pacific Coast population and income situation. In spite of this, a phenomenal increase in the receipts of butter at Los Angeles from Missouri has been observed. This latter aspect might be the result of round-trip trucking operations between the two areas, which have grown during the postwar years. The westward extension of the butter-price profile since the 1930's would probably have a pattern similar to figure 5, except that it would be reversed from right to left.

Assumptions. With this short descriptive background for butter movements from plants to consuming centers, a more formalized statement about 
the assumptions underlying the analysis can be made. First of all, the nature of the wholesale butter prices collected by the United States Department of Agriculture Market News Service must be clarified. These prices do not represent prices paid by receivers of butter from producing plants f.o.b. the city location of the receiver. Rather, they are one step farther along the marketing channel to the ultimate consumer, that is, they are selling prices by the receiver (or any other possessor of wholesale lots of butter) to the trade which purchases wholesale lots of butter. Since prices paid by receivers are desired, some assumption must be made about the receiver's margin in order to be able to use the United States Department of Agriculture quotations. The assumption is that this margin is equal for all receivers at all major cities for a given month. Minor differences in this margin would probably be insignificant relative to the actual price differences of the other markets over Chicago. With this equal margin assumption, differences between the United States Department of Agriculture prices for a given month at the various markets would represent actual differences in the buying prices of receivers of butter at these markets. For convenience of procedure, the handling costs involved in unloading and temporary storage of the butter received from the plants will be assumed to be included in this margin.

Next, it must be admitted that the available data on destinations of shipments by plants and associated transportation charges are scant. Some statistics are available on volume of receipts and state of origin for shipments to a limited set of cities. Normally, more localized origins would be required in order to delineate theoretical supply areas for the various markets. However, if seasonal changes in production or random supply-demand movements produce a continually shifting set of theoretical supply area boundaries, it is likely that actual shipment data would show a large amount of interspersal of origins over rather wide geographic areas. As was stated previously, the cost of frequently initiating and breaking buyer-seller contracts would substantiate such a condition. It is believed that the existence of transportation costs (the basis for delineation of theoretical supply areas) has a strong influence on purchase and shipment decisions and that gradually supply areas and prices will show the reaction of buyers and sellers to this constraint.

What can be done to eliminate the lack of clarity exhibited by the data on market supply areas? Seemingly, it would be difficult to explain the outshipments from origins in butter-deficit states. Frequently these outshipments were in small volumes, and a rational explanation might be given if the particular circumstances were known. These semi-irrational shipments will be overlooked in the process of attempting to fix the actual supply areas of the past. Whenever the data show insistent new trends in origin of shipments, such information is taken as evidence of a shift in intermarket supply area boundaries and can be used to fix approximate previous locations.

Supply area boundaries. Assuming that the three eastern markets (Boston, New York, and Philadelphia) have a common midwestern supply area for marginal supplies, there are two major intermarket boundaries to be fixed. One boundary is between Chicago (and nearby population centers to the east) and the three eastern markets. The other is between the western 
markets (Los Angeles and San Francisco) and the eastern markets. Remembering the rather flat zonal nature of the transportation rates from the plains states to either coast, errors in positioning boundaries in that area will not prove to be very significant. They will have a very minor effect on net price comparisons.

Tables 1 and 2 have been computed to assist in fixing these boundaries. The information in the first of these two tables suggests that the boundary between the Chicago market and the three eastern markets could be approximated, as shown on figure 6, for the entire period, 1921-1950. Mason City,
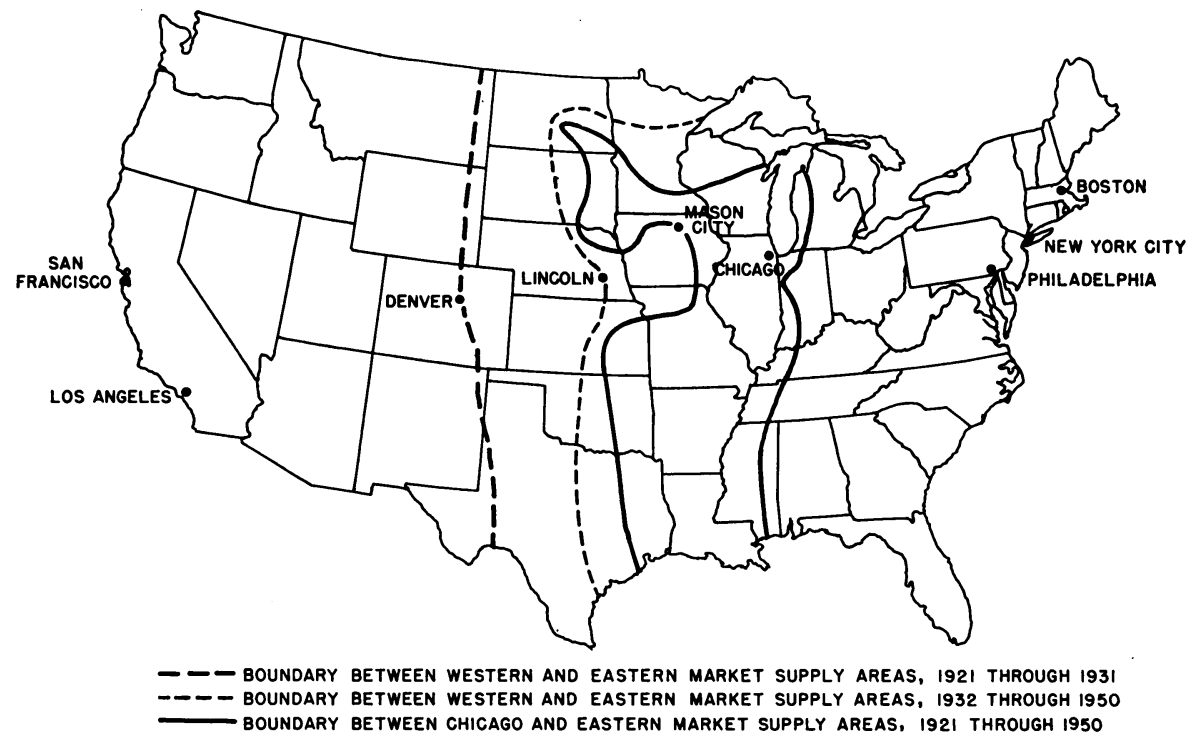

Fig. 6. Boundaries between major market supply areas for butter in the United States (1921-1950).

Iowa, will be chosen as a representative origin on this boundary and will be employed in estimating expected price differences for the eastern markets over the Chicago price. The section of table 2 that considers eastward shipments by the Mountain and Pacific states seems to indicate that the shipments from Colorado have, in general, declined since about 1930 (excepting 1933, 1937, and 1938) and that the remaining shipments could be classified as semi-irrational, especially since the preceding date. High volumes in 1944 and 1946 from origins excluding Colorado were probably caused by movements of government stocks. In any event, total shipments from western states were insignificant compared with total receipts at the four markets.

The above conclusions about the status of Colorado, coupled with the data given by the other part of table 2 showing an insistent rise, since 1931, in volume of receipts at Los Angeles and San Francisco from states other than Mountain and Pacific states, indicate that prior to 1932 the easterly limit of the seasonally shifting boundary between eastern and western market supply areas probably ran in a north-south line through eastern Colorado. Denver, Colorado, will be selected as a representative origin on this bound- 


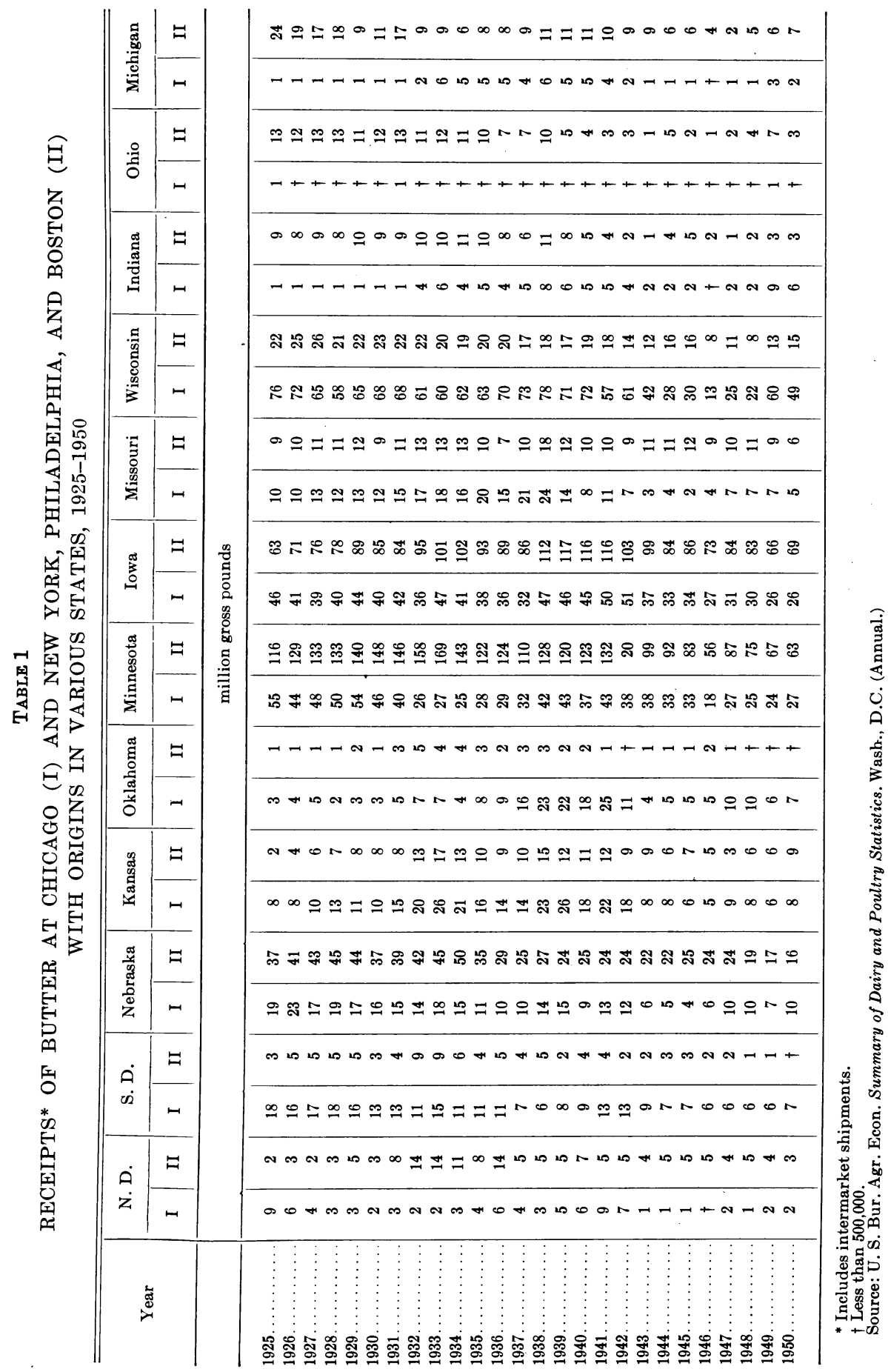


ary. Since 1932 (the data in table 2 probably would place this shift about 1933 or 1934, when the evidence is stronger; the year 1932 was chosen instead since it coincides with the beginning of a new period of static freight rates), this boundary has been located as shown by the new position on figure 6. Lincoln, Nebraska, will be the representative origin on this boundary.

TABLE 2

AGGREGATED RECEIPTS* OF BUTTER BY MARKET GROUPS, 1925-1950

\begin{tabular}{|c|c|c|c|c|c|c|}
\hline \multirow[b]{2}{*}{ Year } & \multicolumn{4}{|c|}{ Receipts at four markets $\dagger$} & \multicolumn{2}{|c|}{$\begin{array}{l}\text { Receipts at Los Angeles } \\
\text { and San Francisco }\end{array}$} \\
\hline & $\begin{array}{c}\text { Origins in } \\
\text { Mountain and } \\
\text { Pacific states }\end{array}$ & $\begin{array}{c}\text { Origins in } \\
\text { Mountain and } \\
\text { Pacific states } \\
\text { excluding } \\
\text { Colorado }\end{array}$ & $\begin{array}{l}\text { Origin } \\
\text { in } \\
\text { Colorado }\end{array}$ & $\begin{array}{l}\text { Total } \\
\text { receipts }\end{array}$ & $\begin{array}{c}\text { Origins other } \\
\text { than } \\
\text { Mountain and } \\
\text { Pacific states }\end{array}$ & $\begin{array}{l}\text { Total } \\
\text { receipts }\end{array}$ \\
\hline & \multicolumn{6}{|c|}{ thousand gross pounds } \\
\hline 1925. & 1,058 & 628 & 430 & 652,974 & 2,678 & 68,349 \\
\hline 1926. & 1,834 & 918 & 916 & 651,808 & 512 & 71,637 \\
\hline 1927. & 2,570 & 1,870 & 700 & 662,866 & 574 & 68,471 \\
\hline 1928 & 3,465 & 1,074 & 2,391 & 652,927 & 209 & 67,487 \\
\hline 1929. & 2,348 & 821 & 1,527 & 678,961 & 442 & 67,504 \\
\hline 1930. & 2,221 & 1,299 & 922 & 657,925 & 2,107 & 68,754 \\
\hline 1931 . & 846 & 405 & 442 & 685,698 & 122 & 73,605 \\
\hline 1932 . & 474 & 325 & 149 & 680,174 & 548 & 72,988 \\
\hline 1933. & 1,572 & 731 & 841 & 732,111 & 710 & 70,326 \\
\hline 1934 & 1,665 & 1,054 & 611 & 670,979 & 4,116 & 68,831 \\
\hline $1935 \ldots$ & 748 & 462 & 286 & 633,475 & 11,226 & 73,998 \\
\hline $1936 \ldots$ & 855 & 177 & 678 & 626,013 & 13,189 & 74,660 \\
\hline $1937 \ldots \ldots \ldots \ldots \ldots \ldots$ & 1,792 & 788 & 1,004 & 617,308 & 9,021 & 71,398 \\
\hline $1938 \ldots \ldots \ldots \ldots \ldots$ & 1,232 & 91 & 1,141 & 764,435 & 6,865 & 80,982 \\
\hline $1939 \ldots$ & 1,147 & 706 & 441 & 698,093 & 14,450 & 86,118 \\
\hline $1940 \ldots$ & 531 & 285 & 246 & 667,591 & 11,236 & 90,378 \\
\hline $1941 \ldots$ & 839 & 240 & 599 & 681,619 & 15,872 & 92,867 \\
\hline $1942 \ldots$ & 808 & 467 & 341 & 616,064 & 32,207 & 98,317 \\
\hline $1943 \ldots$ & 28 & 28 & 0 & 486,776 & 47,881 & 94,606 \\
\hline $1944 \ldots$ & 3,542 & 3,160 & 382 & 466,741 & 41,976 & 85,211 \\
\hline $1945 \ldots$ & 622 & 346 & 277 & 450,968 & 50,530 & 88,291 \\
\hline $1946 \ldots$ & 1,615 & 1,524 & 91 & 328,600 & 30,761 & 43,963 \\
\hline $1947 \ldots$ & 1,085 & 762 & 323 & 427,382 & 19,817 & 61,383 \\
\hline .......... & 228 & 201 & 27 & 396,792 & 24,833 & 59,353 \\
\hline $1949 \ldots$ & 158 & 150 & 8 & 418,330 & 22,932 & 61,217 \\
\hline $1950 \ldots \ldots \ldots \ldots \ldots \ldots$ & 48 & 24 & 24 & 403,548 & 20,397 & 61,047 \\
\hline
\end{tabular}

* Receipts include intermarket shipments.

+ Boston, New York, Philadelphia, and Chicago.

Source: U. S. Bur. Agr. Econ. Summary of Dairy and Poultry Statistics. Wash., D.C. (Annual.)

Expected and actual price differences. With the selected cities as reference points on the boundary locations, the left portion of table 3 has been computed to show the price differences that should have been expected between the various markets. The period 1921-1950 has been divided into intervals during which approximately static freight rates were in effect. This was necessary in order to eliminate the effect of rate changes on the expected price differences. To avoid making all the possible binary comparisons of prices, only the Chicago price was used as the base and all other prices related to it. The resulting differences permit all other possible comparisons to be made if so desired. Since no common boundary was postulated 


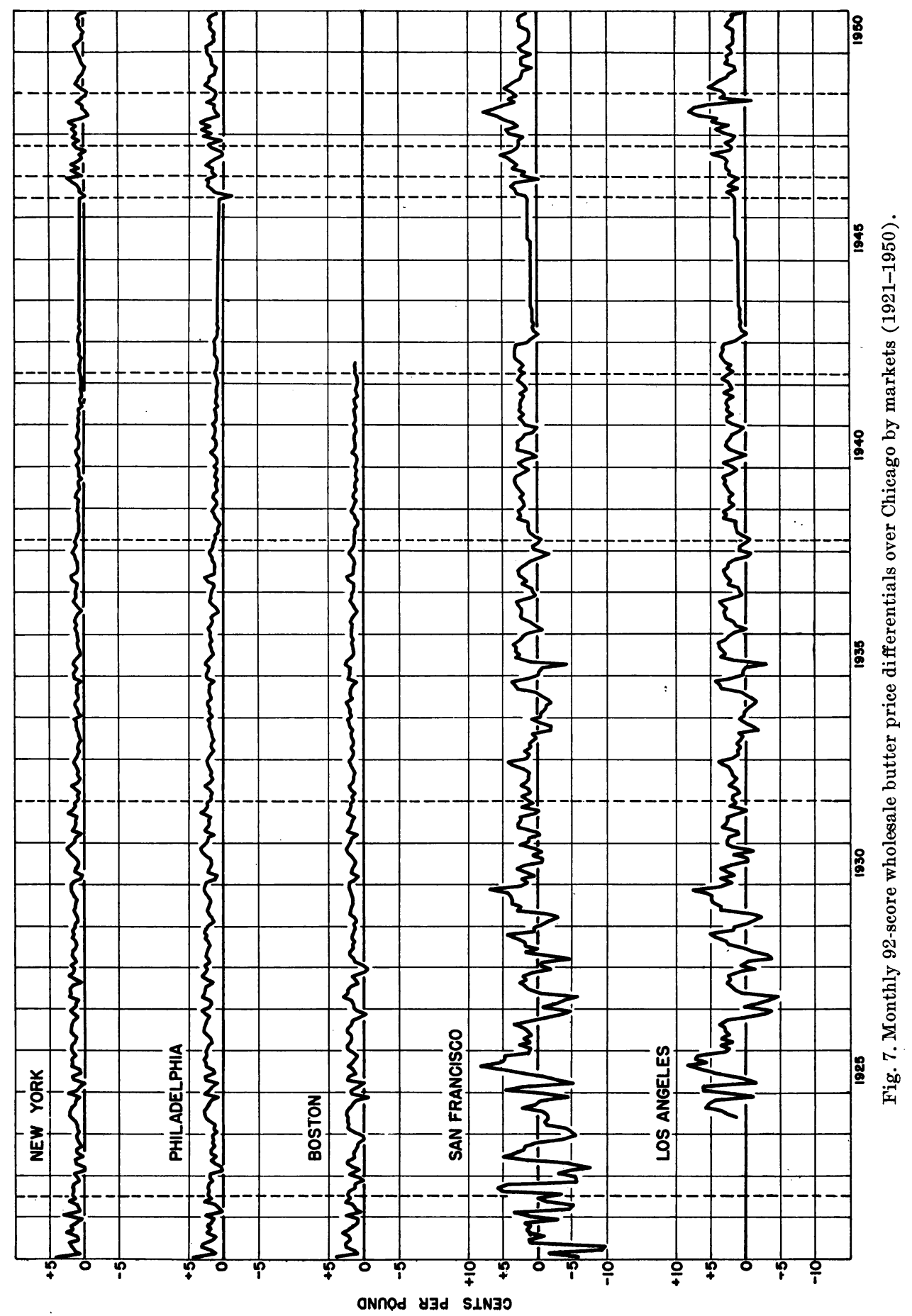


between the supply areas of the western markets and Chicago, the price difference at the former over the latter had to be determined by calculating the amount that the price at San Francisco (or Los Angeles) should exceed the price at New York and add to this the amount that the price at New York should exceed the price at Chicago.

TABLE 3

ESTIMATED* AND ACTUAL AVERAGE AMOUNTS BY WHICH BUTTER PRICES AT DESIGNATED MARKETS SHOULD HAVE EXCEEDED AND DID EXCEED THE PRICE AT CHICAGO

\begin{tabular}{|c|c|c|c|c|c|c|c|c|c|}
\hline \multirow[b]{2}{*}{$\begin{array}{l}\text { Intervals with nearly constant } \\
\text { freight rates }\end{array}$} & \multicolumn{4}{|c|}{ Estimated price differences } & \multicolumn{5}{|c|}{ Actual average price differences } \\
\hline & $\begin{array}{l}\text { New } \\
\text { York }\end{array}$ & $\begin{array}{l}\text { Phila- } \\
\text { delphia }\end{array}$ & Boston & $\begin{array}{c}\text { San } \\
\text { Francisco } \\
\text { and Los } \\
\text { Angeles }\end{array}$ & $\begin{array}{l}\text { New } \\
\text { York }\end{array}$ & $\begin{array}{l}\text { Phila- } \\
\text { delphia }\end{array}$ & Boston & $\begin{array}{c}\text { San } \\
\text { Fran- } \\
\text { cisco }\end{array}$ & $\begin{array}{c}\text { Los } \\
\text { Angeles }\end{array}$ \\
\hline & \multicolumn{9}{|c|}{ cents per pound } \\
\hline Jan. 1921-June 1922. & 1.08 & 1.06 & 1.14 & $\dagger$ & 1.60 & 2.04 & 1.88 & $\dagger$ & $\dagger$ \\
\hline July 1922-Dec. 1931 . . & .97 & .95 & 1.02 & $\dagger$ & 1.31 & 2.08 & 1.42 & $\dagger$ & $t$ \\
\hline Jan. 1932-Mar. 1938. . & .71 & .67 & .72 & 1.11 & .98 & 1.81 & 1.66 & .93 & 1.45 \\
\hline Apr. 1938-Mar. 1942. & .74 & .70 & .75 & 1.14 & .66 & 1.05 & 1.13 & 1.99 & 2.11 \\
\hline Apr. 1942-June 1946. & .79 & .74 & .78 & 1.19 & .74 & .83 & $\ddagger$ & 1.38 & 1.39 \\
\hline July 1946 -Dec. 1946 . . & .77 & .72 & .76 & 1.17 & .99 & 1.51 & $\ddagger$ & 2.48 & 1.73 \\
\hline Jan. 1947-Sept. 1947. . & .90 & .84 & .88 & 1.30 & .81 & 1.40 & $\ddagger$ & 3.37 & 2.70 \\
\hline Oct. 1947 -Dec. 1948. & 1.14 & 1.08 & 1.12 & 1.55 & .75 & 1.87 & $\ddagger$ & 4.11 & 3.73 \\
\hline Jan. 1949 -Dec. 1950. & 1.19 & 1.13 & 1.18 & 1.60 & .50 & 1.69 & $\ddagger$ & 2.31 & 2.27 \\
\hline
\end{tabular}

* The amounts shown include estimated differences in icing and switching costs as well as differences in the basic transportation rates from estimated boundaries of the actual supply areas.

$\dagger$ Not computed because of seasonal variation in supply area boundary.

$\ddagger$ Not available because of incomplete price series.

Source: For left portion: Basic freight rates secured from Dairy and Poultry Yearbook, Dairy Listing Service (Chicago, Ill.) and a Southern Pacific Railroad agency. Icing costs and switching charges for 1946-1950 approximated by the latter source. Prior years only estimated by the author.

For right portion: Compiled price series.

The actual price differences at the various markets over the price at Chicago are given on figure 7. Vertical broken lines on this figure indicate points when major rate changes occurred. In addition to figure 7 , the right portion of table 3 shows the simple average of the actual price differences during the same time intervals. Using table 3, one can compare expected and actual average price differences in order to judge the spatial efficiency of the butter market. This leads to an interpretation of the results thus far.

Before interpreting the geographic price structure of the butter market, a problem of causal implication must be discussed. In the simple case of considering the price differences over time at two markets, the problem is exposed. Let it be assumed that the actual price difference is too great compared with the expected amount based on transportation and handling costs. Without a knowledge of the f.o.b. plant prices at the actual common supply area, it cannot be ascertained whether a given market is or whether both are the cause of the discrepancy. One market price could be too high, the other could be too low, or both could be either too high or too low, but in differing amounts. A further possibility is that the actual common supply area is not located efficiently because of imperfect knowledge possessed by buyers and sellers, especially the latter. In the interpretation that follows, no assessing of 
responsibility for observed discrepancies will be made except in those cases where the evidence justifies doing so.

Interpretation of results. As shown in figure 7 and table 3, the New York and Chicago butter prices have generally moved relative to one another to maintain actual differences approximately compatible with what one would have expected. It is doubtful that better accord should be expected with potentially inaccurate basic data and possible failure of assumptions being true. Nevertheless, it seems that the New York price may have been slightly too high before 1947 and too low thereafter.

The case of Philadelphia is more obvious. The price of butter in that city has not only been too high relative to Chicago but also relative to New York and Boston. This conclusion is based only on the price analysis. Supplementary information given in the literature seems to negate this glaring difference. It appears that 92-score butter is graded more strictly in Philadelphia than in most other markets (Cassels, 1932). A letter, dated February 8, 1952, from H. A. Rust, Assistant Chief; Dairy and Poultry Market News Division, indicates that score qualities (color, odor, salt, et cetera) are somewhat unique in Philadelphia. Also, many small lot sales are included in the wholesale category. A grade differential of one cent is not unreasonable, on this basis, so the Philadelphia butter price may have been generally consistent with price movements in Chicago and the other eastern markets. The validity of this interpretation rests, of course, on the authenticity of the stricter grading argument.

The price series for butter at Boston, which was used, failed to cover the full period from 1921 through 1950 but ended in 1942. For the four time intervals available, the Boston price was probably slightly high relative to Chicago and to a greater extent than the New York price. As in the case of New York and Philadelphia (with the assumed grade difference), the deviations are not believed to be of sufficient magnitude to indicate serious inefficiency in the pricing mechanism.

A consideration of the prices at the two western markets leads to a different situation from that existing for the eastern markets. Prior to 1932, the Mountain and Pacific states were nearly self-sufficient in butter production. In fact, some of the more easterly states of this set were shipping to both eastern and western markets. The flush season of butter production in the Great Basin area and the coastal states comes a little earlier in the calendar year than in the Midwest. During that part of the year, the volume of production led to surpluses that tended to depress western prices rather greatly. The aggregated total of these excesses was probably quite large relative to the West Coast demand of that time but was a rather insignificant volume of short continuity when viewed by the eastern markets. This meant that the initiating of buyer-seller contacts for this surplus butter required a depressed western price. During the latter half of the year, a slight deficit condition arose in the western states, and a rather high price was required in order to induce inshipments. This led to a seasonally oscillating negative and positive difference in the price at the western markets over the price in Chicago with rather large amplitude. Although the actual data show this rather imperfectly in figure 7 , there is a tendency for the differences to be negative during the first half 
and positive during the last half of the years in the period 1921 through 1931. Later, the area became essentially a fully deficit region, and the price differences began to stabilize at a positive level.

Referring to table 3 again, the price in San Francisco seems to have moved rather efficiently during the first three effective time intervals. Comparisons for the last four time intervals (especially the first three of these) for the San Francisco market are believed to show a significantly too high butter price over Chicago. It is further believed that the eause of the discrepancy lies in the San Francisco price. Secondary information for San Francisco suggests two causes for the high price situation: (1) the transactions upon which the wholesale prices are based are probably heavily weighted by local brand sales reflecting a nonscore premium; and (2) the grading of butter is probably stricter in the western markets than at Chicago. However, there seems to be no logical reason why these two situations should be true recently and not earlier.

The Los Angeles butter price also appears to have been generally too high relative to the Chicago price. Prices during the third, fifth (price-controlled period), and sixth time intervals might be excluded from this judgment. Discrepancies during the last three intervals are not so great as for San Franciseo. The secondary information given in the discussion of the San Francisco price most likely applies also in the Los Angeles situation.

From the above considerations of the butter prices, market by market, certain minor imperfections were pointed out. Most of these imperfections were reduced in importance through supplementary information, but it is not denied that actual inefficiency in price formation in some cases may have existed. Since the supplementary information cannot easily be quantified, the amount of residual discrepancy cannot be determined. As a generalized conclusion, it is believed that the residual discrepancy is not large enough to be significant and that the geographic structure of butter prices has manifested reasonable efficiency for the pricing mechanism.

\section{B. Analysis of the Geographic Structure of American Cheese Prices}

Whole milk American cheese of the cheddar type has been in the past and is currently being produced in numerous states of the United States. However, production is far less widespread for cheese than for butter, with rather marked concentration in a few areas. Of the four or five leading states in cheese production, Wisconsin has been singularly dominant throughout the period of this study, 1921-1950. Due to this dominance and its central location in the United States, Wisconsin is the source of large quantities of cheese for all the leading consumption centers of the country. Data on origin of receipts indicate that this state can be used as a supply point for at least marginal volumes for all the markets (New York, Boston, Philadelphia, Chicago, San Francisco, and the Wisconsin Cheese Exchange at Plymouth, Wisconsin) considered in this analysis. This obviates the problem of locating intermarket boundaries - they are all assumed to focus on Wisconsin. Plymouth, Wisconsin, will be chosen as the origin point.

Assumptions. United States Department of Agriculture price quotations for cheese at the markets listed above are used in this analysis. These whole- 


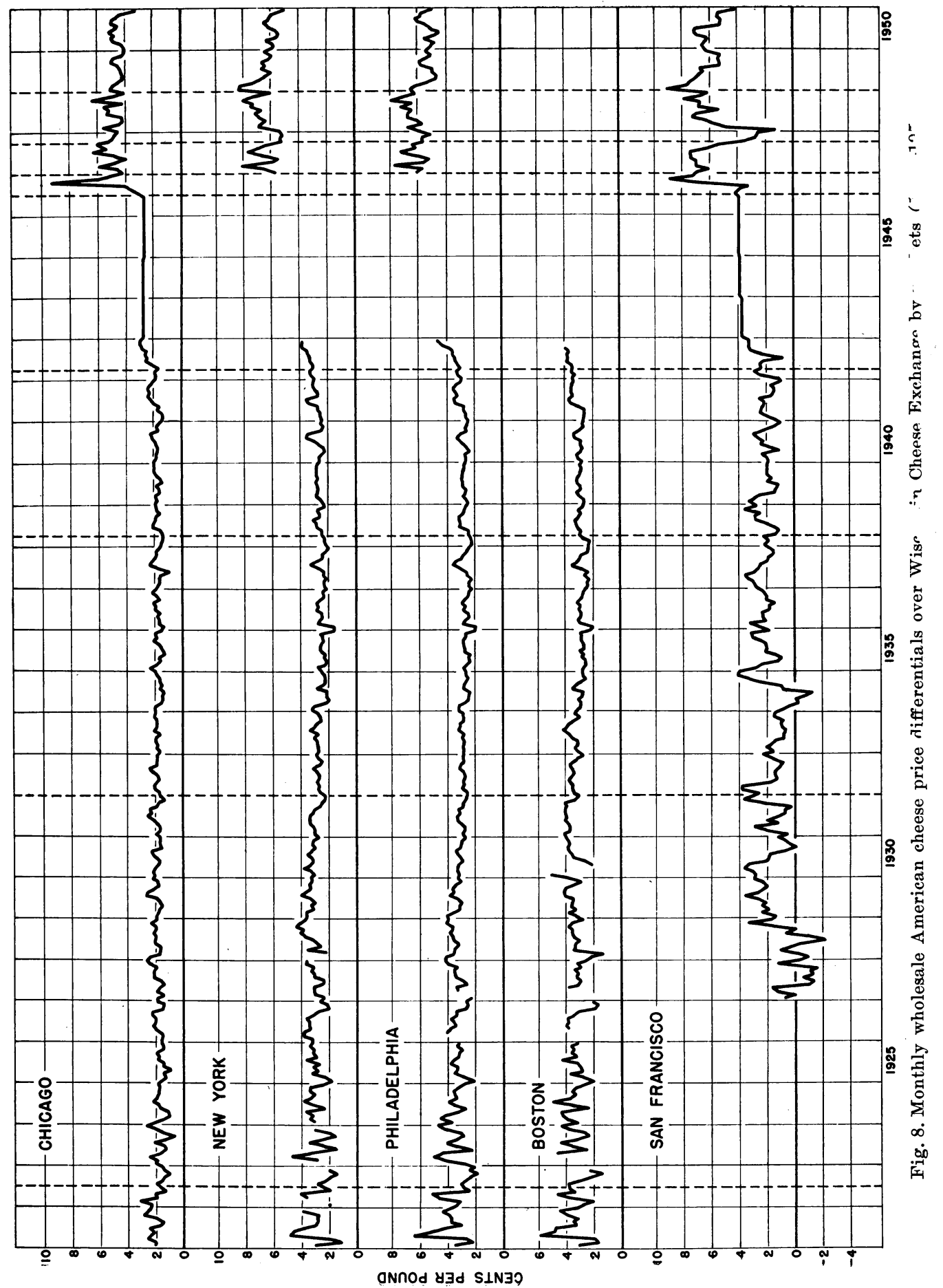


sale prices refer to the same level of the marketing channel as mentioned before for butter, and a similar assumption is implied for margins here. A further complication arises because of the numerous styles in which cheese is formed. Lack of availability forced a use of prices which are not universally for a single style, that is, prices in some markets are for Single Daisies, in others for Flats, Twins, or Triplets. It does not seem essential to define styles here but, to inform the reader, these names refer to individual shapes and/or types of package. The assumption that the influence of style on price is negligible, compared with other differences to be considered, will be made as an initial simplification. Although the prices used are supposedly for fresh cheese and will be taken as such, it is likely that sizable volumes of the aged product enter into the transactions upon which the price data are based-especially at all markets except the Wisconsin Cheese Exchange. The differing characteristics of summer and winter cheese (called grass and fodder cheese, respectively) should not enter as a factor causing heterogeneity since the price data are for shorter time intervals.

Just as the quality or grade of butter varies by a score rating, cheese of different lots and from different plants shows considerable variation. Since the average price quotations used do not specify the quality or grade of the cheese, it is difficult to account for this factor. On the basis of other writings (Miller, 1949), it will be assumed that the quality of cheese traded on the Exchange normally represents a lower grade product than on the other markets. An equivalent average grade will be assumed for all the other markets.

Expected and actual price differences. Using Plymouth, Wisconsin, as an origin point for supply, the left part of table 4 has been computed from freight rates and estimated service charges to show expected price differences at various markets over the price at the Exchange during constant rate time intervals. Since freight rates are for gross poundage, including package weight, and product prices are values for the net product weight, the freight rates were corrected to a net product weight basis. The correction factors were 1.03 for butter, $\mathbf{1 . 0 9}$ for cheese, and 1.21 for evaporated milk. These factors were taken from Vial (1950). Figure 8 shows the actual price differences; and the right part of table 4 , the average of these differences during the same intervals as mentioned above.

Interpretation of results. The marked lack of equivalence between the left and right portions of table 4 requires a partial interpretation before proceeding. A slight increase of the noticeably too low price at the Exchange can be made by including the assembly charges on carlot sales of $3 / 8$ cents per pound (1931-1938), 5/8 cent per pound (1938-1947), and $7 / 8$ cent per pound (19471951), which were permitted over the Exchange price as quoted. However, these amounts account for only a part of the large discrepancy. A few qualitative arguments will be presented now as partial explanation of the inconsistent level of the Exchange price relative to the other markets. It is not implied that they are sufficient for this purpose. First, the Exchange price is for Twins, which normally is lower by a fraction of a cent per pound than the Single Daisy price. Second, a possible inclusion of some aged cheese in the transactions at the other markets would raise their prices slightly over that of the Exchange. Third, the Exchange's transactions are probably quite 
heavily weighted with distress sales and an average lower quality type of cheese than traded at other markets. The combined effect of all the above factors might have been sufficient to explain the differences during the first five time intervals but are not believed sufficient for the remaining intervals.

The above limitations apply to the Plymouth quotations. Do any of the remaining markets exhibit prices that are inconsistent within the reduced group ? Still using Plymouth as the unique origin point of supply but Chicago

TABLE 4

\section{ESTIMATED AND ACTUAL AVERAGE AMOUNTS BY WHICH AMERICAN CHEESE PRICES AT DESIGNATED MARKETS SHOULD HAVE EXCEEDED AND DID EXCEED THE PRICE AT THE WISCONSIN CHEESE EXCHANGE}

\begin{tabular}{|c|c|c|c|c|c|c|c|c|c|c|}
\hline \multirow[b]{2}{*}{$\begin{array}{l}\text { Intervals with nearly } \\
\text { constant freight rates }\end{array}$} & \multicolumn{5}{|c|}{ Estimated price differences } & \multicolumn{5}{|c|}{ Actual average price differences } \\
\hline & Chicago & $\begin{array}{l}\text { New } \\
\text { York }\end{array}$ & $\begin{array}{l}\text { Phila- } \\
\text { delphia }\end{array}$ & Boston & $\begin{array}{c}\text { San } \\
\text { Fran- } \\
\text { cisco }\end{array}$ & Chicago & $\begin{array}{l}\text { New } \\
\text { York }\end{array}$ & $\begin{array}{l}\text { Phila- } \\
\text { delphia }\end{array}$ & Boston & $\begin{array}{c}\text { San } \\
\text { Fran- } \\
\text { cisco }\end{array}$ \\
\hline Jan. 1921-June 1922. & 0.44 & 1.13 & 1.11 & 1.19 & 2.62 & 2.15 & 3.06 & 3.81 & 3.58 & $*$ \\
\hline July 1922-Dec. 1931. & .40 & 1.04 & 1.01 & 1.09 & 2.39 & 1.82 & 3.10 & 3.30 & 3.40 & 1.22 \\
\hline Jan. 1932-Mar. 1938 & .32 & 0.81 & 0.79 & 0.87 & 1.91 & 1.78 & 2.56 & 2.71 & 2.99 & 1.85 \\
\hline Apr. 1938-Mar. 1942. & .35 & .88 & .86 & .90 & 2.07 & 1.88 & 2.79 & 2.84 & 3.05 & 2.02 \\
\hline Apr. 1942-June 1946 & .38 & .92 & .89 & .94 & 2.15 & 2.70 & $*$ & $*$ & $*$ & 3.65 \\
\hline Jan. 1949-Dec. 1950. & 0.63 & 1.54 & 1.49 & 1.58 & 3.29 & 4.53 & 6.30 & 5.31 & $*$ & 6.23 \\
\hline
\end{tabular}

* Not available because of incomplete price series.
Source:
For left portion: Basic freight rates from Cassels, J. M. Study of Fluid Milk Prices (Cambridge, Mass., 1939,
p. 261), and a Southern Pacific Railroad agency.
For right portion: Compiled price series. For right portion: Compiled price series.

as the base market, table 5 was computed to answer this question. The latter table was secured directly from the values in table 4.

Considering New York, Philadelphia, and Boston together, rather good accord is found between the expected and actual average price differences over Chicago. There is a tendency for these three markets consistently to have a too high price relative to Chicago, but the amount is generally not very large. It is possible that the polyglot population of the eastern cities demands a higher grade of natural cheese than the midwestern people and that a grade differential is responsible for the observed discrepancy.

In contrast to the case of the eastern markets, a somewhat stronger and opposite tendency for divergence of the San Francisco price from what should have been expected over the Chicago price is observed from the two relevant columns of table 5. Part of the relatively low condition of the San Francisco price can be attributed to a style difference for the price quotations. This market quoted prices on Flats until 1943 and on Triplets thereafter. Usually these styles are sold at less than one cent per pound under the Single Daisy price which is the style for Chicago prices. No further arguments are known to explain the residual amount of the low difference. 
Excepting the Wisconsin Cheese Exchange prices, the geographic structure of cheese prices at the other markets considered in this analysis seems to indicate rather efficient results for the pricing mechanism. Excluding postWorld War II operations, the actual volumes traded on the Exchange were very small. The Exchange prices did move consistently with the prices in the other major markets (or vice versa) but probably at a consistently too low level unless the previous qualifying arguments negate this judgment.

TABLE 5

ESTIMATED AND ACTUAL AVERAGE AMOUNTS BY WHICH AMERICAN CHEESE PRICES AT DESIGNATED MARKETS SHOULD HAVE EXCEEDED AND DID EXCEED THE PRICE AT CHICAGO

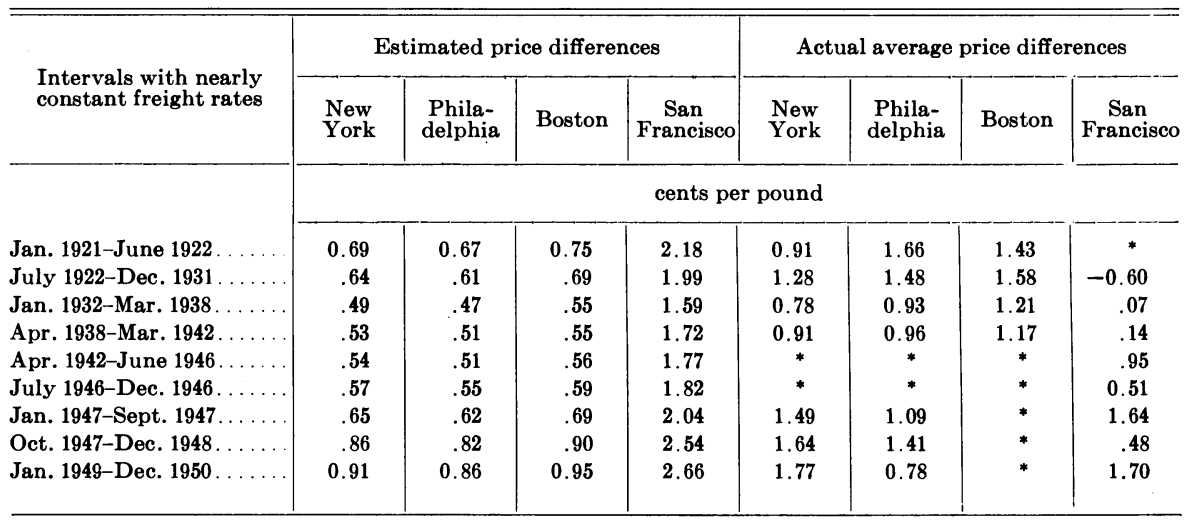

* Not available because of incomplete price series.

Source:

For left portion: Basic freight rates from Cassels, J. M., Study of Fluid Milk Prices (Cambridge, Mass., 1939, p. 261), and a Southern Pacific Railroad agency.

For right portion: Compiled price series.

\section{Analysis of the Geographic Structure of Evaporated Milk Prices}

Evaporated milk, because of its high weight per unit of milk equivalent, has a rather extensive geographic distribution of production. This distribution is not so continuous as in the case of butter production but is highly concentrated in spots. The large volumes of milk necessary for plant operations prevent the economical production of evaporated milk in areas of low density for the farm supply of milk. Table 6 has been computed for the years 1930 through 1949 to show in a general way the location of surplus and deficit production areas. Approximately the same situation existed for the preceding decade, except that the North Atlantic area was of greater relative importance in production.

The North Atlantic states (New England and Middle Atlantic), South Atlantic states, and the South Central states have been deficient in local production although production has been increasing significantly in the last two of these areas. Per capita consumption has been higher in the South Central region than the average for the United States, so that area has been included in the deficit group. The West North Central region was probably deficit prior to 1940 and a surplus area thereafter. The Western states and the East North Central group have been consistently surplus areas of pro- 
duction. It should be stressed that these are only broad conclusions and that variation in surplus and deficit conditions within these areas have been and are presently as large and significant as between the areas.

Product differentiation by brands. Before analyzing the geographic price structure for evaporated milk, it is necessary to consider some background information. Evaporated milk is a highly standardized dairy product, and all plants adhere very closely to established requirements. Primarily because

TABLE 6

PER CAPITA REgIONAL PRODUCTION AND UNITED STATES CONSUMPTION OF EVAPORATED MILK

(Case Goods)

\begin{tabular}{|c|c|c|c|c|c|c|c|}
\hline Year & $\begin{array}{c}\text { North } \\
\text { Atlantic }\end{array}$ & $\begin{array}{c}\text { East } \\
\text { North } \\
\text { Central }\end{array}$ & $\begin{array}{c}\text { West } \\
\text { North } \\
\text { Central }\end{array}$ & $\begin{array}{c}\text { South } \\
\text { Atlantic }\end{array}$ & $\begin{array}{c}\text { South } \\
\text { Central }\end{array}$ & Western & $\begin{array}{c}\text { United } \\
\text { States } \\
\text { consump- } \\
\text { tion }\end{array}$ \\
\hline & \multicolumn{7}{|c|}{ pounds per capita } \\
\hline 1930. & 3.11 & 32.12 & 6.44 & 0.83 & 4.09 & 28.34 & 11.2 \\
\hline $1931 \ldots$ & 3.15 & 31.39 & 5.62 & 0.87 & 4.27 & 27.78 & 11.4 \\
\hline 1932. & 3.69 & 34.79 & 5.98 & 0.91 & 4.93 & 28.35 & 12.3 \\
\hline 1933. & 3.31 & 39.22 & 6.37 & 1.00 & 4.91 & 30.91 & 12.3 \\
\hline $1934 \ldots \ldots \ldots$ & 2.99 & 40.23 & 6.11 & 1.12 & 5.02 & 28.53 & 13.4 \\
\hline $1935 \ldots \ldots \ldots$ & 4.10 & 42.47 & 6.49 & 1.55 & 5.51 & 28.05 & 14.6 \\
\hline $1936 \ldots$ & 5.68 & 46.43 & 7.62 & 1.43 & 5.99 & 28.53 & 14.0 \\
\hline 1937. & 4.29 & 42.04 & 8.83 & 1.51 & 7.00 & 26.00 & 14.9 \\
\hline 1938. & 4.47 & 45.29 & 10.33 & 2.39 & 8.32 & 28.13 & 15.5 \\
\hline 1939. & 4.75 & 45.97 & 10.55 & 2.48 & 7.80 & 30.18 & 16.2 \\
\hline 1940 & 5.46 & 51.62 & 13.55 & 2.94 & 7.69 & 33.77 & 17.4 \\
\hline $1941 \ldots$ & 7.03 & 69.43 & 18.64 & 4.53 & 10.40 & 37.94 & 16.7 \\
\hline 1942. & 7.17 & 75.38 & 21.01 & 5.60 & 11.49 & 38.16 & 16.3 \\
\hline 1943 & 4.66 & 67.40 & 20.48 & 4.52 & 9.84 & 31.91 & 17.1 \\
\hline $1944 \ldots$ & 4.00 & 72.55 & 25.55 & 6.10 & 12.99 & 37.97 & 13.7 \\
\hline $1945 \ldots$ & 4.49 & 79.61 & 28.96 & 7.62 & 16.06 & 37.57 & 16.2 \\
\hline 1946. & 1.77 & 58.13 & 24.06 & 6.22 & 14.34 & 30.92 & 17.0 \\
\hline $1947 \ldots$ & 2.16 & 57.94 & 25.76 & 7.51 & 16.01 & 29.95 & 18.1 \\
\hline $1948 \ldots$ & 3.21 & 57.58 & 26.09 & 8.19 & 16.99 & 32.00 & 18.3 \\
\hline $1949 \ldots$ & 2.82 & 41.42 & 20.89 & 7.64 & 17.49 & 26.38 & 17.7 \\
\hline
\end{tabular}

Sources: Per capita production was computed from production data issued by the Bureau of Agricultural Economics and population data issued by the Bureau of the Census. Per capita consumption figures were taken from U. S. Bur. Agr. Econ., Changes in the Dairy Industry, United States, 1920-1950, Wash., D.C., July, 1950. (Table 74, p. 136.)

of a lack of knowledge on the part of consumers about the product and of advertising directed toward this lack of understanding, evaporated milk is an artificially differentiated product. (It is probably true that, in the years before and shortly after World War I, the product of firms with national distribution was more dependable and consistent in quality than the output of firms with only small-scale, local distribution. However, with the spread of technical ability, it is doubtful that significant differences in the physical product have existed since 1930. Prior to this, the production of local brands was probably quite small, excluding that which was contractually produced for a national brand firm.) The differentiation is manifested in the brand names of the producing firms. Pricewise, this differentiation separates the product into two distinct groups, namely, national brands and local brands, 
with the former selling at a varying premium rate over the latter. The term national brands refers to the output of firms with distribution of national scope, such as Carnation, Bordens, Pet, and Nestles. Local brands refers to the output of firms that must sell at a price below that of the national brands in order to secure a reasonable degree of acceptance by consumers. Some of the national brand companies also produce a secondary, local brand. Included in the category of local brands are the brands distributed through national chain stores at lower than national brand prices. Although this dichotomous grouping is not clearly observed under all marketing conditions, it is generally so.

Marketing agreements and zonal pricing. Before September, 1933, and after June, 1947, manufacturers' selling prices were essentially free market prices, except for any intentional or unintentional collusion which may have existed. The latter condition most likely prevailed for the national brand segment in which the share of total sales accruing to an individual firm was quite large. From September, 1933, through May, 1935, the industry operated under Marketing Agreement 7 of the AAA which was designed to establish minimum and maximum prices for selling evaporated milk, as well as to set minimum paying prices for milk used. Six geographic sections were established for the country with formulas set for paying for the milk by utilization. Prices actually paid were always above these minimum prices so the latter were never effective. Reference to these price formulas will be made in a later section. The selling prices were set f.o.b. the manufacturers' distribution points and were uniform within each of three zones established for the entire country. Both minimum and maximum prices were established for each zone, with certain designated points at which overages (markups) were allowed. Quoted prices were on carlot sales, and a five cents per case increase was permitted for l.c.l. sales. Other trade practice provisions were also specified.

From May, 1935, through June, 1947, the industry operated under Marketing Agreement 60 and License 100. In the area of selling prices, an open listing provision was made. Manufacturers were still bound to zonal pricing f.o.b. distribution points but were free to make changes by submitting a price list that was eventually distributed to all other suppliers. During the war years, O.P.A. ceiling prices were the effective controls. Although a thorough study of the economic implications of the provisions embodied in the control agreements would be very interesting, it cannot be achieved in this paper (Baker and Froker, 1945). A few conclusions will be presented but no intensive analysis will be made.

Table 7 designates the three selling price zones in effect from September, 1933, through June, 1947, and the fixed prices for the period of operation under Agreement 7. Zone I was approximately equal to the North Central, South Central, and South Atlantic areas combined; Zone II was essentially the New England and Middle Atlantic areas; while Zone III was nearly equivalent to the Mountain and Pacific areas.

A few words are necessary on the nature of the available prices for evaporated milk. Average wholesale selling prices at manufacturers' distribution points for carlot sales have been reported by Geographic Divisions. Such 
averages include both national and local brand prices and probably represent a weighted average price during the years when the industry operated under Marketing Agreements 7 and 60. By comparing reported prices with those in table 7, it can be observed that the average prices for areas in a given zone are not equal to each other. The differences are presumably due to differences in relative volumes of local and national brand sales, at least from September, 1933, to June, 1935. There is some evidence that the average during other periods is merely the midpoint of the reported price range or a simple average

TABLE 7

MAXIMUM AND MINIMUM CARLOT SELLING PRICES FOR EVAPORATED

MILK F.O.B. DISTRIBUTION POINTS BY ZONES* UNDER MARKETING AGREEMENT 7

\begin{tabular}{|c|c|c|c|c|}
\hline \multirow{2}{*}{ Zone } & \multicolumn{2}{|c|}{$\begin{array}{l}\text { September } 9,1933 \text {, to } \\
\text { January } 10,1935\end{array}$} & \multicolumn{2}{|c|}{$\begin{array}{c}\text { January } 10,1935 \text {, to } \\
\text { June } 1,1935\end{array}$} \\
\hline & Maximum & Minimum & Maximum & Minimum \\
\hline & \multicolumn{4}{|c|}{ dollars per case $\dagger$} \\
\hline I. $\ldots \ldots \ldots \ldots \ldots \ldots$ & 2.60 & 2.45 & 2.80 & 2.45 \\
\hline II $\ldots \ldots \ldots \ldots \ldots \ldots \ldots \ldots \ldots \ldots \ldots \ldots$ & 2.70 & 2.55 & 2.90 & 2.55 \\
\hline 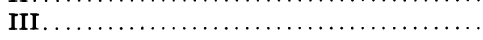 & 2.60 & 2.50 & 2.80 & 2.50 \\
\hline
\end{tabular}

* Zone I included all states not in Zones II and III. Overages of 10 cents per case were permitted for certain isolated points in Wyoming and New Mexico.

Zone II included Maine, New Hampshire, Vermont, Massachusetts, Connecticut, Rhode Island, New York, New Jersey, Delaware, Maryland, and approximately the eastern two thirds of Pennsylvania.

Zone III included Washington, Oregon, California, Idaho, Nevada, Utah, Arizona, and Montana. Overages of 10 cents per case were permitted for certain isolated points and all of Montana.

$\dagger 14 \frac{1}{3}$-ounce cans, 48 per case.
Source: U. S. Dept. of Agr., Agricultural Adjustment Administration, Marketing Agreement for Evaporated Milk, Wash., D.C., 1933; and Amendments.

of reported prices irrespective of volume. Actual pricing for each firm was constant for large zones (including surplus and deficit areas) so it will not require any analysis to show inefficiency in such procedures when compared with a competitive norm, since such pricing is inconsistent with the existence of transportation charges. A crude analysis can be made on a zonal basis, but it will be partially inconclusive because of a lack of knowledge pertaining to the composition of volumes upon which the regional prices were based. Nevertheless, one can take the East North Central area of Zone I as a surplus area, the New England and Middle Atlantic areas of Zone II as deficit areas, and the South Western Area of Zone III as a surplus area and make general price comparisons.

Using the East North Central price as a base, the relevant sections of figure 9 show the actual price differences for the areas designated above. Table 8 gives comparisons of expected and actual average price differences. In general, the information supplies evidence that the differences between prices in different zones were of the right sign but usually of the wrong magnitude.

Insofar as practically no shipment data for origin and destination of evaporated milk are available, little of a specific nature can be said about the observed price structure. However, a few reasonable arguments can be suggested from a general knowledge of surplus and deficit areas. Such arguments 
are presented only as possible descriptive explanations of past price results for the period during which the industry operated under marketing agreements.

The observed evaporated milk price structure. During the period 19331947, individual companies were required to have singular prices for all sales within each zone. Zone I, as previously designated, will be used in this argument. It will be noted that the East and West North Central, South Central, and nearly all of the South Atlantic areas are found in this zone. The question

TABLE 8

ESTIMATED AND ACTUAL AVERAGE AMOUNTS BY WHICH EVAPORATED MILK PRICES FOR DESIGNATED GEOGRAPHIC DIVISIONS SHOULD HAVE EXCEEDED AND DID EXCEED THE PRICE FOR THE EAST NORTH CENTRAL REGION

\begin{tabular}{|c|c|c|c|c|c|c|}
\hline \multirow{2}{*}{$\begin{array}{l}\text { Intervals with nearly constant } \\
\text { freight rates }\end{array}$} & \multicolumn{3}{|c|}{ Estimated price differenceз } & \multicolumn{3}{|c|}{ Actual average price differences } \\
\hline & N.E. & M.A. & S.W. & N.E. & M.A. & S.W. \\
\hline & \multicolumn{6}{|c|}{ cents per case* } \\
\hline Jan. 1921-June 1922. & 18 & 15 & 16 & 19 & 15 & 24 \\
\hline July 1922-Dec. 1931. & 15 & 13 & 14 & 15 & 11 & 11 \\
\hline Jan. 1932-Mar. 1938. & 13 & 11 & 12 & 8 & 5 & 8 \\
\hline Apr. 1938-Mar. 1942. & 14 & 12 & 13 & 10 & 6 & 5 \\
\hline Apr. 1942-June 1946 & 15 & 13 & 14 & 10 & 5 & 11 \\
\hline July 1946-Dec. $1946 \ldots$ & 16 & 14 & 15 & 10 & 5 & 5 \\
\hline Jan. 1947-Sept. 1947. . & 19 & 16 & 17 & 11 & 7 & 5 \\
\hline Oct. 1947-Dec. 1948. . & 23 & 19 & 22 & 13 & 9 & 14 \\
\hline Jan. 1949-Dec. $1950 \ldots$ & 26 & 21 & 23 & 9 & 8 & 18 \\
\hline
\end{tabular}

\footnotetext{
* 141/2-ounce cans, 48 per case.
}

Source:

For left portion: Basic freight rates from Cassels, J. M. Study of Fluid Milk Prices (Cambridge, Mass., 1939. p. 261), and a Southern Pacific Railroad agency.

For right portion: Compiled price series.

to be answered first is why the monthly average prices for each of these areas were not equal. Remembering that a dual pricing situation exists for evaporated milk, the higher average price in some areas of Zone I as compared with the East North Central region must have been due to a higher proportion of national brand sales in the former than in the latter. It appears that the southern areas were subject to this condition, while the West North Central region was not. The argument rests on the assumptions that the price of all national brands was uniform for the category and that a differentially lower price existed for the local brands. Both of these assumptions are apparently true.

The second argument is closely associated with the first. It involves a few implications of zonal pricing for the evaporated milk industry. If the product were not differentiated and the price f.o.b. plant were such as to be in competitive equilibrium with other co-located uses, an evaporated milk company would find it uneconomical as a continuous policy to establish distribution points in far distant deficit areas. The transportation costs could not be covered on movements from a plant in a surplus area to a distribution point 


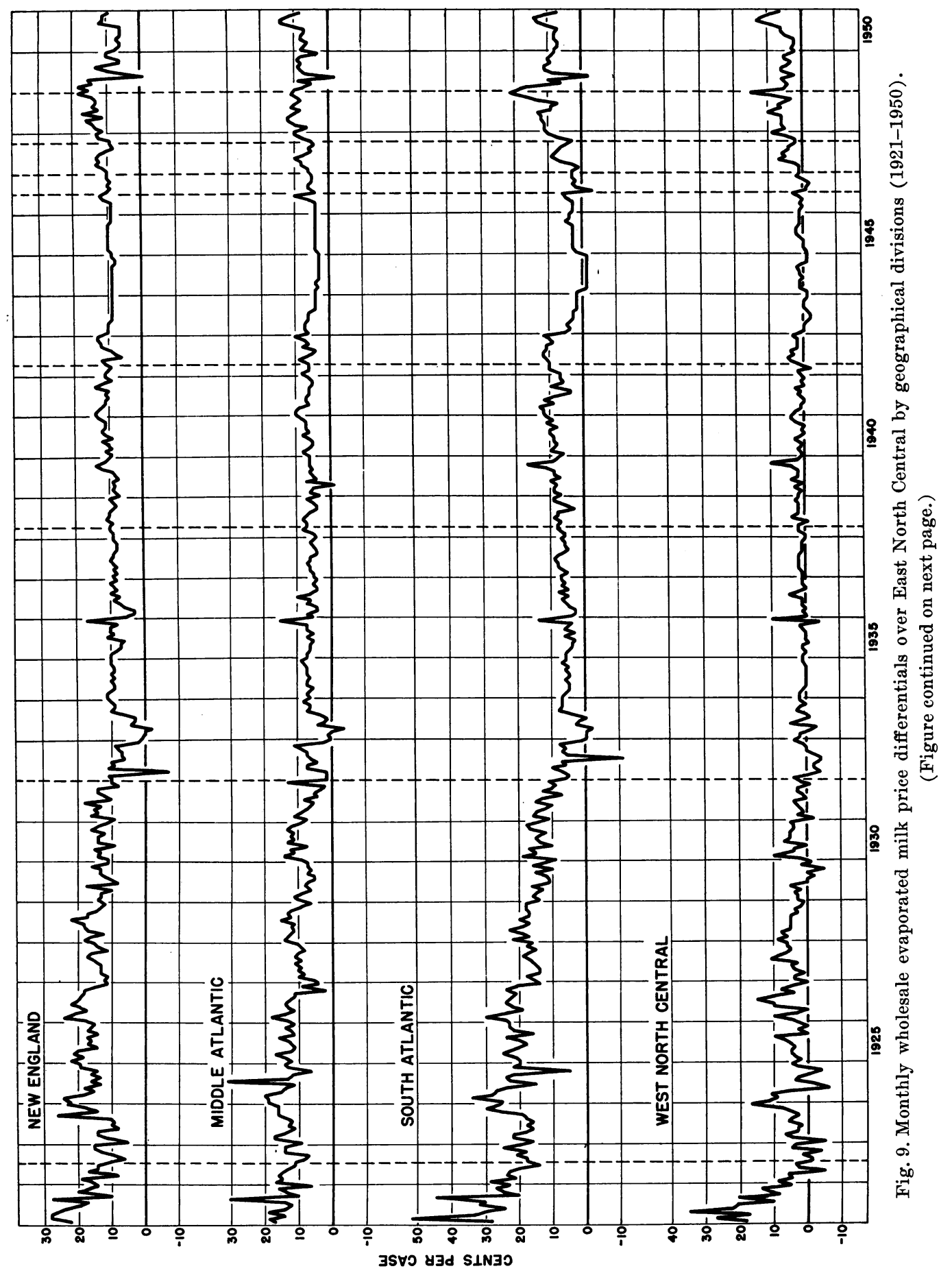




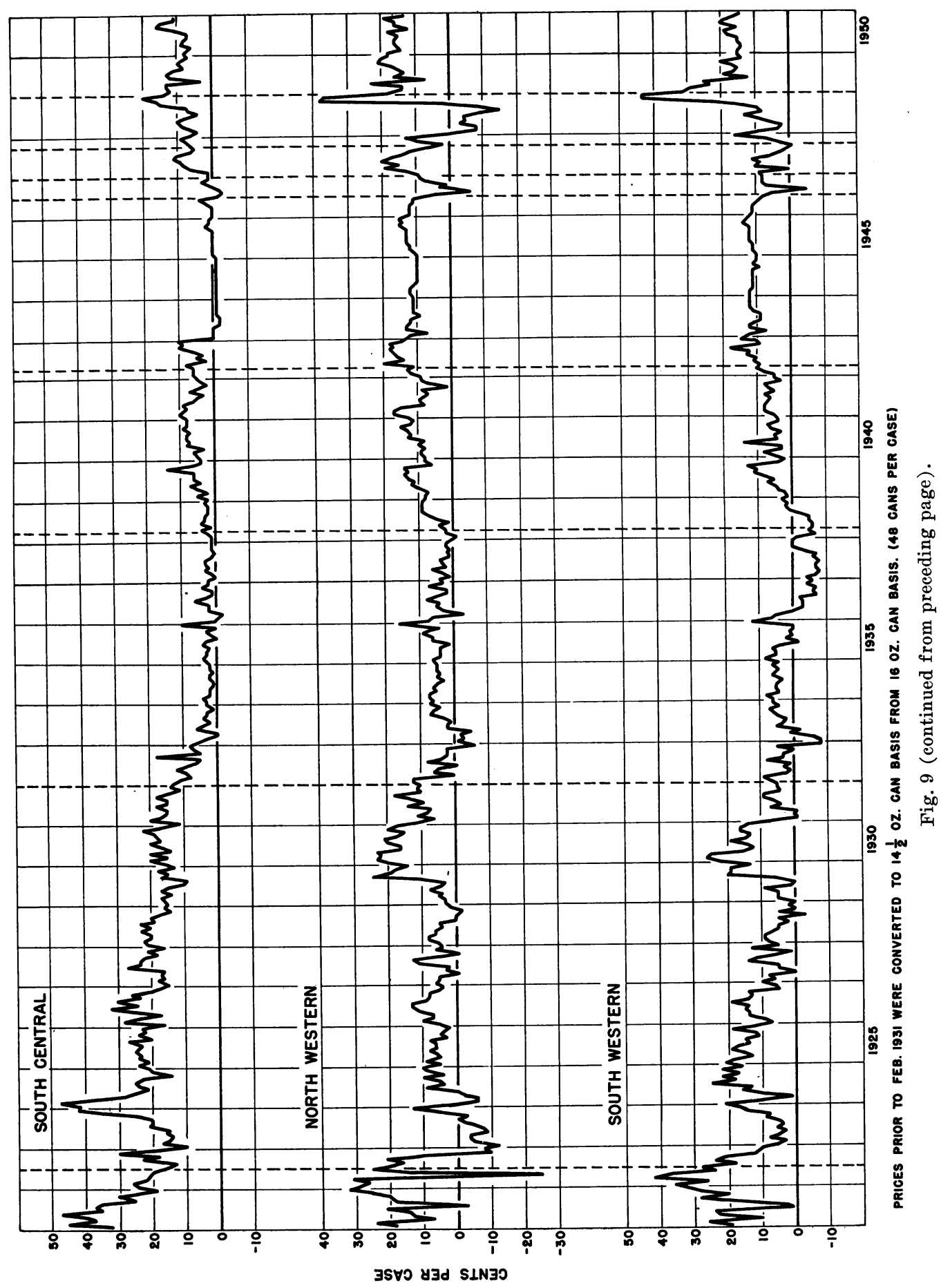


in a deficit area within the same zone under constant zonal pricing. Normally, one would expect that the distribution points would be the plant locations, and buyers would pay the shipping costs. Only through general collusive action by which the flat zonal price could be raised above the competitive level of the surplus area could far distant distribution points be maintained through time. This would involve a form of discriminatory pricing whereby the transportation costs on volumes sent to the distribution points in deficit areas would be covered (with or without excesses) by the slightly higher than competitive price on volumes sold in the surplus area.

Although this form of price discrimination would not be so effective with respect to profits as that which would result in equating the marginal returns in the two areas, it could result in excess aggregate profits if entry into production of evaporated milk was effectively barred and a workable agreement was reached on sharing the total sales return. With entry permitted, the long-run excess profits could decline to zero. The flat zonal price would be higher than the competitive level in the surplus area (and below in the deficit area) and would be an incentive for firms to break away from the sharing agreement based on allocating the transportation costs on the volumes shipped to the deficit area.

Now, this line of reasoning must be expanded to a setting that probably was (and still is) comparable with conditions in the surplus areas. In making the discussion more realistic, one must admit the very real existence of product differentiation, with only a few very large firms making up the national brand category. Assume that tacit collusion to avoid price competition held for the major firms and that local brand firms were competitive in their buying of raw milk and selling of their product. Further, major firms also produced some local brands, giving them partial means of controlling the volume of their major brand output so as not to break the latter's position in the market through excessive supply. This secondary output was either subcontracted or a regular line. It is also likely that, under conditions of rapidly expanding demand relative to national brand output capacity, the major firms let contracts to the local brand firms in order to increase their individual outputs by brand.

What might one expect major firms (in a surplus area of a given zone) to do in the realm of price policy within such an environment? The following sequence of propositions appears plausible: First, the competitive price for evaporated milk would be estimated from the opportunity value for milk in other uses. Second, the uniform national brand price for the zone would be set at a slightly higher price in response to the differential preferences exhibited by consumers. This difference must be less than the transportation cost of movements from surplus to deficit areas, where local production of evaporated milk does not occur, so as to insure the exclusion of local brand shipments from the surplus areas into the latter areas. It must be high enough to cover the transportation costs on those volumes shipped to distribution points in deficit areas-coverage being secured from surplus area sales. Third, it must be assumed that the long-run vision of a major firm would be in the direction of expanding its selling area even if this involved a short-run reduction in its profit rate. With this assumption and in the 
belief that the other major firms would not accept a given firm's singular expansion of this type, all the major brands would tend to foster establishment of distribution points in deficit areas. Fourth, and last, the major firms would attempt to establish plants in the deficit areas (subject to dairy production possibilities) in order to reduce the volumes that must be shipped from the surplus area.

Subsequent analysis of relative prices for the manufactured dairy products indicates that national brand prices for evaporated milk have been consistently higher than competitive levels. Local brand prices were more nearly competitive with butter and cheese. Also, firm expansions into deficit areas for production have been made almost entirely by national brand companies. All these facts support the line of reasoning given in the last paragraph.

\section{Use of Changes in Monthly Volume of Receipts at Various Markets to Test Efficiency of Reaction of Suppliers to Short-period Geographic Price Differences}

Previously it was pointed out, somewhat indirectly, that a large-scale shift occurred in the butter supply area for the western markets some time after the early 1930's. Upon subsequent analysis of price differences, it was generally conceded that such a shift was compatible with a rather sluggishly efficient reaction of suppliers to a geographic price differential. This conclusion was reached on the basis of average price differences for quite lengthy time intervals. Now it is desired to check whether the supplier reaction is efficiently responsive in short-period situations. For numerous reasons, the approach to this problem is necessarily weak. These reasons will be given later. The short-period analysis will apply only to butter and cheese and for one selected time interval.

The period 1932-1937 was one of rather stable, albeit low, product prices. It is believed that such a situation should lead to quite rapid reaction by suppliers to minor biased changes in price difference between markets, if such biases persisted over time. From figure 7 it can be observed that the New York, Philadelphia, and Boston butter prices moved with almost identical price changes since their differences over Chicago have very similar patterns. This was also true for cheese but to a lesser extent.

Method of analysis. Data are available on monthly receipts of butter and cheese at these four markets. Since these monthly volumes vary seasonally, it was difficult to use the absolute values to show supplier responses to price differences. The left portions of tables 9 and 10 have been computed to judge whether the total receipts at the four markets had a seasonal pattern equivalent to the United States production pattern. If this were true, then it could be assumed that the suppliers for these four markets remained stable (or that simultaneous movements in and out of this category balanced) during each season. Of course, this holds only if the seasonal patterns of the four market supply areas were the same as the national pattern. Before giving an interpretation of the results, the outlining of the procedure will be finished.

The basic idea involved a comparison of changes in the precentage of 
aggregated receipts at New York, Philadelphia, and Boston out of the total at the four markets, with changes in the price differences of these markets over Chicago. No lagged comparisons were made and, for simplicity only, the New York price difference was employed as an indicator. Also, the absolute amount of the change was not employed-instead, a plus sign was used to indicate an increase and a negative sign, a decrease in the New York price difference. These plus and minus signs are found under the relevant percentage figures of tables 9 and 10 .

\section{TABLE 9}

SHORT-PERIOD SUPPLIER RESPONSE TO CHANGES IN GEOGRAPHIC PRICE DIFFERENCES FOR BUTTER

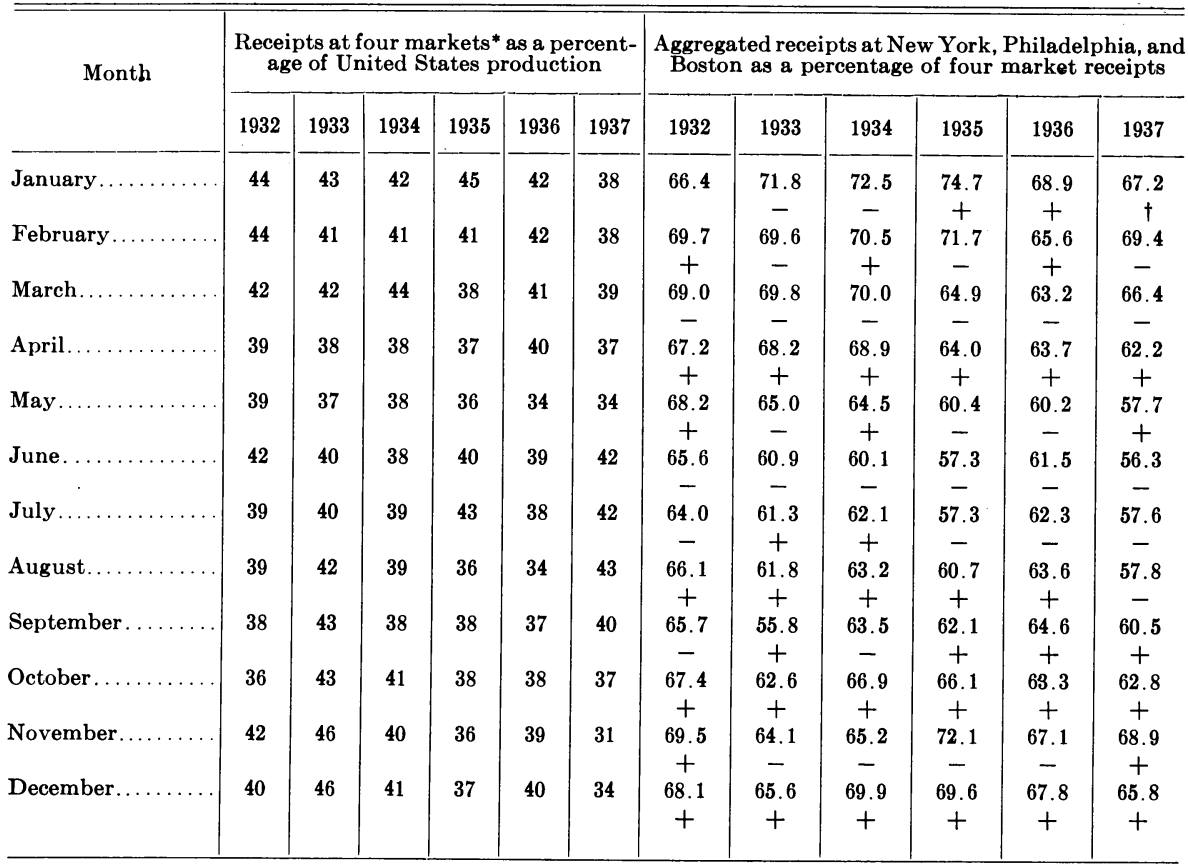

* New York, Philadelphia, Boston, and Chicago.

$\dagger$ No change. The plus (minus) sign indicates that the New York price difference over Chicago increased (decreased) over the preceding month's.

Source: U. S. Bur. Agr. Econ., Summary of Dairy and Poultry Statistics, Wash., D.C. (Annual issues.)

From the left portions of these tables, one can conclude that butter receipts at the four markets had a seasonal pattern approximately equivalent to the production pattern but that cheese receipts had less variation than production. Consequently, the stability of the four market supply area was greater for butter than cheese. The discrepancy for cheese may be due only to the manner by which cheese moves from plant to city market. Normally, a great percentage of the output is assembled by warehouse operators in the producing area prior to distribution to urban markets. With storage operations being carried on by the assemblers, the flow to markets is quite stable and 
exhibits little seasonal variation-being closely connected with the consumption rate.

Inconclusive results. The right portions of tables 9 and 10 were computed to indicate the consistency of supplier response to changes in the price differences of eastern markets over Chicago. The results show 42 consistent reactions for butter and 39 for cheese out of 71 months. Although this indicates a slight positive response by suppliers, it is far from conclusive.

TABLE 10

SHORT PERIOD SUPPLIER RESPONSE TO CHANGES IN GEOGRAPHIC PRICE DIFFERENCES FOR CHEESE

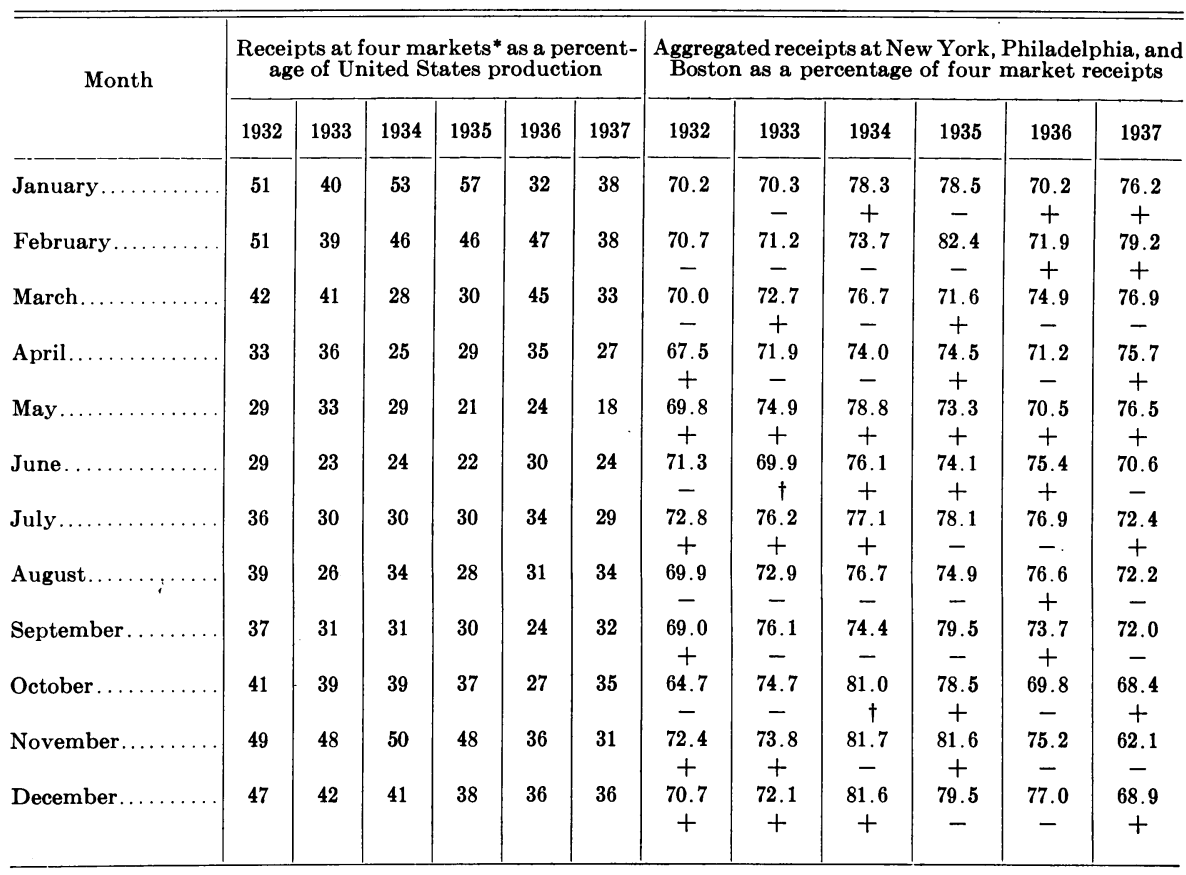

* New York, Philadelphia, Boston, and Chicago.

† No change. The plus (minus) sign indicates that the New York price difference over Chicago increased (decreased) over the preceding month's.

Source: U. S. Bur. Agr. Econ., Summary of Dairy and Poultry Statistics, Wash., D.C. (Annual issues.)

Reasons why this analysis may only be suggestive are given now. First of all, the results may be perfectly valid. At numerous points, it has been suggested that seller response to small erratic movements in price differences might not occur since costs are involved in making and breaking buyer-seller contacts. It is quite possible that the biased changes in the price difference from 1932-1937 were too small and did not continue long enough to elicit any noticeable response.

However, from the logical point of view, the analysis is weak. It is based only on the supply response and fails to account for the fact that the basic prices are a result of the interaction of the supply flow and the demand intensities. Conceivably, a situation like the following would be camouflaged 


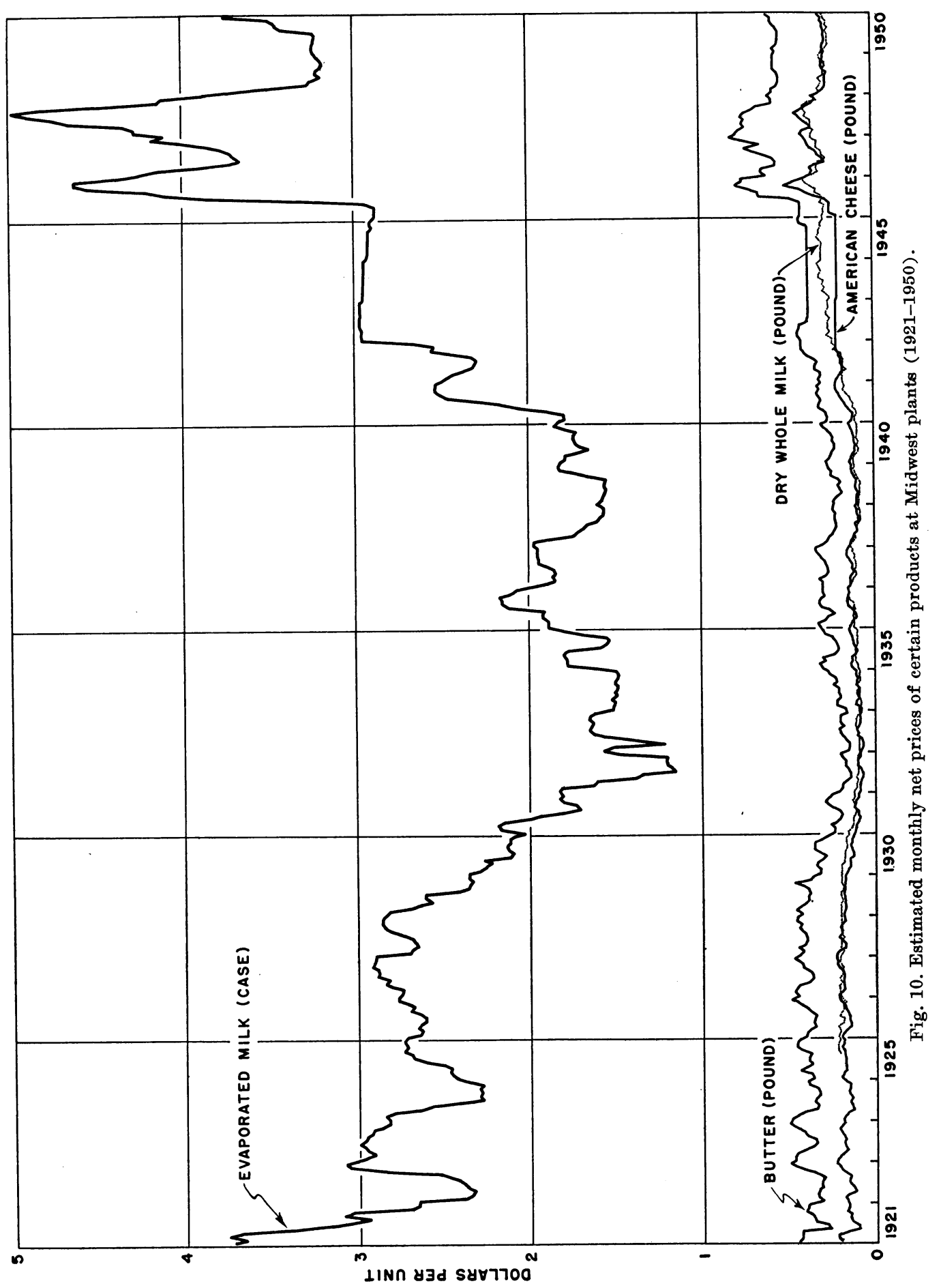


by the procedure. Suppose the demand in the eastern markets fell off during one month while the Chicago demand remained fairly stable. Suppliers might react too strongly in this situation and reduce shipments to the eastern markets (through shifting to Chicago) too rapidly, causing the price difference to increase while the percentage of receipts was going down. Obviously this could not continue, but the corrective reaction might err in the opposite direction during the next time period, giving inconsistent responses for each period.

From these comments, it can be seen that the use of percentages is not satisfactory. Use of absolute values of the shipments, with a knowledge of the actual supply areas and the level and shifts in demand at each market would be essential. Further, a full coverage of all markets would be required. Available information does not permit one to follow this course since it would require accurate estimation of all relevant demand and supply functions.

\section{Analysis of the Form Efficiency of the Manufactured Dairy Products Price Structure}

The first equilibrium price condition of section III outlines the basic argument upon which the present section is developed. All the simplifying conditions and assumptions that were given in reference to supply point $Y$ are essential for a concise presentation of the analysis. Without repeating these conditions, it should suffice to say that the analysis in this section is concerned with relative prices for the major products. In particular, the interest centers on examining the correspondence between actual relative net prices and those that would be expected under efficient conditions.

Four types of plant operations will be included in the set to be considered. All of these plants will utilize whole milk as their raw material. The types refer to the major products that are made: butter, American cheese, evaporated milk, and dry whole milk. Some by-product form usually will be produced in each case.

Evidence of relative price stability. As preliminary evidence of noticeable stability in the interproduct price relationships, figures 10 and 11 have been constructed. The first figure shows the manner in which the net prices of the four major products varied over the thirty-year period-a period with rather large differences in price level from one time to another. These net prices are estimates of actual values at Midwest plant locations and represent a subtraction of processing costs from the f.o.b. plant prices. The latter figure shows a set of seatter diagrams in which the net prices of evaporated milk, American cheese, and dry whole milk have been plotted against the simultaneous net prices of butter. A strong indication of a nearly constant ratio between the net prices is evident.

Since the influence of the value of by-products has not been included in the relationships shown by figure 11 and no independent data for comparative purposes have been presented to determine whether these actual relationships are efficient, a more detailed approach will include these two considerations. 

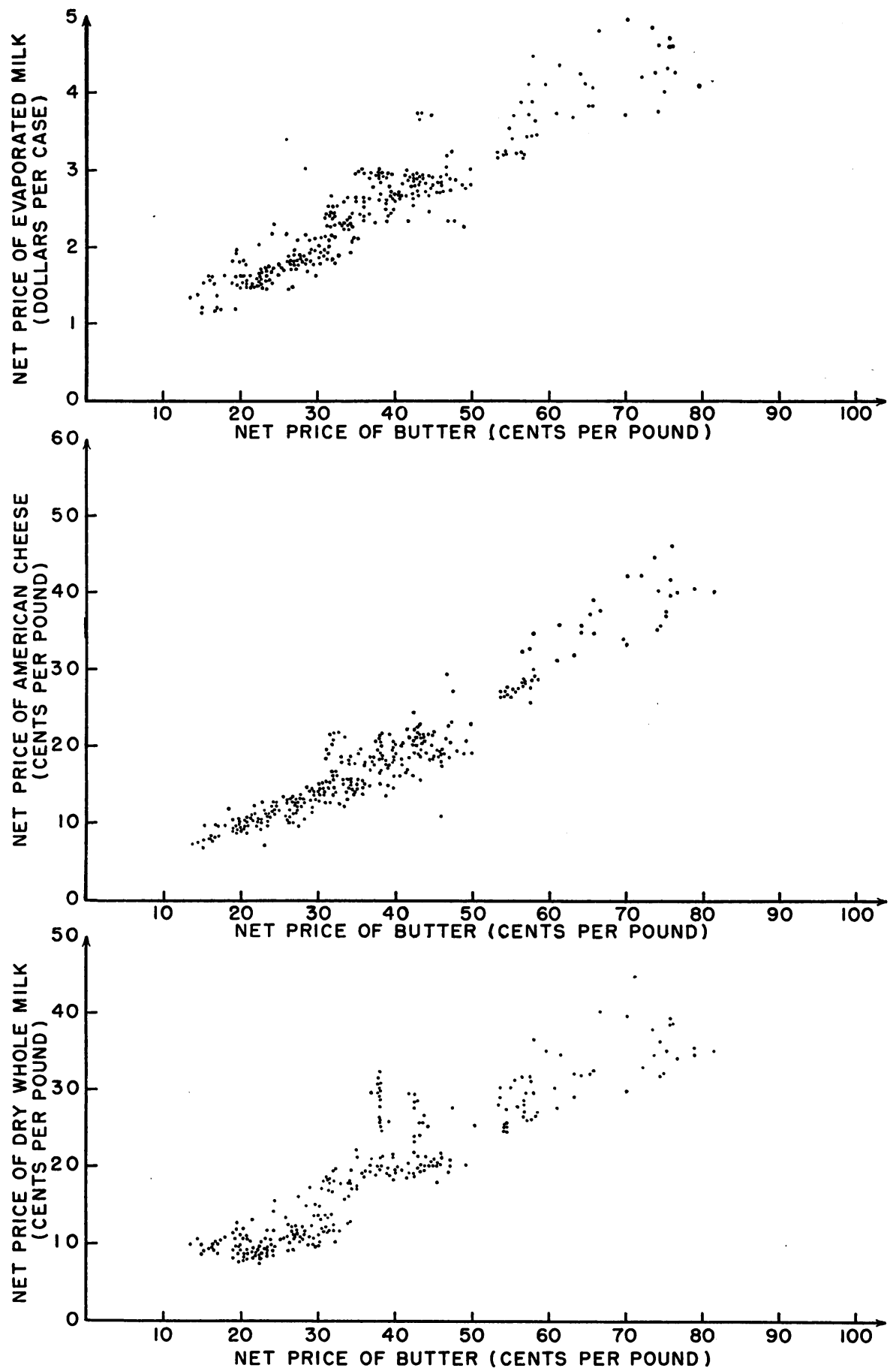

Fig. 11. Relationships between net prices of specified products at Midwest plants. 
Nature of plant operations. From the abstract discussion of the aspects of a productive activity in section II, it is evident that plant capacity has two dimensions-especially for plants that generate a continuous output flow. One dimension is the rate of operation and the second is the length of the day during which the rate continues. It will be assumed that the manufactured dairy products plants approximate continuous flow activities. The use of raw milk storage tanks facilitates the continuous overlapping of the quite discrete processes involved in each plant so that the packaged products evolve very soon after a plant starts a new day. In other words, the minimum time that can be used to define the rate of output is much less than an eighthour day, perhaps one hour.

Unless the period of daily operation is continuously variable, it is evident that a plant designed to be efficiently harmonious at a given rate will not be capable of assuming that rate unless the raw material flow to the plant is compatible with the attainable length of day. With milk production on farms exhibiting a marked seasonal variation, the optimum capacity plant must be designed around a compromise between an efficient rate size and an imperfectly conforming length of working day. The objective would be to have that plant which had the lowest weighted average unit cost for seasonal output. It will be assumed that all plants approach this objective. The costs used in this analysis will avoid the seasonal problem by being based on the weighted seasonal average concept.

To avoid the pitfalls of becoming too involved in minutiae, a simplified version of a plant's operation will be used instead of a detailed cost analysis, such as those being developed under other Regional studies at the present time. In all these plants, milk must be received, tested, and either fully or partially separated prior to being used in the production of final products. Logically, one may assign the costs involved in these activities to the units of raw milk used. Next, the separated components of the raw milk are used in discrete processes. The costs involved in each of these processes may be assigned to the units of the final products resulting from each process. Costs included in the two categories suggested in this paragraph do not contain the cost of the raw milk.

Two problems requiring solutions. The first problem to be solved is to establish the equilibrium relationships for the relative net prices of the major products. The second problem is to estimate the actual relationships from the real data and make comparisons. For the solution of the first problem, the following assumptions and definitions are essential:

\section{Assumptions}

a. All plants are isolocated, that is, similar to conditions at supply point $Y$ of section III. F.o.b. plant prices for each specific product are equal at all plants producing the product.

$b$. The aggregate cost involved in receiving, testing, and separating or standardizing is constant per 100 pounds of raw milk for all plants. This holds irrespective of the composition of the milk, scale of operation, or type of plant.

$c$. The unit processing cost per pound (per case for evaporated milk) of a given product, based on the supply prices of the services involved, is constant at all plants in which it may be produced. It is immaterial whether the product is a 
major output in one operation or a by-product in another operation. This means that processes within a plant are essentially independent and that change in the scale of a process is at constant cost. Independence in this case applies to the variable costs and to a constant proportionate sharing of fixed costs that are not unique to a given process. The scale implication here is interplant, that is, the scale of butter processing in a harmonized cheese plant might optimally differ from the scale in a butter-dry skim milk plant.

$d$. Cost elements in $(b)$ and $(c)$ include a "normal rate of return on investment." In other words, they are estimates of long-run costs.

$e$. No product inventories are held by plants, or, if held, then the rate of sale is equal to the rate of production.

$f$. Whole milk separation or standardization when fat must be removed is by means of cream with 40 per cent fat content at all plants.

g. Farmers have free choice in selling their milk and all milk is homogeneous in quality with respect to bacterial and other sanitary aspects but not to fat content.

\section{Definitions}

$a$. An operation means the production of a specific set of products of determinable quantities when portions of 100 pounds of original milk, having adjusted composition to satisfy composition requirements for the products, are operated on in a set of processes that make up the operation. A process may be thought of as buttermaking, evaporation, drying, et cetera, including in each instance packaging and associated activity.

$b$. Net value per 100 pounds of raw milk of a given composition means the difference between the gross value of the products which can be made from this milk in a given operation and the associated cost involved, excluding the cost of the milk. The values are computed f.o.b. plant, in dollar units. Let $R_{1}$ be the net value as defined above for operation $i$.

$c$. Let $M_{\mathrm{j}}=$ f.o.b. plant price minus processing cost in dollars per pound of the $j^{\text {th }}$ product. The case is the weight unit for evaporated milk. These $M$ 's are the net prices for the products.

d. Let $C=$ receiving, testing, and separating or standardizing cost in dollars per 100 pounds of original milk. This includes all costs involved in getting the milk ready for the processes.

$e$. Let $Q_{1 \mathrm{j}}=$ pounds of product $j$, which will result when 100 pounds of milk of a given composition are used in operation $i$. The case is the weight unit for evaporated milk.

It is evident from these assumptions and definitions that $R_{1}=\left\{\sum_{j} Q_{\text {ij }} M_{j}\right\}-C$ where $j$ covers those products resulting from utilizing milk in the $i^{\text {th }}$ operation. The equilibrium condition for this partial system of four operations is $R_{\mathrm{B}}=R_{\mathrm{E}}=R_{\mathrm{W}}=R_{\mathrm{C}}$ with the multiple equality being also equal to the price paid for the raw milk. Subscripts on the $R$ 's of the last sentence stand for butter operation, evaporated milk operation, dry whole milk operation, and American cheese operation, respectively. By means of the equilibrium condition and yield formulas to explicitly express the $Q$ 's as functions of the fat content of the raw milk, it is possible to establish the numerical value for the ratios of the net prices of the major products. The reason for limiting these ratios to the major products will be made clear later.

Physical yields. At this point a brief description will be given of the procedure employed in developing explicit product yield equations for milk used in each of the four operations. For details and a fuller discussion, the reader is urged to study Appendix A.

Milk is composed of fat, nonfat solids, and water. Disregarding certain minute quantities of chemicals which are added to the components of milk 
that are being processed, it is obvious that the final products must be compased of some combination of the basic parts of milk. In fact, government standards have been established to specify the minimum composition requirements for products moving in interstate trade. Practically all the manufactured dairy products do move between states and, from the economic point of view, it is to the advantage of a plant to come as near to these minimum requirements as possible, since consumers do not appear capable of detecting overminimum contents and fail to pay sufficient price premiums to cover higher product costs. Divergence is insignificant in those cases where state laws permit standardization of the raw milk prior to processing. Formulas will also be developed for unstandardized operations.

The calculation of yield formulas for products requires a knowledge of two sets of facts. First, one must know the composition requirements mentioned above. Second, one must know something about the quantitative relationship connecting the basic parts of raw milk. The former facts are readily available from government sources. The latter facts have been tentatively established and restudied by numerous researchers. The amount of divergence in these studies does not significantly affect the results of the present analysis. Consequently, only one result of the study on the relationship between fat and nonfat solids in raw milk is used. M. S. Jacobsen, in 1936, estimated the relationship as $Q_{\mathrm{N}}=7.07+.4 F$, where $Q_{\mathrm{N}}$ is the per cent of nonfat solids in milk having a fat content of $F$ per cent. These per cent values are in terms of weight. Since the original calculations for the present study were completed, the California Experiment Station (Jack, et al., 1951) has published a result, $Q_{\mathrm{N}}=7.07+.444 F$, for this relationship. The minor difference did not produce significant changes for a few trial recalculations so the original calculations were retained.

When one uses these sets of basic information in computing yield equations, the latter assume nonlinear forms. However, the divergence from linearity is very small for values of $F$ normally experienced. Because of this fact, the original yield formulas were rectified into linear forms which were more useful in subsequent calculation.

Net value equations. The net value equations can be written explicitly with the yield formulas substituted for the $Q$ 's. The subseripts on the $M$ 's identify the products, namely, b (butter), bu (dry buttermilk), n-s (dry skim milk), $n$ (bu $+n$-s as aggregated in the butter operation), e (evaporated milk), w (dry whole milk), c (American cheese), and wh (dry whey). It will be noted in the following equations that dry buttermilk has been aggregated with the dry skim milk in the butter operation as is the frequent practice. Further, this aggregate is assumed to be identical as a product to dry skim milk alone. For this reason $M_{\mathrm{n}}$ is equal to $M_{\mathrm{n}-\mathrm{s}}$ and the first symbol is used in place of the latter in the first equation of both the evaporated milk and dry whole milk operations. Whey butter is also treated the same as regular butter. The net value equations are as follows:

1. For all values of $F$

$$
\begin{aligned}
& R_{\mathrm{B}}=(1.2267 F-.1227) M_{\mathrm{b}}+(7.1703+.3975 F) M_{\mathrm{n}}-C \\
& \text { 2. For } F \leqq 3.765 \\
& R_{\mathrm{E}}=(.2916 F-.0243) M_{\mathrm{e}}+(7.3418-1.9500 F) M_{\mathrm{n}}-C
\end{aligned}
$$


For $F \geqq 3.765$

$R_{\mathrm{E}}=(.0342 F+.9448) M_{\mathrm{e}}+(1.0767 F-4.0538) M_{\mathrm{b}}+(.1257 F-.4733) M_{\mathrm{bu}}-C$

3. For $F \leqq 3.17$

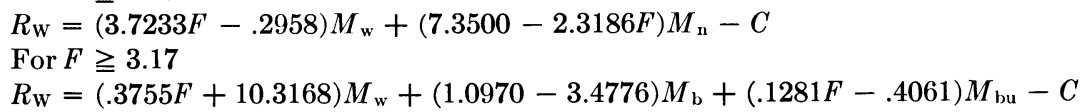

4. For all values of $F$

Standardized operation

$R_{\mathrm{C}}=(1.7157 F+2.2163) M_{\mathrm{c}}+(.5640 F-.9961) M_{\mathrm{b}}+(.0634 F-.1292) M_{\mathrm{bu}}$ $+(6.4868-.2149 F) M_{\mathrm{wh}}-C$

Unstandardized operation

$R_{\mathrm{C}}=(2.2556 F+1.400) M_{\mathrm{c}}+(.0887 F-.1083) M_{\mathrm{b}}+(6.1700-.1200 F) M_{\mathrm{wh}}-C$

The net value equation for the butter operation (as written to show the aggregation of buttermilk and skim milk) was taken as basic. For assigned values of $F$, the net value equations for the other operations were equated to this basic equation and $M_{\mathrm{e}}, M_{\mathrm{w}}$, and $M_{\mathrm{c}}$ were determined as functions of $M_{\mathrm{b}}, M_{\mathrm{n}}, M_{\mathrm{bu}}$, and $M_{\mathrm{wh}}$. The net price $M_{\mathrm{wh}}$ only entered into the function for $M_{\mathrm{c}}$. A separate solution was made for standardized and unstandardized cheese operations.

To clarify this point, the solutions for $F=4$ are as follows:

1. $M_{\mathrm{e}}=4.188 M_{\mathrm{b}}+8.096 M_{\mathrm{n}}-.028 M_{\mathrm{bu}}$

2. $M_{\mathrm{w}}=.328 M_{\mathrm{b}}+.741 M_{\mathrm{n}}-.010 M_{\mathrm{bu}}$

3. Standardized cheese operation:

$$
\begin{aligned}
& M_{\mathrm{c}}=.388 M_{\mathrm{b}}+.951 M_{\mathrm{n}}-.014 M_{\mathrm{bu}}-.620 M_{\mathrm{wh}} \\
& \text { Unstandardized cheese operation: } \\
& M_{\mathrm{c}}=.435 M_{\mathrm{b}}+.841 M_{\mathrm{n}}-.546 M_{\mathrm{wh}}
\end{aligned}
$$

The next step was the selection of a net price to be used as the base in specifying relative net prices for the other products. Butter was chosen for this purpose. Assuming $M_{\mathrm{b}}>0$, the above equations can be divided through by $M_{\mathrm{b}}$. Letting $r_{\mathrm{j}}=M_{\mathrm{j}} / M_{\mathrm{b}}$, the first equation above can be written as $r_{\mathrm{e}}=$ $4.188+8.096 r_{\mathrm{n}}-.028 r_{\mathrm{bu}}$. Similar expressions would result in the other cases and for other assigned values for $F$.

Figure 12 gives a graphic representation of these solutions for $F=3,4$, and 5. In the case of evaporated milk and dry whole milk, the three lines were nearly identical and could not be differentiated in plotting. To avoid a third dimension in the figure, $r_{\mathrm{bu}}$ and $r_{\mathrm{n}}$ were assumed equal. The physical similarity and usefulness of the two products make them nearly perfect substitutes. The assumptions given on the figure for $r_{\text {wh }}$ represent exaggerated maximum and potential minimum values which this variable could assume.

Peculiarities of cheese operations. A few comments are in order about the lines on figure 12 referring to the cheese operations (standardized and unstandardized). Considering the unstandardized operation with $r_{\mathrm{n}}$ less than approximately 0.20 , the line for $F$ equal to 3 is always below those drawn for higher values for $F$. This means that milk of low fat content has comparative advantage over milk of high fat content for the unstandardized operation. For values of $r_{\mathrm{n}}>0.20$, the order of advantage is reversed, if whey has a net price ratio near zero. However, past values experienced for $r_{\mathrm{n}}$ have been generally below 0.20 so the latter situation occurred very infrequently. 
Lines on figure 12 referring to the standardized cheese operation show two facts. First, under most situations of fixed values for $r_{\mathrm{n}}$ and $r_{\mathrm{wh}}$, the standardized operation has comparative advantage over the unstandardized operation regardless of milk composition. This is shown by the standardized

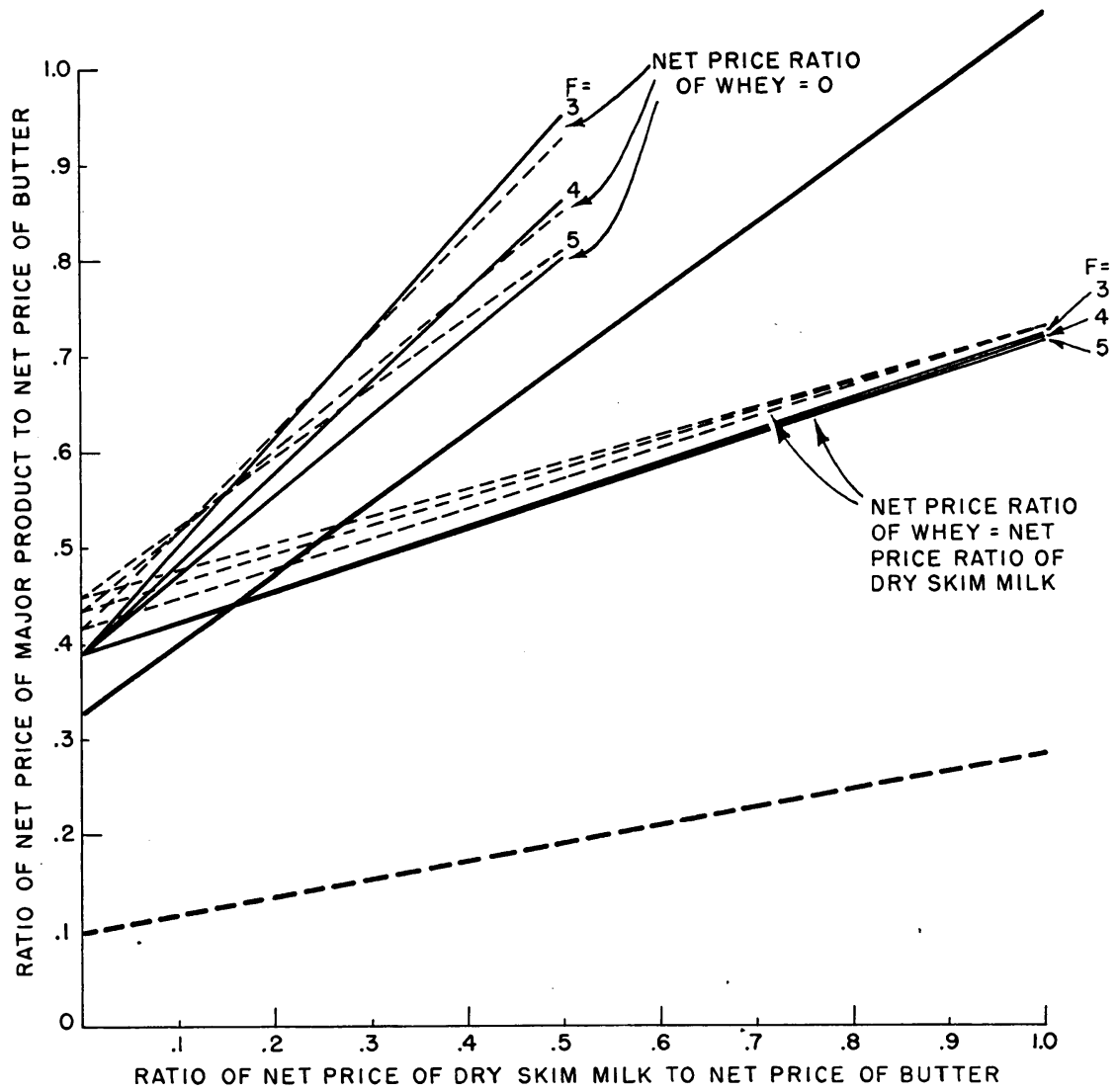

\footnotetext{
- - EVAPORATED MILK (BASED ON A POUND INSTEAD OF A CASE EQUAL 43.5 POUNDS)

- CHEESE (STANDARDIZED OPERATION)

- - - CHEESE (UNSTANDARDIZED OPERATION) DRY WHOLE MILK
}

Fig. 12. Equilibrium values of ratios of net prices of major products to the net price of butter under various assumptions about similar ratios of dry whey and dry skim milk.

lines falling below comparable unstandardized lines, indicating that the former operation could function at a lower equilibrium value for the net price of cheese relative to the net price of butter. Second, milk of high fat test has comparative advantage over milk of low fat test when used in a standardized operation. This is shown by the lines for $F$ equal to 3,4 , and 5 on the figure. As the fat test increases, the level of the line is lowered.

Additional assumptions required. From a study of the equations for $r_{\mathrm{e}}$, $r_{\mathrm{w}}$, and $r_{\mathrm{c}}$, it is obvious that no unique value as an equilibrium solution exists 
unless one can determine from other sources what the equilibrium values for $r_{\mathrm{n}}, r_{\mathrm{bu}}$, and $r_{\mathrm{wh}}$ should be. Immediately, without complete knowledge of all the demand intensities and supply functions for each product, this must be admitted an impossibility. This study does not supply such information and no other research analysis has done so. Due to shifts in the geographic location and magnitude of these demands relative to one another, no constant values for $r_{\mathrm{n}}, r_{\mathrm{bu}}$, and $r_{\mathrm{wh}}$ should be expected in dynamic equilibrium. The reason for the statement earlier in this section that the investigation would center on relative net prices for the major products is now apparent.

What can be done to remove the impasse presented in the last paragraph? Clearly, it requires some rational assumption about the equilibrium magnitudes of $r_{\mathrm{n}}, r_{\mathrm{bu}}$, and $r_{\mathrm{wh}}$. First of all, it will be assumed that milk utilization in the manufactured dairy products industry would require only minor shifts to reach equilibrium allocations. In other words, it is believed that the industry has been quantitatively quite near to equilibrium through time and that the demands for some of the products are quite inelastic in the neighborhood of the reigning price for each. Since the bulk of dry skim milk and dry buttermilk is a by-product of the butter operation, it is reasonable to believe that quantitative changes in the amount of milk used for butter will cause unidirectional changes in the prices for butter, dry skim milk, and dry buttermilk. (This statement refers to conditions in the real environment and not to the model, which is only partial. Buttermilk is usually incorporated with the skim milk in whole milk butter plants so the sources of dry buttermilk [whose value is usually less than that of dry skim milk] are the creamusing butter plants and butter production in other operations due to standardizing. The chain of shifts that are assumed for the disequilibrium case above is a simultaneous shifting of whole milk to other operations from butter plants and a shifting to whole milk utilization by some butter plants that previously utilized cream.) Consequently, a disequilibrium situation which would require a reduction in butter production would cause the full prices (and also net prices) of these three products to rise as equilibrium was approached. The effects of this general increase in the net prices of the three products on the values of $r_{\mathrm{n}}$ and $r_{\mathrm{bu}}$ are uncertain.

Dry skim milk and dry buttermilk are only partial uses of the primary liquid forms. Consequently, it is probably reasonable to assume that their net prices will not change much with the industry shift toward equilibrium. It follows that $r_{\mathrm{n}}$ and $r_{\mathrm{bu}}$ would decrease in this assumed case of overproduction of butter. Since the assumed case has been representative of the real case, as subsequent analysis will show, it is erring on the conservative side to make the following assumption, namely, equilibrium values for $r_{\mathrm{n}}$ and $r_{\mathrm{bu}}$ at each point in time will be assumed equal to their values as estimated from actual price and cost data. During some of the early years when dry buttermilk prices were unavailable, $r_{\mathrm{bu}}$ will be assumed equal to $r_{\mathrm{n}}$ and the common value will be estimated with the use of the available dry skim milk prices.

The problem of a decision about the equilibrium value of $r_{\mathrm{wh}}$ remains. From past history it appears that liquid whey was customarily returned to farmers for farm feed or discarded as waste. Even from recent price and processing cost data, it is evident that the net price for dry whey has always 
been near zero. In accord with these observations, it is apropos to assume that $r_{\mathrm{wh}}$ would have been zero under past equilibria for the industry. An upper limit assumption of $r_{\mathrm{wh}}=1 / 2 r_{\mathrm{n}}$ will also be used. The latter has little to support it but might have been relevant during time periods when $r_{\mathrm{n}}$ was unusually low.

The geographic setting and relative net prices. Only one more obstacle exists before the detailed method of estimating equilibrium relative net prices for the major products, for comparisons with actual values, can be used. Simply stated, a real geographic area in the United States must be found where the location relative to demand outlets is such as to make equally feasible from the economic point of view the production of butter, evaporated milk, dry whole milk, or American cheese. The area must be large enough so that marginal utilization shifts need not progress to a full single use before equilibrium can be restored for the entire industry. Changing utilization in other areas of the country would limit the amount of shifting required in the selected area.

From information on location of plants by types, it has been concluded that the four-state area of Wisconsin, Illinois, Iowa, and Minnesota represents a multipurpose area quite well. Figure 13 shows the areas of densest production by type of product in this four-state area. The dry whole milk plants, although not shown, are located principally in the butter-producing area. Actually, a region encompassed by a circle with a radius of about 150 miles and centering on the northeast corner of Iowa would be more representative than the total area. The latter will be assumed to satisfy the requirements designated in the last paragraph. Plants within this area must be assumed to have equal f.o.b. plant prices for products by types and equal processing costs in order to have equal net prices. Having equal f.o.b. plant prices is equivalent to a situation of no locational advantage with respect to selling points. Since this area actually supplies products to far distant markets in the United States and relatively flat zonal transportation rates exist for such shipments, it is likely that differences in plant prices for products are negligible. (Flat zonal rates to distant markets augment the area over which multiutilization of raw milk is economically feasible with equal advantage in all uses. Locational advantages due to weight of final products are reduced and spread out geographically. Further, the location of the many distant markets in diverse directions from a localized supply area tends to obliterate the locational advantage of producing specific products as is so commonly depicted in location theory with reference to one or a few markets.) Finally, the homogeneous nature of opportunities within the area supports an assumption of equal processing costs for each product at all plants.

Prior to 1951, Wisconsin prohibited the standardization of milk for the production of cheese. Consequently, the net value equation for the unstandardized cheese operation will be used in the analysis of relative net prices for the major products. From figure 12 it is evident that the unstandardized operation is at a comparative disadvantage to the standardized operation. Also, with $r_{\mathrm{wh}}$ near zero and $r_{\mathrm{n}}<0.20$, which were the common actual values during the 1921-1950 period, the unstandardized cheese operations with milk of low fat content had comparative advantages in competition with 
plants producing other products. Data on fat tests of receipts of milk at various plants in Wisconsin indicate a positive reaction by cheese plants to this condition, since the fat test of cheese plant receipts has been consistently lower than in other uses (Wisconsin State Department of Agriculture, 1945).
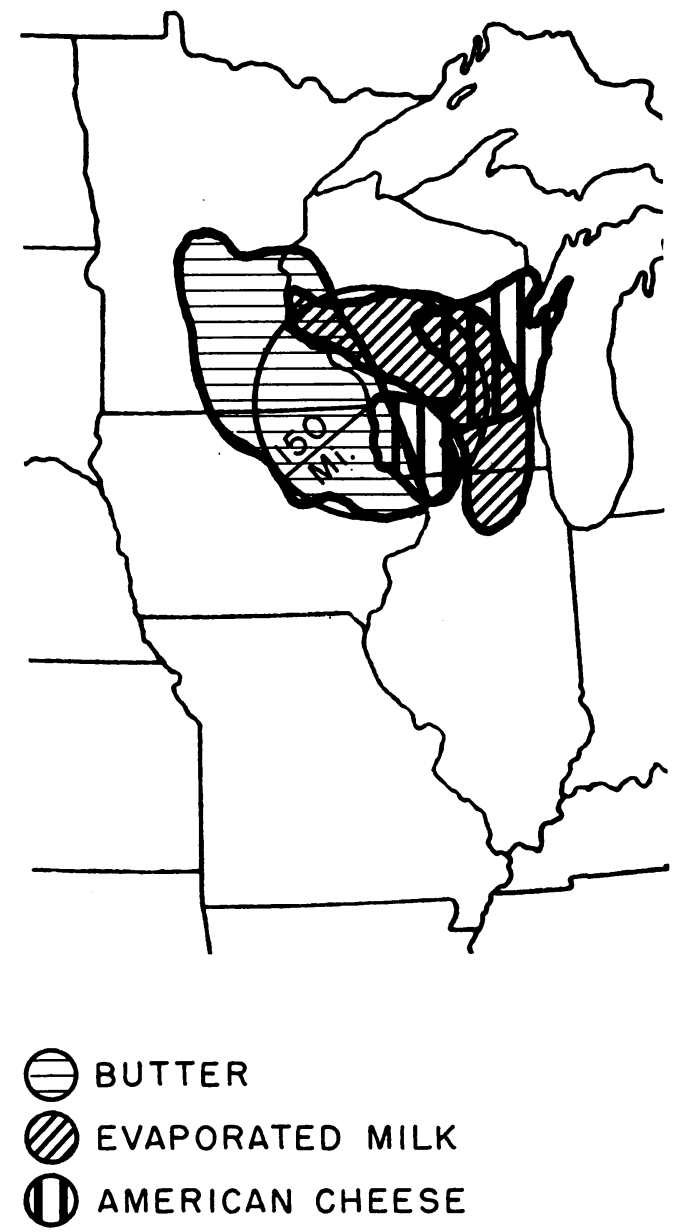

Fig. 13. Midwest areas of dense production by product type.

Figure 12 indicates that the fat tests of milk entering other types of plants present no particular advantages in use, provided the plants are organized harmoniously in accord with whatever average fat test they are likely to have. In order to key the cheese operation into such a setting, it is necessary to assume that the actual average fat test of milk used by cheese plants has been reduced to a stable operational level, which would remain unchanged by movements of the industry toward an equilibrium utilization of milk. The analysis that follows is based on monthly time intervals, so the assigned values of $F$ must vary over the season. Following the above argument, it is 
reasonable to have these assigned values of $F$ equal to those experienced at American cheese plants. The following schedule for values of $F$ was used.

Value of $F$
3.6
3.7
3.8
3.9

Months of year

April, May, June, and July

February, March, and August

January, September, and December

October and November

Summary. The calculations of the equilibrium ratios of the net prices of evaporated milk, dry whole milk, and American cheese relative to the net price of butter were made as listed below. The steps are as follows and apply for each month.

1. For each assigned value of $F$, equations for $r_{\mathrm{e}}, r_{\mathrm{w}}, r_{\mathrm{c}}$ were derived by imposing the equilibrium condition of equal net value per 100 pounds of milk in all uses and through division by $M_{\mathrm{b}}$ as discussed previously. The net value equation for the butter operation was used as the base.

2. Using the net prices for butter, dry buttermilk, and dry skim milk as calculated for the midwest, an estimate was made for $r_{\mathrm{n}}$ and $r_{\mathrm{bu}}$ to be substituted into equations given in step 1. Direct assumptions were made for $r_{\mathrm{wh}}$ as shown in figure 14 .

3. As lower limits for $r_{\mathrm{e}}, r_{\mathrm{w}}$, and $r_{\mathrm{c}}$ (lines $D$ in panels I and II, $C$ in panel III), values were also calculated under the assumption that all skim products had zero net prices.

To complete the computational discussion, the method of estimating the actual f.o.b. plant ratios of the net prices of the products relative to the net price of butter will be given. The actual ratios for the major products relative to butter are shown in figure 14 as line $A$ of each panel. For all products other than butter and cheese, the prices as compiled from government reports were taken as f.o.b. plant prices. The prices for dry products are United States averages. For evaporated milk, the East North Central average prices were used for the area specified in this analysis. The net prices of the products in this category were estimated by subtracting the processing cost as given in Appendix B. In the case of butter and cheese, the Chicago price was chosen as a base. From these Chicago prices were subtracted the freight, handling, and storage allowances (Appendix B) in order to estimate f.o.b. plant prices. Finally, the relevant processing cost estimates were deducted to arrive at the net prices for butter and cheese. Once the net prices f.o.b. plant had been estimated, the calculation of their ratios to the net price of butter was simply a matter of division. It should be noted that one can compute equilibrium and actual net price ratios for each possible pairing of major products. These are of use in ranking the positions of advantage for producing these major products at a given point in time. This will be given later.

Before interpreting the relationships between the actual and equilibrium net price ratios as shown in figure 14, a brief explanation should be given of the method employed in estimating the historical series of specific processing costs. These costs are the monetary estimates of the long-run real costs involved in the processing activities. Since no continuous series of plant studies have been made for this purpose, an alternative procedure was used. The basic approach was to utilize a set of costs for the year 1943 and, with reasonable price indexes, to move the components of these costs to make estimates for other years. Only annual estimates were made and applied for each month 


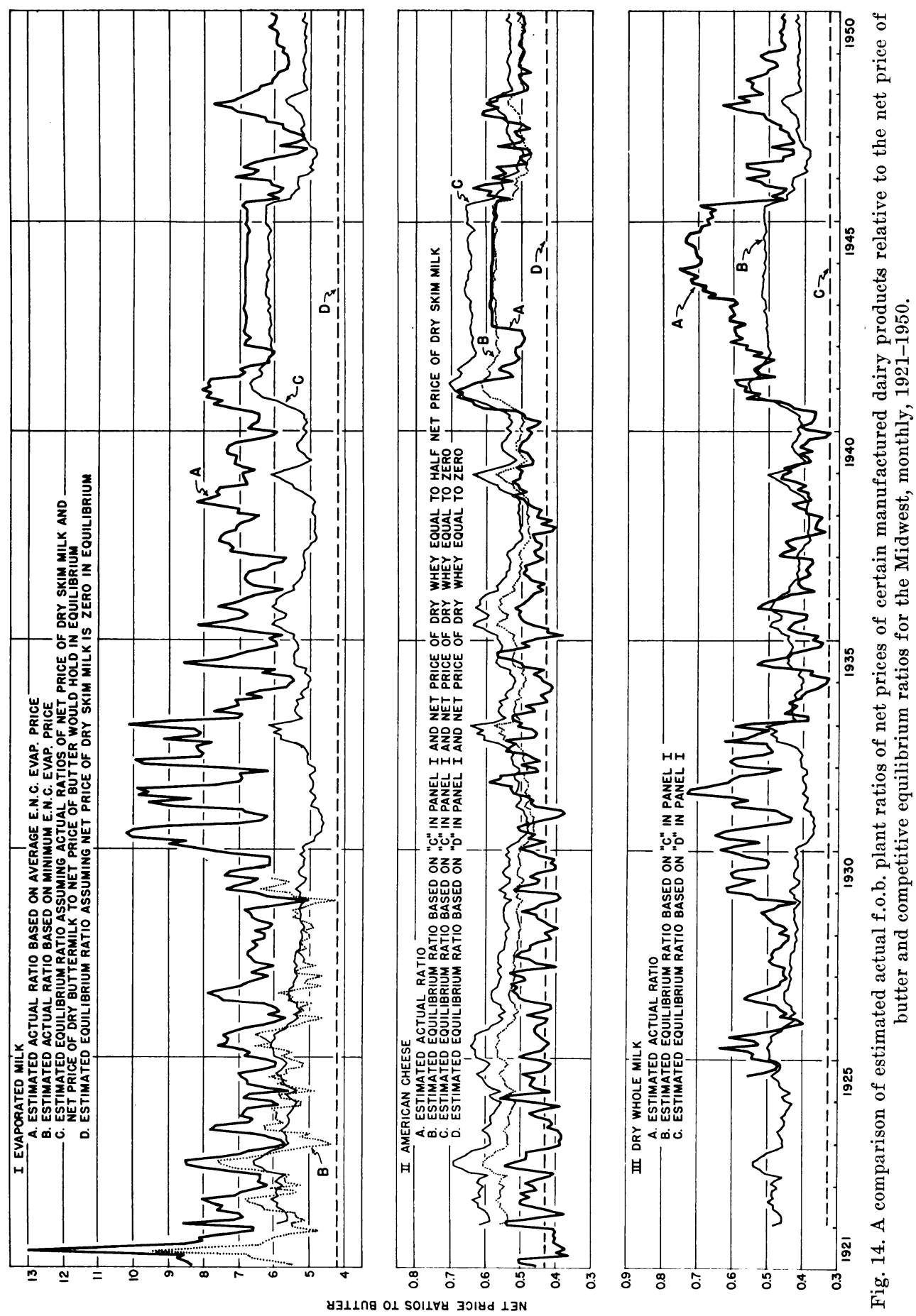


of the year. For fuller details on the estimates, the reader is referred to Appendix B.

Interpretation of results. What can one deduce from the results of this detailed analysis of relative prices? Although the problem is an integral consideration, the discussion will proceed product by product. Figure 14 should prove to be a helpful visual aid in following the arguments.

If the average East North Central evaporated milk price is accepted as equivalent to the f.o.b. plant price for national brands, a continuous condition of overpricing relative to butter appears to have existed. Except for a few time periods (1930-1933 and 1943-1945) in which dry whole milk prices were exceptionally high, evaporated milk prices for national brands were competitively too high relative to cheese and dry whole milk as well as butter. However, if one again accepts the minimum East North Central evaporated milk price as equivalent to the f.o.b. plant price for local brands, in this case a situation of competitive relative prices with respect to butter seems to have prevailed. This judgment is founded on the somewhat erratic fashion by which line $B$ follows line $C$ in panel I of figure 14 for the years for which the data were available. These conclusions and others which follow are based on the assumption that the paying schedule for milk was equal or approximately so for all plants. This assumption will be partially tested in section VII for a few of the prewar and postwar years. Much more could be inferred from the evidence to support discussion on particular historical conditions for evaporated milk in the thirty-year period, but these topics would represent only special situations of the two main conclusions above.

Proceeding to American cheese and panel II of figure 14, the results appear to place this product at a disadvantage with respect to a butter-dry skim milk operation, that is, the price of cheese apparently was consistently too low with respect to the price of butter. There is some evidence, however, that part of the processing costs utilized in this study are not always borne by the cheese plant, so that the actual net price ratio of cheese to butter could be raised about 0.02 units throughout the period. The rather common practice of assemblers supplying boxes to plants, either free or at a reduced price, is responsible for such an adjustment. Even with such an adjustment, cheese appears to have been underpriced relative to butter.

A further consideration related to the level of the equilibrium ratio (line $C$ of panel II) might place butter and cheese in an efficiently competitive price ratio. First of all, the specification of 37 per cent (the legal maximum is 39 per cent) moisture content as a basis for the yield equations might be a little too stringent. However, even though there is evidence to support the 37 per cent figure, the available prices for cheese do not reflect the moisture premium usually paid for cheese having a lower moisture content than 39 per cent. By permitting the moisture content to rise 2 per cent, the equilibrium ratio could be lowered about 0.02 units. This adjustment in conjunction with a comparable one suggested in the last paragraph would reposition lines $A$ and $C$ in such a way as to place them on approximately the same level since 1940.

Second, prior to 1940 it was probably true that a butter-dry skim milk operation could secure a higher net value from whole milk than could a cheese 
operation. However, the number of butter plants which received whole milk and which were in a position to utilize the skim milk in drying (either by internal use or by sale to a drying plant) was probably quite small. The normal marginal use for the skim milk was to return it to the farmer for feed. Where farm use possibilities were low, the rated value of the skim milk by farmers also was low. Under such circumstances the net value of skim milk would be considerably lower than that estimated by a dry skim milk utilization. These two net values were kept from equality by the high risk and uncertainty connected with entry into the drying field. This makes the assumption of all skim uses being in equilibrium valuewise invalid in the real environment. It was not until World War II and indirect governmental subsidization and encouragement that the risk and uncertainty of the venture were reduced. Consequently, it seems reasonable that a much lower average net value should have been employed prior to 1940. If this lower net value for skim milk was transformed to the dry skim product, it probably would have resulted in a net price ratio to butter ranging between zero and 0.05 instead of 0.10 to 0.20 as was used. With the previous moisture adjustment, this change would place the equilibrium ratio between 0.400 and 0.500 . The raising of the actual ratio in accord with the argument about box supplies would place this ratio in a similar position. These adjustments are believed to be valid modifications of the original basic computations. The sporadic importance and size of these adjustment values made it impossible to incorporate them into the basic calculations. Cheese and butter prices have probably conformed quite closely to competitive relative net prices.

Before discussing dry whole milk, further implications of the overvaluation of skim milk should be pointed out. By overvaluing skim milk, the previous conclusions with respect to evaporated milk are strengthened since it makes the equilibrium ratio higher than it should be. The same effect applies in the case of dry whole milk.

In panel III of figure 14, dry whole milk prices appear to have been fairly competitive relative to butter during the early period of the late 1920's and from 1934 to late 1942. From 1930 through mid-1933, the net price for this product was approximately 1.4 times the equilibrium value with respect to the net price for butter. From late 1942 to the middle of 1944, the relative net price rose from an equilibrium level to about 1.6 times the equilibrium value and dropped back to an equilibrium level by decontrol of prices in 1946. During the postwar period, dry whole milk has continued to exhibit higher than competitive prices with respect to butter.

Advantages and disadvantages. Considering all operations simultaneously, the following aggregated information is presented on the ranking of potential net values for milk used in the four operations. For the 305 months (August 1925 through December 1950), the number of times each operation assumed a given rank, irrespective of magnitude, is given in table 11. These rankings refer to the order of value for milk used in each operation with the value based on product prices minus processing costs.

The aggregated rankings are in conformity with the previous conclusions. The dominant number of times the evaporated milk operation ranked first should be noted, with the dry whole milk operation usually taking first place 
in those cases when the former ranked second or third. It should also be evident that the adjustments suggested for cheese and skim milk would not greatly change the rankings of the evaporated or dry whole milk operations. The effect of these changes would mostly equalize the number of times the butter and cheese operations assumed the second, third, and fourth rankings-say, at 40,120 , and 144 , respectively.

In summary, the relative prices of national brand evaporated milk appear to have been consistently too high. Dry whole milk relative prices have exhibited discontinuous periods of being too high with a portion of this time under intentional World War II incentive pricing for the product. Butter and American cheese prices, with rational adjustments in the model, have

TABLE 11

NUMBER OF TIMES DESIGNATED OPERATIONS ASSUMED GIVEN RANKS IN COMPARISONS OF NET VALUES PER 100 POUNDS MILK, MIDWEST AUGUST 1925-DECEMBER 1950

\begin{tabular}{|c|c|c|c|c|}
\hline Rank & $\begin{array}{c}\text { Evaporated } \\
\text { milk } \\
\text { operation }\end{array}$ & $\begin{array}{c}\text { Dry } \\
\text { whole milk } \\
\text { operation }\end{array}$ & $\begin{array}{c}\text { Butter } \\
\text { operation }\end{array}$ & $\begin{array}{c}\text { American } \\
\text { cheese } \\
\text { operation }\end{array}$ \\
\hline First. & 213 & 90 & 1 & 1 \\
\hline Second. & 88 & 134 & 78 & 5 \\
\hline Third. . & 4 & 59 & 200 & 42 \\
\hline Fourth . . . . . . . . & 0 & 22 & 26 & 257 \\
\hline
\end{tabular}

been competitive in their relative values, but, in general, both have had prices too low relative to evaporated milk and dry whole milk. It is not believed that either the inclusion of potential discounts or premiums not reflected in quoted prices or a relaxing of the assumption that plant sales are from current production and not speculative stocks would alter these conclusions.

Utilization shifts and comparative advantage. Before completing the analysis of relative prices, a partial investigation will be presented to assess how efficiently utilization shifts have been in response to the relative price distortions. To facilitate the discussion, table 12 has been constructed. Since much of the butter production was from plants using cream instead of whole milk, the left portion of the table could be misleading from the viewpoint of whole milk utilization. For the reduced number of operations, the right portion of the table is probably more relevant.

From these percentage figures, the production of dry whole milk appears to have been responsive to price advantages. Utilization of milk for butter production has generally declined in percentage terms with a low relative price. Evaporated milk utilization does not seem to have increased sufficiently in response to a continuously high relative price. The situation for American cheese violates what should have been expected. Part of the discrepancy is removed upon considering the right portion of the table where the percentage figures have remained approximately stable since 1929 and were declining prior to that.

This partial inconsistency in long-period utilization shifts for milk used in producing cheese exposes two things. First, the previously suggested ad- 
justments for cheese in the relative price analysis were probably valid. Second, the use of percentages can be extremely misleading when no account can be made for demand shifts. With inadequate knowledge of product demands and their shifts, the use of absolute values is thwarted.

TABLE 12

PERCENTAGE UTILIZATION OF MANUFACTURING GRADE MILK BY SUBGROUPS, UNITED STATES, 1924-1950

\begin{tabular}{|c|c|c|c|c|c|c|c|}
\hline \multirow{2}{*}{ Year } & \multicolumn{4}{|c|}{ Within four uses } & \multicolumn{3}{|c|}{ Within three uses } \\
\hline & Butter & $\begin{array}{c}\text { American } \\
\text { cheese }\end{array}$ & $\underset{\text { milk }}{\text { Evaporated }}$ & $\begin{array}{c}\text { Dry } \\
\text { whole milk }\end{array}$ & $\begin{array}{c}\text { American } \\
\text { cheese }\end{array}$ & $\underset{\text { milk }}{\text { Evaporated }}$ & $\begin{array}{c}\text { Dry } \\
\text { whole milk }\end{array}$ \\
\hline 1924. & 79.6 & 13.1 & 7.0 & 0.3 & 64.2 & 34.3 & 1.5 \\
\hline 1925. & 79.2 & 13.6 & 6.9 & .3 & -65.4 & 33.2 & 1.4 \\
\hline 1926 . & 80.7 & 12.5 & 6.6 & .2 & 64.8 & 34.2 & 1.0 \\
\hline 1927. & 80.9 & 11.7 & 7.1 & .3 & 61.3 & 37.2 & 1.5 \\
\hline 1928. & 79.7 & 12.6 & 7.4 & .3 & 62.1 & 36.5 & 1.4 \\
\hline 1929. & 81.8 & 9.6 & 8.3 & .3 & 52.8 & 45.6 & 1.6 \\
\hline 1930. & 81.9 & 10.0 & 7.9 & .2 & 55.2 & 43.6 & 1.2 \\
\hline $1931 \ldots$ & 82.8 & 9.4 & 7.6 & .2 & 54.7 & 44.2 & 1.1 \\
\hline $1932 \ldots$ & 82.2 & 9.3 & 8.2 & .3 & 52.2 & 46.1 & 1.7 \\
\hline 1933. & 81.6 & 9.7 & 8.4 & .3 & 52.7 & 45.7 & 1.6 \\
\hline $1934 \ldots$ & 80.5 & 10.6 & 8.6 & .3 & 54.4 & 44.1 & 1.5 \\
\hline $1935 \ldots$ & 78.5 & 11.5 & 9.5 & .5 & 53.5 & 44.2 & 2.3 \\
\hline $1936 \ldots \ldots \ldots \ldots \ldots$ & 77.5 & 11.8 & 10.5 & .2 & 52.4 & 46.7 & .9 \\
\hline $1937 \ldots \ldots \ldots \ldots \ldots$ & 77.9 & 12.1 & 9.8 & .2 & 54.8 & 44.3 & .9 \\
\hline $1938 \ldots$ & 77.4 & 12.5 & 9.6 & .5 & 55.3 & 42.5 & 2.2 \\
\hline $1939 \ldots$ & 77.6 & 11.9 & 10.0 & .5 & 53.1 & 44.6 & 2.3 \\
\hline $1940 \ldots$ & 76.1 & 12.5 & 10.9 & .5 & 52.3 & 45.6 & 2.1 \\
\hline $1941 \ldots$ & 71.2 & 14.6 & 13.5 & .7 & 50.7 & 46.9 & 2.4 \\
\hline $1942 \ldots$ & 67.0 & 17.6 & 14.4 & 1.0 & 53.3 & 43.6 & 3.1 \\
\hline $1943 \ldots$ & 68.6 & 15.7 & 13.5 & 2.2 & 50.0 & 43.0 & 7.0 \\
\hline $1944 \ldots$ & 64.0 & 17.4 & 15.8 & 2.8 & 48.3 & 43.9 & 7.8 \\
\hline $1945 \ldots$ & 59.6 & 19.2 & 17.6 & 3.6 & 47.5 & 43.6 & 8.9 \\
\hline $1946 \ldots$ & 59.1 & 20.4 & 16.7 & 3.8 & 49.9 & 40.8 & 9.3 \\
\hline $1947 \ldots$ & 60.2 & 21.3 & 15.7 & 2.8 & 53.5 & 39.5 & 7.0 \\
\hline $1948 \ldots$ & 58.6 & 20.9 & 17.4 & 3.1 & 50.5 & 42.0 & 7.5 \\
\hline $1949 \ldots$ & 63.6 & 21.1 & 13.2 & 2.1 & 58.0 & 36.3 & 5.7 \\
\hline $1950 \ldots \ldots \ldots \ldots \ldots \ldots$ & 63.6 & 20.2 & 14.0 & 2.2 & 55.5 & 38.4 & 6.0 \\
\hline
\end{tabular}

Source: Computed from data given in 1952 Agricultural Outlook Charts, U. S. Bur. Agr. Econ., Wash., D.C. Oct., 1951 .

\section{Analysis of the Intraseasonal Temporal Efficiency of the Manufactured Dairy Products Price Structure}

At the outset of this section the reader should be cautioned not to expect a very rigorous or detailed investigation of the intraseasonal temporal aspect of the price structure. Inadequate data on storage costs, the time elapsing between the movement of a given lot into and out of storage, and the level and shifts in the demand for each product over time preclude such an intensive study.

Long-period and short-period points of view. It should be pointed out that both the geographic and relative price analyses that have been made represent considerations through time. From the long-period point of view, these analyses show a rather efficient movement of the space and form 
aspects of pricing when one realizes that relatively large changes in the underlying conditions have occurred with the passage of time. In general, one could conclude that the industry has been quite efficient in the allocation of the milk to the various uses and the distribution of products to consuming centers. Temporal adjustments and resulting prices have been reasonably consistent in these respects. In the present section, interest centers on the within season price variation for products and indirectly for milk. It is evident that this pricing aspect is important, since the analyses of space and form aspects do not consider the problem of whether the month-to-month movements of prices are consistent with the costs involved in those storage activities which are economically feasible.

Dual price effect of storage operations. In the discussion of the third equilibrium price condition for the simplified model in section III, a quite thorough coverage of the problem of price variation over time was given. It is apparent that a complete investigation would be exceedingly complex. The full burden of future uncertainty bears on the problem, since the volumes of the products that are placed in storage have the dual role of determining (in conjunction with current production and demands at the relevant time points) both the level of current prices and the level of prices at a later time when withdrawal takes place.

Implicit in the previous discussion on temporal equilibrium for price variations was the assumption that volumes marketed throughout a season could be adjusted through storage operations in such manner as to have price variations just adequate in amount to cover such storage operations. Under static demand conditions, in a schedule sense, which permit price movements along the schedules without generating shifts in the same, the pattern of storage and marketings would be relatively simple provided that persons involved accurately anticipated this stable condition and its quantitative aspects. In view of the relatively small amount of price variation required to cover storage costs, the flow of products to consumers would be nearly constant over the season, with storage operations merely leveling out the fluctuating seasonal flow of production. To cover storage costs, suppliers would permit their offer price to drop slightly during the flush period of production in order to induce the demand to accept slightly more than the seasonal average production flow. The remainder, which would be stored, would permit them to raise their offer price in the deficit period of production sufficiently to cover the storage costs over the interval of time. This degree of price variation would probably be insufficient to produce a schedule shift in demand for each product over the season, that is, the slowness and small size of the price variation would not have irreversible impact on tastes.

Interaction between consumer tastes and price changes. However, under conditions of rapidly increasing or decreasing shifts in demand over the season, due to unidirectional shifts in tastes or in disposable, personal money income, the interdependence of price variation and these demand shifts may be quite large. For instance, with a falling demand anticipated correctly, storage would be expected to have been started later than usual in the flush period and in smaller volumes. This competitive restriction on volumes entering storage would be accompanied by a flexible decline in price up to the time 
when price had been depressed sufficiently to make storage feasible. The declining price could very easily have a retarding influence on the regular shifting of the demand for the product caused by external forces. During the deficit period of production the meager storage supplies would have a pricestrengthening influence in the face of falling demand and may tend to augment the rapidity of the decline in demand. The competitive balancing of these influences on the regular decline in demand, to result in price variation to cover storage costs, could operate to the detriment of the industry in the succeeding season, since tastes could have been altered in a detrimental way by the price movements.

Two objectives of the temporal analysis. The subsequent analysis of seasonal price variation will be aimed at ascertaining two facts: (1) have actual price variations been compatible with an estimate of storage costs? and (2) has the pattern of storage during years of increasing and decreasing prices been compatible with a competitive reaction to correct expectations? Only four products will be considered, namely, butter, American cheese, evaporated milk, and dry whole milk.

In answering the first question a rather broad point of view must be taken since it is impossible to ascertain from available data the exact time lapse between into and out of storage movements for particular volumes, which would be necessary if average total storage costs were not constant per unit of p:oduct per unit of time. An average time lapse of six months will be assumed, beginning in June and ending in December. Further, it will be assumed that marginal storage costs per unit of product per unit of time are constant and that cost components not associated with time are negligible, so the price variation during the storage period will be approximately a linear increase. Evidence on actual storage operations indicates that the storage period begins in April or May and lasts until February or March of the following season. Consequently, the June-December interval should be valid for a "within storage period" analysis of price variation. Table 13 shows the price increases for the June-December interval for all years except the 1942-1946 controlled period.

Before evaluating these figures, a few crude estimates of the average total unit storage costs for six months will be made. They are based on information given by members of the trade. Including about a point drop in the score rating of butter in storage, the following estimates for this product appear to be reasonable: ( $a$ ) 3 cents per pound during the 1920's ; $(b) 2$ cents per pound during the 1930's, and 1940 and 1941 ; and $(c) 4$ cents per pound during 1947 through 1950. Comparable costs for evaporated milk are 23, 15, and 30 cents per case ; for dry whole milk, 1.5, 1, and 2 cents per pound; and for cheese about $2,1.3$, and 2.3 cents per pound. These estimates, if valid, indicate that butter has the smallest storage cost per unit of milk equivalent.

Interpretation of results. Returning to the information given in table 13, a few general conclusions can be reached. Considering the years 1929, 1930, 1938, and 1948, which were periods of rapid decline in prices due to falling demands and stable supplies, it would appear that inaccurate expectations existed in anticipation of the trend in demands. Storage operations were too heavy and losses resulted. In 1933 optimistic expectations, possibly based on 
a belief that government fiscal policy would relieve the depression, led to heavy storage during the summer and losses during the following winter. The opposite situation is apparent in years like 1922, 1926, and 1947 when demand increased throughout the season.

In reference to price variations during other years there is evidence that butter storage operations did not progress far enough to reduce price varia-

TABLE 13

AMOUNTS BY WHICH THE DECEMBER PRICE EXCEEDED THE JUNE PRICE FOR DESIGNATED PRODUCTS, 1921-1941 AND 1947-1950

\begin{tabular}{|c|c|c|c|c|}
\hline \multirow[t]{2}{*}{ Year } & Butter* & $\begin{array}{c}\text { American } \\
\text { cheese } \dagger\end{array}$ & $\begin{array}{c}\text { Dry } \\
\text { whole milk } \ddagger\end{array}$ & \multirow{2}{*}{$\begin{array}{c}\begin{array}{c}\text { Evaporated } \\
\text { milk }\end{array} \\
\text { cents per case }\end{array}$} \\
\hline & \multicolumn{3}{|c|}{ cents per pound } & \\
\hline$\ldots \ldots \ldots \ldots \ldots \ldots$ & 13.8 & 5.9 & $\S$ & -45 \\
\hline $1922 \ldots \ldots \ldots \ldots \ldots \ldots \ldots$ & 18.7 & 10.2 & $\S$ & 66 \\
\hline $1923 \ldots \ldots \ldots \ldots \ldots \ldots \ldots \ldots$ & 13.0 & .9 & $\S$ & -8 \\
\hline $1924 \ldots \ldots \ldots \ldots \ldots \ldots \ldots \ldots$ & 5.5 & 3.3 & $\S$ & -1 \\
\hline $1925 \ldots \ldots \ldots \ldots \ldots \ldots \ldots \ldots$ & 6.7 & 1.7 & $\S$ & 11 \\
\hline$\ldots \ldots \ldots \ldots \ldots$ & 13.1 & 5.6 & 1.1 & 8 \\
\hline$\ldots \ldots \ldots \ldots$ & 9.0 & 4.7 & 0 & 9 \\
\hline$\ldots \ldots \ldots \ldots$ & 5.7 & .3 & .8 & 16 \\
\hline $1929 \ldots$ & -2.8 & .2 & -.1 & -24 \\
\hline $1930 \ldots$ & -3.2 & -1.9 & -1.4 & -2 \\
\hline $1931 \ldots \ldots \ldots \ldots \ldots \ldots \ldots$ & 6.8 & 1.1 & .4 & -15 \\
\hline $1932 \ldots \ldots \ldots \ldots \ldots \ldots \ldots \ldots$ & 5.6 & 1.6 & -.6 & 12 \\
\hline$\ldots \ldots \ldots \ldots \ldots$ & -3.2 & -2.2 & -.3 & 4 \\
\hline$\ldots \ldots \ldots \ldots \ldots$ & 6.3 & 1.7 & .4 & -1 \\
\hline$\ldots \ldots \ldots \ldots \ldots$ & 7.1 & 3.7 & -.6 & 11 \\
\hline $1936 \ldots \ldots \ldots \ldots \ldots \ldots \ldots \ldots$ & 6.8 & 3.6 & 4.0 & 17 \\
\hline $1937 \ldots \ldots \ldots \ldots \ldots \ldots \ldots$ & 7.0 & 2.7 & 1.8 & 10 \\
\hline$\ldots \ldots \ldots \ldots$ & 1.8 & .8 & -1.0 & -11 \\
\hline $1939 \ldots \ldots \ldots \ldots \ldots \ldots \ldots$ & 6.7 & 3.6 & 4.2 & 27 \\
\hline $1940 \ldots \ldots \ldots \ldots \ldots \ldots \ldots \ldots$ & 7.8 & 3.6 & .5 & 16 \\
\hline $1941 \ldots \ldots \ldots \ldots \ldots \ldots \ldots \ldots$ & -.1 & 5.1 & 6.1 & 38 \\
\hline $1947 \ldots \ldots$ & 25.9 & 13.3 & 4.5 & 52 \\
\hline$\ldots \ldots \ldots \ldots \ldots$ & -14.8 & -6.4 & -3.5 & -53 \\
\hline $1949 \ldots \ldots \ldots \ldots \ldots \ldots \ldots \ldots$ & 3.3 & 1.2 & 1.5 & 0 \\
\hline $1950 \ldots \ldots \ldots \ldots \ldots \ldots \ldots$ & 6.8 & 4.4 & 2.8 & 54 \\
\hline
\end{tabular}

* Chicago wholesale prices.

t Chicago wholesale prices.

$\ddagger$ United States average prices.

United States average prices.

$\$$ Not available.

Sources: Butter, American cheese, and dry whole milk prices from compiled price series. Evaporated milk prices from Dairy Statistics and Related Data, U. S. Bur. Agr. Econ., Wash., D.C. June, 1951.

tion to the estimated cost values. Cheese price differences exhibit the same aspects as butter, but to a reduced degree. Since cheese undergoes an accretion in value through storage, the December price should have been for aged cheese instead of fresh. Such prices are not readily available but would normally be higher than fresh prices at a given point in time. This fact would suggest that cheese and butter storage operations were slightly lower than efficient levels during years of relatively stable demand. A certain amount of warranted pessimism could be ascribed to this type of venture. 
Price differences for dry whole milk are not very conclusive in supporting a decision on the efficiency of the storage operation for that product. This condition is probably caused by the relatively small demand involved and the magnified price effects that relatively large sporadic changes in demand produce. However, a tendency toward reasonable conformity to the storage cost estimates is evident.

Evaporated milk price differences suggest a tendency toward overstorage during the 1920's (excluding 1922). Excluding 1931, in which year sellers of evaporated milk may have been reluctant to lower prices as rapidly as butter and cheese had declined during 1930 and early 1931, the storage holdings of evaporated milk probably tended toward efficient volumes during the remaining years of the decade, and in 1940 and 1941. The negative price difference of -1 in 1934 appears to have been caused by the fixed pricing schedule of Marketing Agreement 7, which had no provision for seasonal variation. The 1938 discrepancy reflected a minor recession during the last half of that year, obviously not anticipated. Since 1947 it is evident that inaccurate expectations of demand trends led to inefficient storage volumes.

The effects of the rapidly changing conditions facing the industry are noticeable in the post-World War II period of table 13. During the war period of controlled prices, utilization distortions were caused by price incentives. After the war, readjustments took place with decontrol and an unanticipated foreign demand that continued into the first half of 1948. Late in 1948 a delayed price break occurred, again unanticipated, when foreign nations had dollar shortage problems and therefore curtailed purchases from the United States. These facts account for the large positive differences in 1947 and the large negative differences in 1948. In the spring of 1949 a government price support program was initiated for manufactured dairy products (butter and dry nonfat solids at first and cheese later). Through government purchases at specified prices, price movements were stabilized except for the rise in the established purchase price for butter in the autumn, which was accompanied by induced rises in the prices of other major products. During 1949 and over half of 1950 this support program resulted in the gradual accumulation of large stocks of dairy products. With the beginning of hostilities in Korea and the subsequent action by the United Nations, these stocks were largely eliminated by the end of 1950 . Price rises were experienced in December 1950 and continued into 1951.

The second question pertaining to the pattern of storage during seasons of increasing or decreasing prices will be investigated now. In the previous discussion about price differences some evidence suggested that expectations were not very accurate in such cases. Tables 14 and 15 give the monthly additions to storage for years of declining and increasing demand. If a declining demand is correctly anticipated by people in the industry, the resultant pattern of storage should show a delayed beginning with small volumes. Conversely, an early beginning with large volumes should occur when an increasing demand is correctly anticipated.

Generally, the butter and cheese patterns of additions to storage show no significant differences for years of declining or increasing demand. One exception might be noted for cheese in late 1941, but these additions were very 
TABLE 14

MONTHLY NET ADDITIONS TO STORAGE OF DESIGNATED PRODUCTS DURING SELECTED YEARS OF DECLINING DEMAND

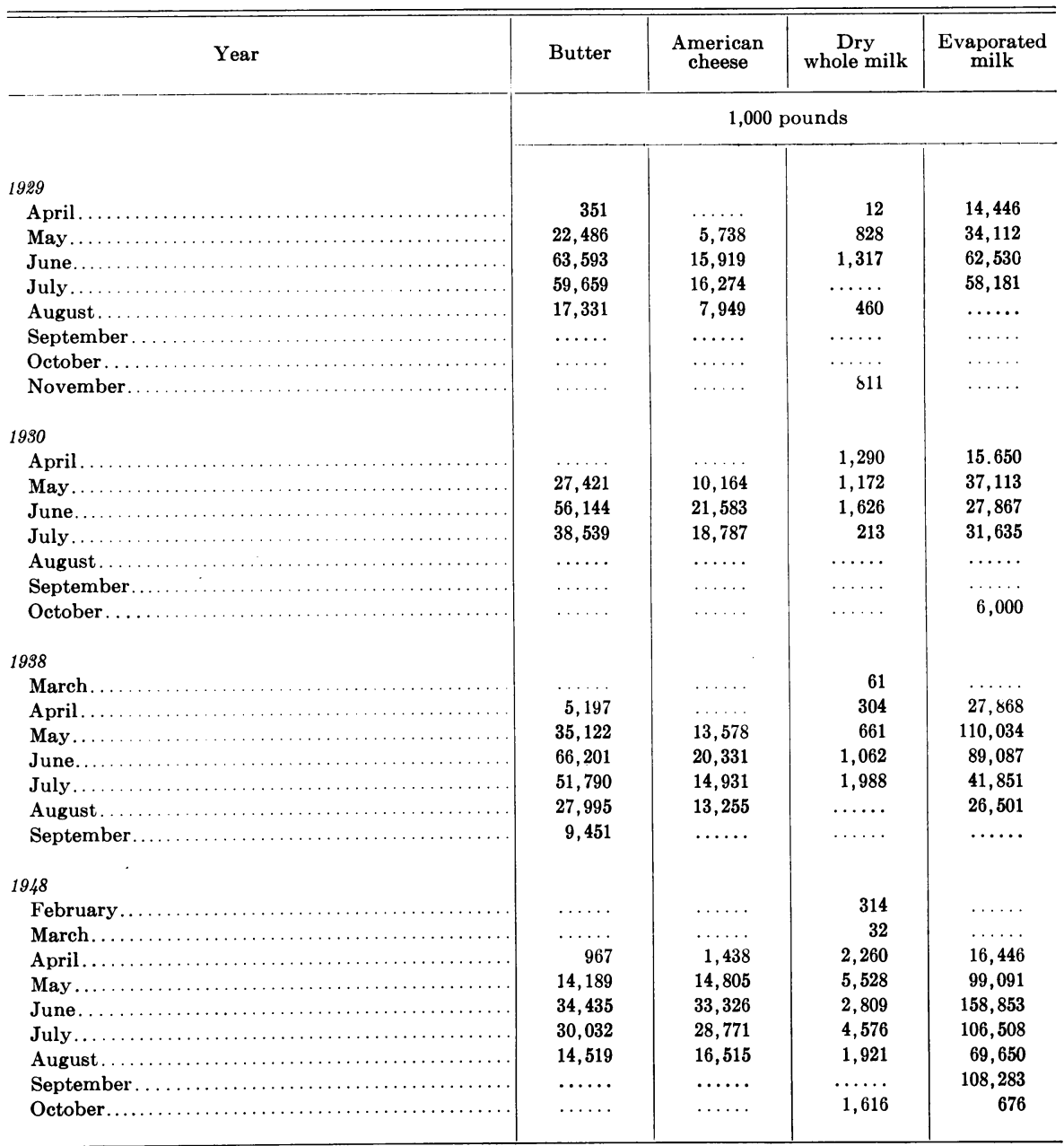

Sources: Compiled storage series.

likely undesired stocks which were accumulating with a falling demand for cheese beginning in December, 1941, and extending through most of 1942.

Patterns of additions to storage of evaporated milk and dry whole milk also fail to show efficient reactions to trends in demand. Exceptions are the patterns for 1947, which seem to reflect rational action in response to accurate expectations. The late additions for 1941 were probably due to excessive volumes earlier in the year, based on a too optimistic attitude for the rate of increasing demand. It is always difficult to determine whether additions to storage are planned or undesired accumulations. There is no doubt that the late accumulations of these products in 1948 were caused by excessive 
TABLE 15

MONTHLY NET ADDITIONS TO STORAGE OF DESIGNATED PRODUCTS DURING SELECTED YEARS OF INCREASING DEMAND

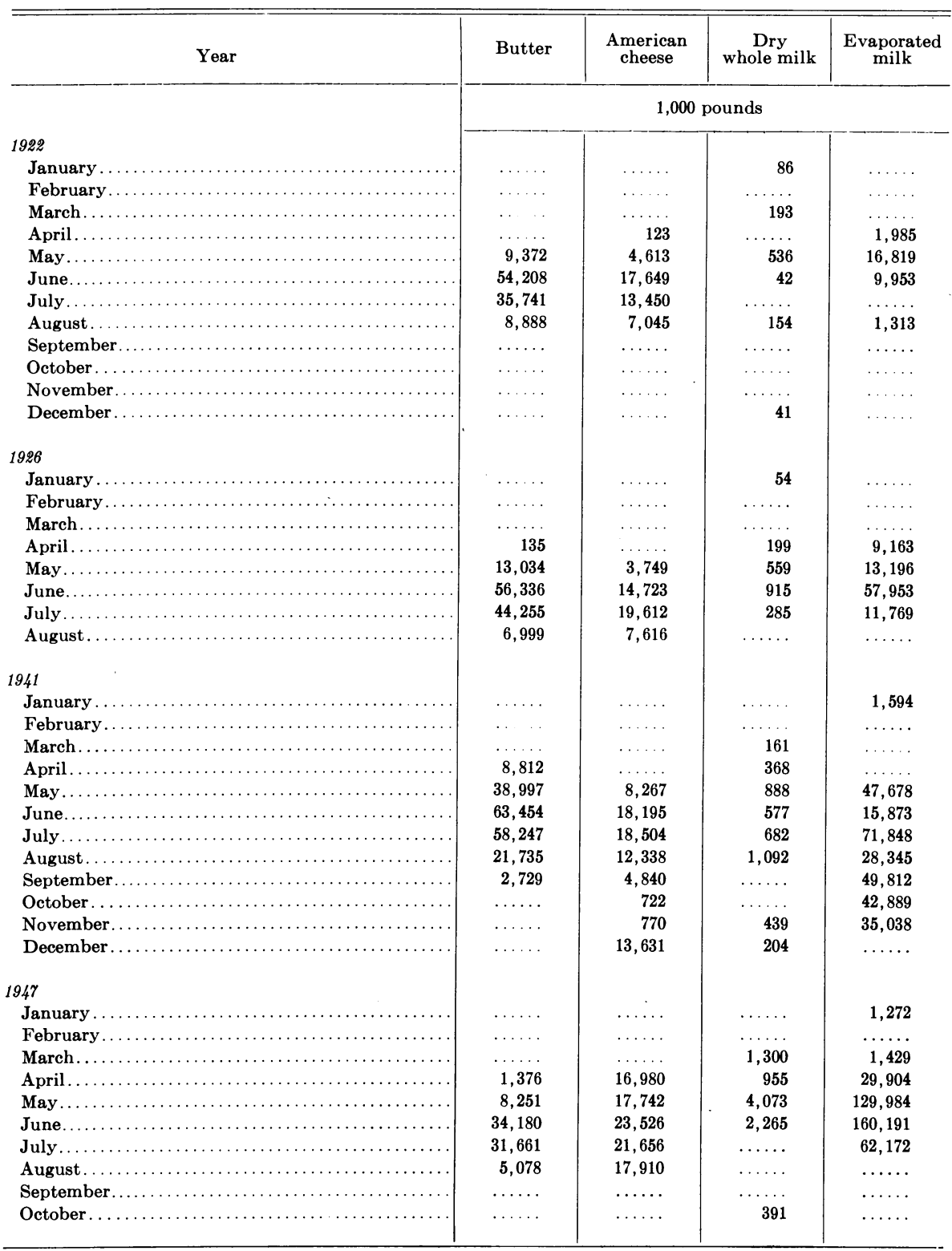

Sources: Compiled storage series. 
volumes stored earlier in the season and a continuing reluctance to adjust prices downward.

In summary, the very fact that storage has taken place and during a rational interval of the seasonal production cycle is evidence that the industry has responded to the competitive advantages of producing time utility. The discussion on price differences, estimated storage costs, and patterns of storage indicate that imperfect expectations have been followed by somewhat inefficient results. Expectations seem to be based on a stable demand structure, and this may be a very rational attitude in view of the large amount of uncertainty involved in predicting the future. However, with storage costs being relatively small in comparison with potential price variation under changing supply-demand relationships, it would appear that more efficient storage operations could have been achieved. Significant deviation from stable demands over each season is probably the reason why all products are stored and not just butter. In the case of cheese, storage over time is in part an activity directed toward a form change as well as the production of time utility.

\section{Analysis of Producer Prices for Milk and Associated Net Values in Utilization}

In section $\mathrm{V}$, the analysis of relative net prices for the major products was presented. The basic idea was to compare actual net price ratios with equilibrium net price ratios in order to determine the direction and magnitude of distortions in the relative price structure. It must be stressed again that the equilibrium ratios were determined only by means of the weight of physical yields and a few reasonable, supplementary assumptions pertaining to net prices of dry buttermilk, dry skim milk, and dry whey relative to the net price of butter. Since the weights and supplementary assumptions were nearly unrelated to the price level, so were the equilibrium ratios. The equilibrium condition, upon which the solution for ratios was based, was the equality of net values per unit of milk in all types of plants. A simultaneous equilibrium condition was also stated previously but not required for the analysis of section $\mathrm{V}$, namely, the common net value would be equal to a single price paid for milk f.o.b. plant locations. Of course, with varying average fat content for milk at different plants, the last condition had to be extended to a price schedule that was a function of the fat test. It was only in the case of cheese plants that complications arose in securing net price ratios that were not variable with the fat test of the milk.

Potential paying ability versus actual payments. Section V, therefore, was concerned with relative net prices which would equate the "potential" paying ability of the various types of plants for milk of comparable composition. Additional elements of pricing efficiency must be investigated. In an actual situation of equilibrium net prices for the final products, there is the possibility that prices paid for milk will diverge from the common, potential paying ability of the plants. Under such conditions two important aspects of the pricing structure should be analyzed for competitive efficiency: (1) do all the types of plants actually pay on a single price schedule? and (2) (with 
or without an affirmative answer to the first question) how closely does the actual price paid to a producer come to the realized net value estimated for a given type of plant? This section will be devoted to developing answers to these two questions.

With disequilibrium relative net prices for products having been the normal situation, as was shown in section $\mathrm{V}$, interesting implications arise in reference to the two questions above. Excess productive capacity for all types of plants in the industry has been and probably will always be a prevalent characteristic. When relative prices are distorted, the level of the paying schedules by types of plants should be positively correlated with the ranked advantages of such distortion. Plants with output which is relatively high in price should tend to outbid other types of plants in response to the profit motive. Operating at undercapacity volumes, such plants could gain from an increase in receipts of milk, even with a rising price, provided the rise in their paying price for the raw milk was more than offset by processing cost reduction with increasing volume, and the price did not exceed the net value of the milk in that use.

Definition of paying schedule. Perhaps a fuller explanation of a paying schedule, as used here, would be useful. In this paper the term means the schedule of prices for 100 pounds of milk as the latter varies in fat content. Further, such schedules will be assumed (in conformity with actual practice) to be linear functions of the fat test. Graphically, these schedules may be visualized with the price per 100 pounds of milk $(P)$ as the ordinate and the fat test of milk $(F)$ as the abscissa. An interesting paradox arises with linear paying schedules. Consider a plant that has a weighted average fat test for milk receipts during a given month equal to 4 per cent. Using accurate cost estimates and relevant selling prices for final products, the plant manager estimates the aggregated milk had a net value of $\$ 4.00$ per hundredweight and wishes to return the total net value of the milk to his producers. Any linear paying schedule through a price of $\$ 4.00$ and a fat test of 4 per cent would accomplish this objective. This is true, since the weighted average price per hundredweight of milk for a lot of milk made up of various quantities of differing fat content would be $\overline{\boldsymbol{P}}_{\mathrm{w}}=a+b \overline{\boldsymbol{F}}_{\mathrm{w}}$ (where $\overline{\boldsymbol{F}}_{\mathrm{w}}$ is the weighted average fat test) if milk of each fat test were paid for by a schedule of the form $P=a+b F$. It is obvious that an infinite number of consistent values of $a$ and $b$ can be found to satisfy $\overline{\boldsymbol{P}}_{\mathrm{w}}=a+b \overline{\boldsymbol{F}}_{\mathrm{w}}$ when $\overline{\boldsymbol{P}}_{\mathrm{w}}$ and $\overline{\boldsymbol{F}}_{\mathrm{w}}$ are fixed. However, only one schedule (as an average relationship) would be equitable to the individual producers, and this schedule would be identical to the net value schedule for milk of varying composition for that plant.

Before World War II, the usual type of paying schedule at manufactured dairy product plants using whole milk was a direct ratio to fat schedule. Such a schedule would be of the form $P=b F$, where $P$ is the price per 100 pounds of milk with fat content of $F$ pounds and $b$ is the price per pound of fat. Some cheese plants and butter skim milk plants did employ paying schedules of the form $P=a+b F$. Plants using the direct ratio schedule attempted to

${ }^{4}$ The Midwest code formula at condenseries for $F>4$ was also of this type. For $F<4$, a code formula of the direct ratio type was specified. Since actual paying prices were generally higher than the code prices, neither code formula was effective except for a few months. 
set the value of $b$ to be equitable for a fat test comparable with the average of their aggregated receipts, but were overpricing milk of greater fat content and underpricing milk of lesser fat content. With high and low fat milk being highly correlated with breeds of cattle, the effect of such distorted pricing would tend to distort the short-run relative prices of cows by breeds and the long-run numbers by breeds. If farmers were fully aware of price advantages at alternative plants, it would appear that the competitive advantages to a plant of utilizing a paying schedule of the form $P=a+b F$ would eventually overpower plants of the same type which attempted to price with a direct ratio schedule in the same area, provided $a$ and $b$ have values consistent with the net value of milk of various fat tests.

Although the subject discussed in the last paragraph could be expanded into a major analysis alone, its inclusion here is primarily for definitional purposes. An attempt will be made now to determine the coincidence or lack of coincidence of the paying schedules for milk at plants of various types in the Midwest area during the past. Data, such as they are, are available only for butter, American cheese, and condensing plants. Therefore, dry whole milk plants will not be covered.

Limitations of available data. To secure a good answer to the first question, actual paying schedules would be required in an area where plants of the various types were located. This type of information is not generally available so one must resort to a less accurate approach. For the years 1934, 1936, and 1938-1942, inclusive, state-wide average prices per hundredweight of milk and associated fat test at Wisconsin butter, cheese, and condensing plants are available (Gilbert, 1945). Assuming "direct ratio to fat" paying schedules, cheese plants and condenseries paid, respectively, about 2 and 4 cents per pound fat above the price paid at butter plants during most of this period. Also, the average fat test of milk received at these plants was consistently ordered from low to high as: (1) cheese plants ; (2) condenseries ; and (3) butter plants. Under such circumstances, a single schedule of the form $P=a+b F$ would necessarily mean that the price paid per pound fat would be highest at cheese plants, next highest at condenseries, and lowest at butter plants (both $a$ and $b$ assumed positive). If the butter and cheese plant paying prices fell on this schedule, the condensery price still would be higher by a small amount. From a between-type of plant point of view, it would appear that a paying schedule of the latter form is more relevant, and that deviations from a single schedule of average prices per hundredweight of milk of average fat content have not been great.

Beginning in 1947, a more complete set of producer prices and associated average fat tests has been gathered by governmental agencies. ${ }^{5}$ These data are state-wide averages, covering an extended number of states, and are given for butter, American cheese, and evaporated milk operations. The data for the last operation start in August, 1947. On a direct ratio basis, evaporated milk and American cheese plants paid about 3 cents per pound fat more for milk than did butter plants during late 1947 in the Midwest area. During

\footnotetext{
${ }^{5}$ Data on butter and cheese plants secured from: U. S. Bur. Agr. Econ., Milk prices paid at creameries and cheese factories. Wash., D. C. Data on evaporated milk plants secured from: U. S. Bur. Agr. Econ., Evaporated, Condensed, and Dry Milk Report. Wash., D.C.
} 
1948, condenseries and cheese plants paid on the average, respectively, about 7 and 3 cents per pound fat higher than butter plants. Similar figures for 1949 and 1950 and in the same order are (2 and -2$)$ and $(4$ and -1$)$ cents per pound fat. With a between-plant paying schedule of the form $P=a+b F$, it would appear that butter and evaporated milk plants were nearly paying on a basis equivalent to a similar schedule during 1947-1950, but that American cheese plants were paying slightly lower prices. It is probably true that most plants were using an internal paying schedule of the above form during the postwar period.

Preliminary conclusions on producer prices. The preceding information supports a conclusion that prices paid for milk at various types of plants have diverged. Generally, evaporated milk plants have been paying higher prices for their raw milk than have other manufacturing plants. This leads to the second question pertaining to the accord between prices paid for milk and estimated net values realized in utilization. Section V concluded that evaporated milk prices have been too high relative to butter and cheese if realized net values for milk were to be equated in all uses. Recognizing that evaporated milk plants have paid more for their milk, the problem of whether this higher price for milk was compatible with the high relative price of evaporated milk remains to be investigated.

Net margins (positive or negative). By means of computations estimating differences between the realized net values and the prices paid for the raw milk, figures 15 through 21 have been drawn for summary purposes. Net value estimates were made by direct substitution into the formulas given in section IV. These computations were based on state-wide averages, both for milk prices and fat tests, and do not reflect movements along any paying schedule for a given type of plant. Consequently, the calculations only show whether the plants were setting their average price over or under their average realized net value for aggregate receipts. The evaporated milk comparisons are primarily representative of national brand operations.

From figure 15, it appears that evaporated milk plants were realizing about 40 cents per 100 pounds of milk in net value over prices paid producers during all the prewar years that were considered. In spite of the relatively high producer prices paid, evaporated milk plants still retained the highest margins of realized net value over raw milk cost.

According to figure 16, cheese plants were apparently paying about 15 cents per hundredweight more for their milk than they were realizing in net value. Adjustments of the basic calculations, in accord with reasons given in section IV, for externally supplied boxes and increased moisture (37 to 39 per cent) allowances in cheese would raise the lines of this figure by about 10 cents per hundredweight of milk. This would reduce the average loss to about 5 instead of 15 cents. Erratic positive margins are exhibited for Wisconsin butter plants in figure 17. Prior to 1938, the valuations placed on skim milk in the calculations were probably excessive for state-wide average calculations. On this basis a reduction of 10 to 15 cents per hundredweight in the level of the lines for 1934 and 1936 might be more reasonable. With such an adjustment, the prices paid for milk at butter plants appear to have been approximately equal to realized net values except in 1942. During 1942, an average 

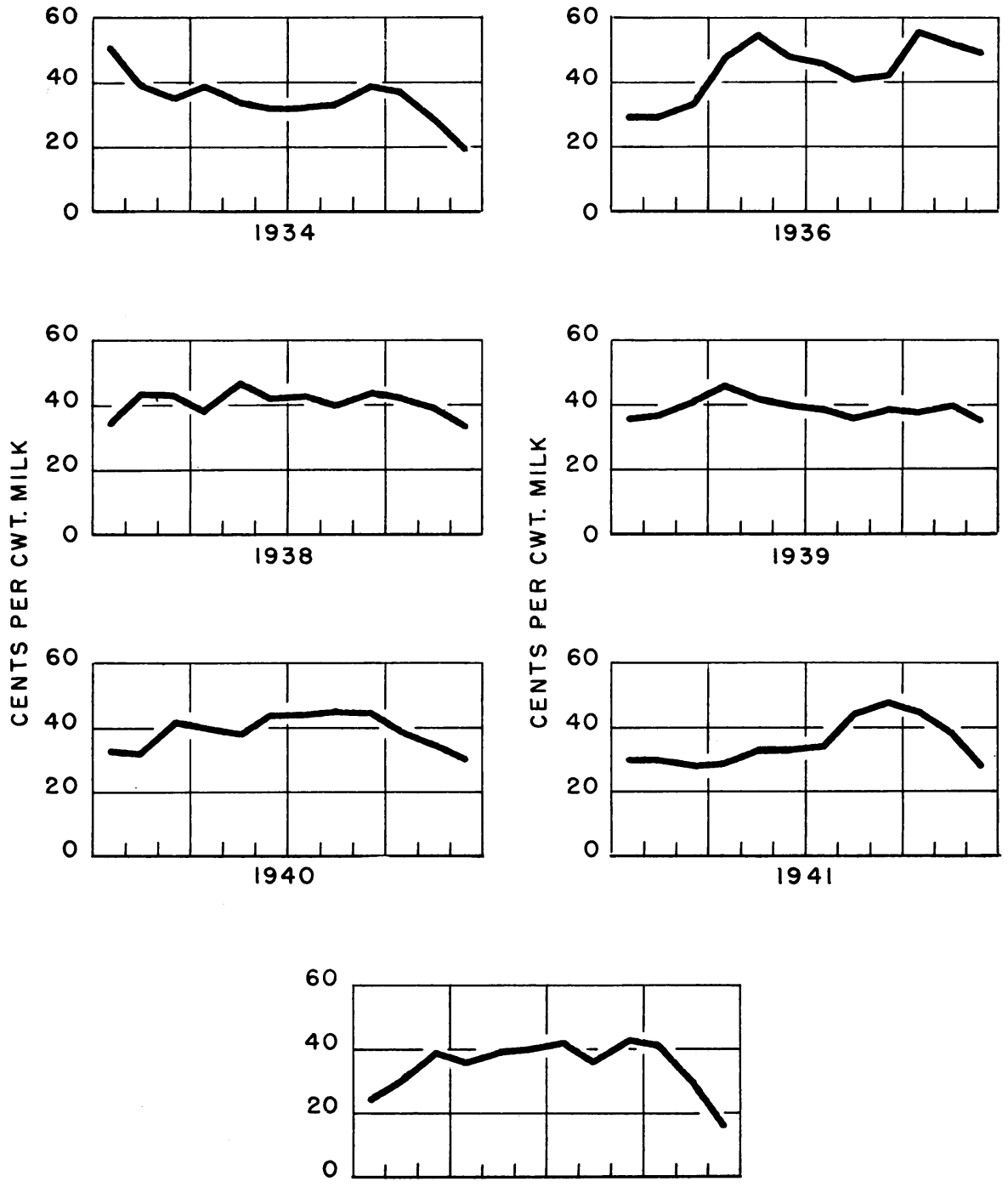

1942

Fig. 15. Margins of estimated net value of products over price paid per hundredweight milk at Wisconsin evaporated milk plants, by months. 

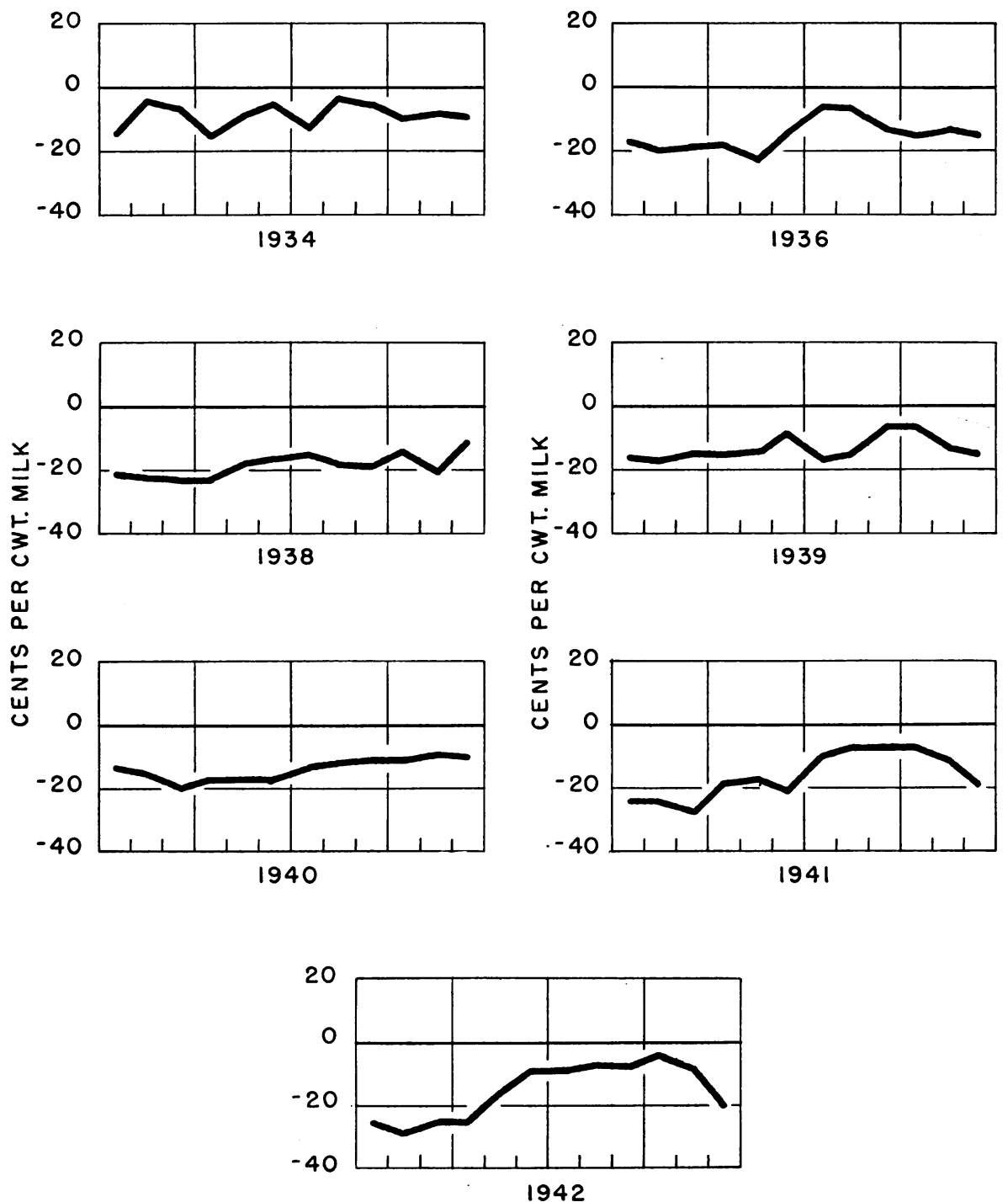

Fig. 16. Margins of estimated net value of products over price paid per hundredweight milk at Wisconsin cheese plants, by months. 

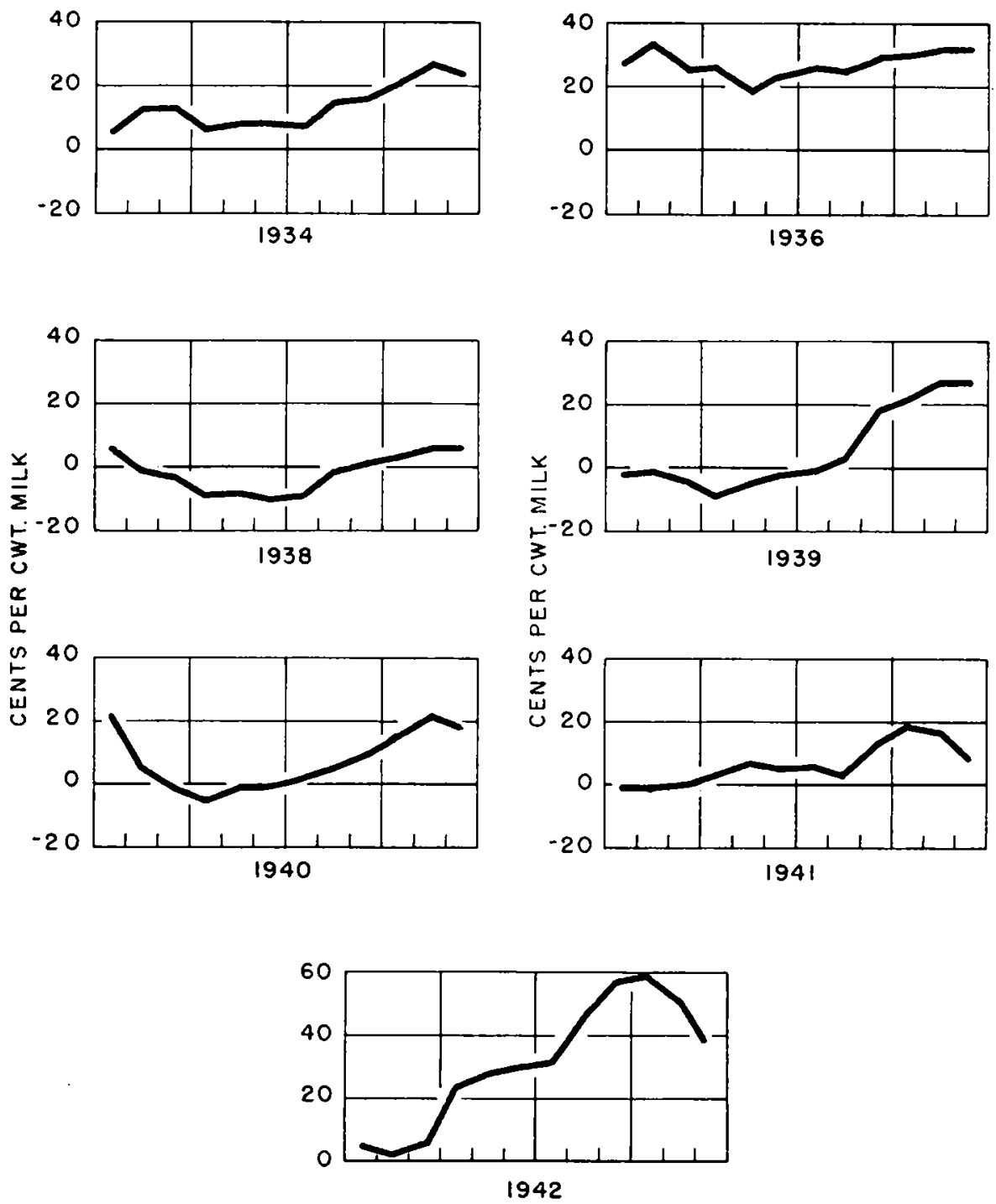

Fig. 17. Margins of estimated net value of products over price paid per hundredweight milk at Wisconsin butter plants, by months. 

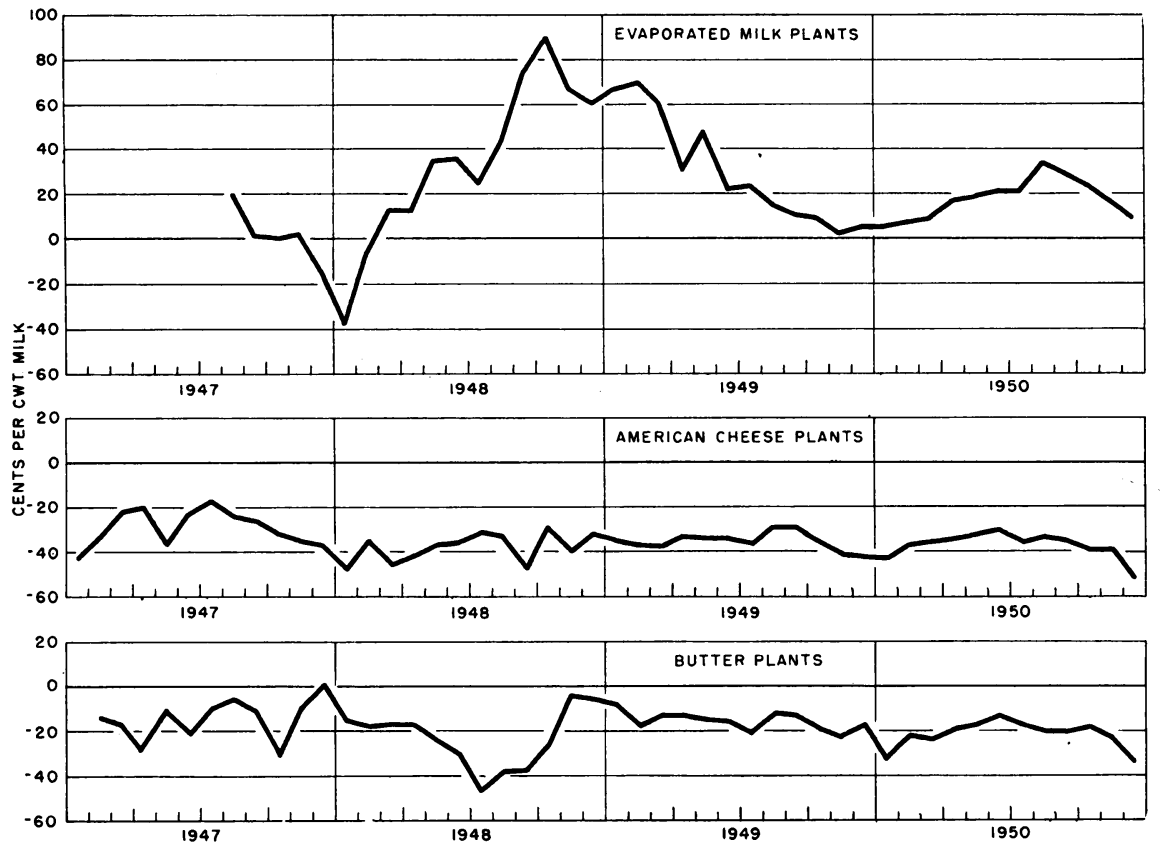

Fig. 18. Margins of estimated net value of products over price paid per cwt milk at Wisconsin plants, by months.
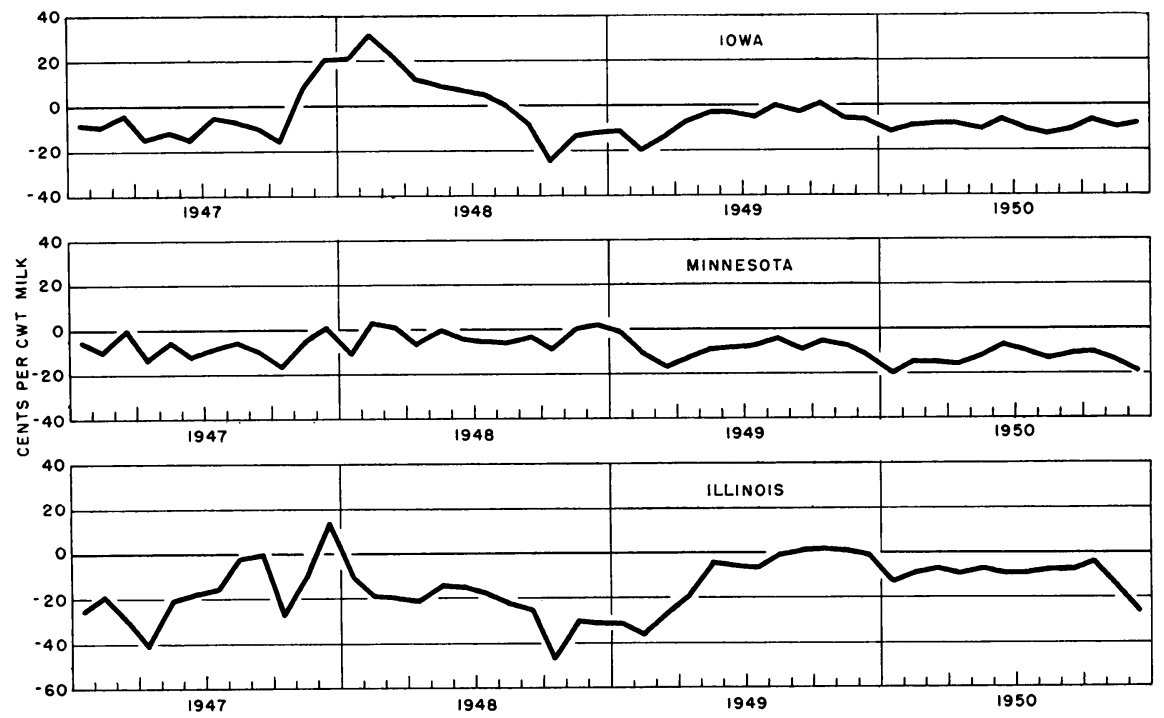

Fig. 19. Margins of estimated net value of products over price paid per hundredweight milk at Midwest butter plants, by months. 


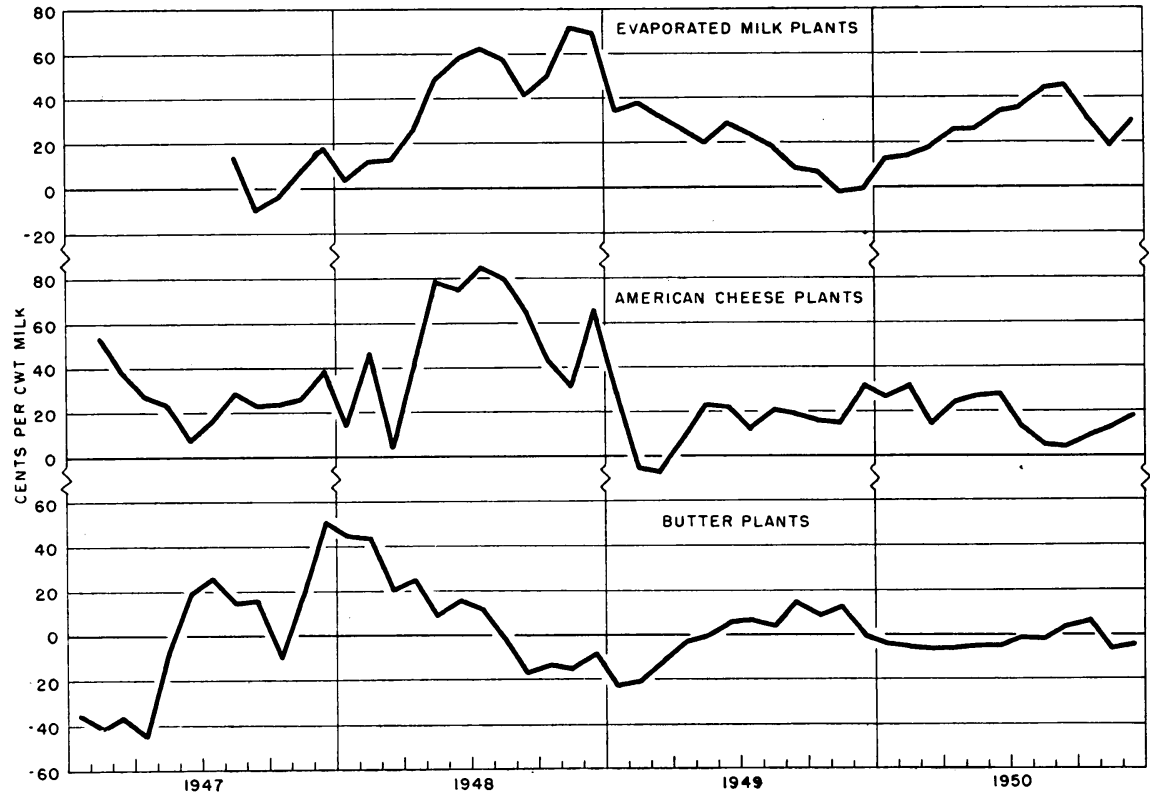

Fig. 20. Margins of estimated net value of products over price paid per hundredweight milk at California plants, by months.
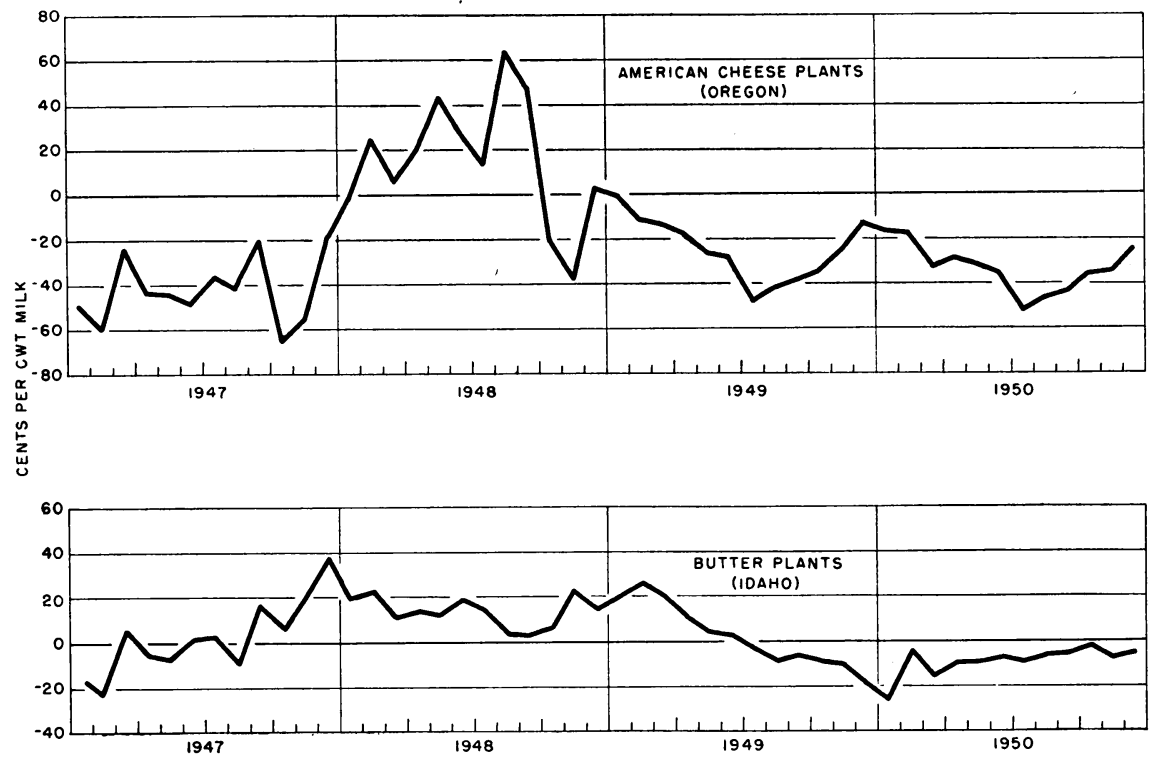

Fig. 21. Margins of estimated net value of products over price paid per hundredweight milk at designated plants, by months. 
value of about 25 to 30 cents per hundredweight was retained by the plants.

Are these same conclusions valid for the postwar years 1947 through 1950 ? Figures 18 and 19 give basic information for answering this question with respect to the Midwest area. The upward adjustment of the cheese line would amount to about 25 cents during these years of high prices. Except for a few months late in 1947 and early in 1948, positive margins were experienced at evaporated milk plants. Wisconsin cheese plants (after the above adjustment) had losses of about 10 cents per hundredweight of milk while butter plants in that state were undergoing losses averaging nearly 20 cents per hundredweight of milk used.

Butter plants in Iowa, Minnesota, and Illinois were also sustaining losses during this period, as shown in figure 19. The level of such losses in Minnesota and Iowa was smaller than in Wisconsin and Illinois, although the latter state adjusted quite efficiently after the initiation of the butter price support program in 1949. It is likely that these losses are underestimated in all instances for butter plants because of the use of a high skim milk valuation that may not be pertinent to a state-wide average analysis.

Further investigations of the relationships between realized net values and producer prices were made from data for states in the Western region. The results of such analyses are given in figures 20 and 21. In California, the situation for evaporated milk was quite similar to that for the Midwest. Small volume and isolated location are believed largely responsible for the high positive margins at California cheese plants. An environment suitable for monopsonistic purchasing of milk probably existed for plants producing this product. California butter plants have been relatively efficient in returning to producers the net value of milk which they used. During the rapidly rising price period of 1947 and early 1948, however, the adjusting process seemed to be erratic, but stabilized with less violent changes in margins thereafter.

In Oregon, from one third to one half of the total American cheese production has been manufactured by one coöperative association-the Tillamook County Creamery Association. Cheese from this organization tends to have a slight premium price over other local production and over imports from the Midwest. These premiums were about 4, 2, 3, and 4 cents per pound in the years 1947, 1948, 1949, and 1950, respectively. The calculations for cheese plants in Oregon, shown by the upper part of figure 21, estimated f.o.b. plant prices by means of the wholesale cheese prices in San Francisco, from which were subtracted freight and handling costs. This may have been valid for some plants other than those of the Tillamook Association. It would appear that plants producing nonpremium cheese have been suffering losses. Tillamook plants with premium sales would have about $40,20,30$, and 40 cents added to the margins line of figure 21 during the four years 1947 to 1950 . With this adjustment, such plants would have been retaining from 20 to 80 cents per hundredweight of milk during the first nine months of 1948 but would have been oscillating around a zero margin during the other portions of the postwar period.

Idaho butter plants seem to have been rather efficient in paying producers the net value of their milk. A slight amount of sluggishness is evident from the long periods of plus or minus margins before a crossing of the zero line 
occurred. However, considering the small magnitude of all deviations from zero, this fault has not been serious. A slight improvement of the results might have been achieved by using Los Angeles instead of San Francisco as the basing point for butter prices. Although Idaho butter is shipped to both of these markets, Los Angeles is the greater outlet.

Final conclusions on producer prices. Evidence for both the Midwest and western areas shows that the higher price paid producers for milk at evaporated milk plants was not sufficiently large to be in accord with the high relative price for the final product. Again, these conclusions are primarily valid for the national brand operations. With a differentially lower product price, local brand operations probably came quite close to paying out the competitive net value of milk which they used.

Producer prices and the Evaporated Milk Code. Under Marketing Agreements 7 and 60, minimum producer price formulas were established for the evaporated milk industry. It seems appropriate to investigate the competitive relevance of these formulas. Two sets will be considered, namely, those in effect for the Midwest and for California areas under Agreement 60 and License 100. By simplifying the original formulas, they can be written as:

1. Midwest fờrmulas

(a) For milk used for evaporated milk, and $F<4$,

$P=\left(1.1143 p_{\text {b, Chicago }}+.4457 p_{\mathrm{c}}\right.$, Plymouth $) F$

(b) For milk used for evaporated milk, and $F>4$, $P=\left(.9429 p_{\text {b, Chicago }}+.3771 p_{\mathrm{c}}\right.$, Plymouth $) F+$

$\left(.6857 p_{\mathrm{b}}\right.$, Ch icago $+.2743 p_{\mathrm{c}}$, P lymouth $)$

(c) For milk used for other products,

$P=\left(.9429 p_{\text {b, Chicago }}+.3771 p_{\mathrm{c}}\right.$, Plymouth $) F$, or current price paid at plants of similar use-whichever is greater.

2. California formulas

(a) For milk used for evaporated milk, $P=\left(1.20 p_{\text {b, San Francisco })} F\right.$

(b) For milk used for other products, $P=\left(1.10 p_{\text {b, San Francisco }}\right) F$, or "current price paid at plants of similar usewhichever is greater.

In all instances, a weighted average blend price would be used if multiutilization occurred. Only one of the above formulas was of the form $P=$ $a+b F$, the others being of the direct ratio to fat type.

Considering the Midwest formulas first, it should be noted that $(b)$ and (c) have identical multipliers of $F$, but that the last term of $(b)$ places formula $(b)$ prices above formula $(c)$ results by a positive amount. For the years 1936 through 1942, the annual average differences were about 26, 27, $22,21,24,28$, and 33 cents per hundredweight of milk. During these years, the Plymouth cheese price consistently averaged about one half the Chicago butter price. Injecting this relationship into the Midwest formulas results in:

(a) $P=\left(1.3372 p_{\text {b, Chicago }}\right) F$

(b) $P=\left(1.1315 p_{\mathrm{b}}\right.$, Chicago $) F+\left(.8228 p_{\mathrm{b}}\right.$, Chicago $)$

(c) $P=\left(1.1315 p_{\mathrm{b}}\right.$, Chicago $) F$ 
From the net value formulas developed in this paper, the equation for the butter operation under conditions of a zero net value for skim milk would be approximately $P=\left(1.2267 M_{\mathrm{b}}\right) F$. With $M_{\mathrm{b}}$ equal to $p_{\mathrm{b}}$, Chicago minus about 3 cents transportation and processing cost per pound of butter, this equation and $(c)$ above are quite compatible. In other words, equation (c) for the Midwest area was approximately equivalent to the formula for net value of fat used in a butter operation. Further, the positive additions in $(b)$, as given in the last paragraph, were consistent with the skim milk values estimated for these years. This means that $(b)$ was a consistent pricing formula for a butter-dry skim milk operation but, since the price of evaporated milk was relatively high, the latter operation did not find it difficult to pay slightly above this schedule.

Pricing formula $(a)$ was equal to 1.18 times $(c)$. Assuming that any plant would be indifferent to the internal pricing schedule for its patrons (providing such schedule did not have a price at the aggregated average fat test for the plant which was above the average net value of the receipts), a direct ratio formula like $(a)$ would be acceptable. Pricing formula $(a)$ gave identical results with $(b)$ at $F$ equal to 4 . If $(b)$ were extended as a net value function for $F<4$, it would lie above the price schedule given by $(a)$. Now, the reported average fat test at Midwest plants (evaporated and butter) was below 4 per cent (about 3.8) so that a butter-dry skim milk plant, even using a direct ratio price schedule, could alternatively pay slightly more than was indicated by formula $(a)$. Formula $(b)$ was ineffective since the average fat test at plants never became high enough. This formula would have been reasonable had it applied to the lower fat test range. The conclusion to be drawn is that none of the Midwest pricing formulas produced results high enough to have been effective indicators of the advantages of diverting milk to evaporated milk plants. An evaluation based on utilization by cheese plants would have led to the same conclusion.

The pricing formulas for California condenseries were both of the direct ratio to fat type, with the first being 1.09 times the latter. Through calculations similar to those used in evaluating $(c)$ for the Midwest, it can be shown that the second formula for California was consistent with the net value realized from fat in butter plants. However, it does not include some positive value for the skim component of the whole milk. The difference between the prices calculated by the first and second formulas at $F$ equal 5 would be about $0.50 p_{\mathrm{b}}$, San Francisco. Even at this high value for the fat test (much above the average fat test at California plants), the value of the skim milk would be greater than one half the price of a pound of butter, so that the first formula underestimated the additional value of the skim component at a butter plant. The same conclusion is reached, then, for the California pricing formulas as was reached for the Midwest. Prices established by these equations were too low in the sense of opportunity cost schedules. With the high relative price of evaporated milk, condenseries could easily exceed these schedules. It is interesting to note that none of the pricing formulas used prices for evaporated milk as value indicators for milk, even though this would have seemed a direct approach to the problem. Basing the pricing formula for milk used in the production of evaporated milk on the product would seem reasonable. 


\section{Some Locational Aspects of the Relative Price Structure for Manufactured Dairy Products}

The relative price analysis given in section $\mathrm{V}$ held for the particular geographic area that was outlined in the Midwest. Arguments were presented at that point to support the reasonableness of such a location as an approximation to a multiple-use area of relatively small dimension. The economic feasibility of multiple use was the basic criterion in conjunction, of course, with smallness of the geographic size of the selected area. Should any other area in the United States satisfy these conditions, the equilibrium ratios of the net prices of the major products as developed in section $\mathrm{V}$ would be applicable. Specific estimates of the actual ratios would have to be calculated before pricing efficiency comparisons could be made. If standardization of milk were permitted in the cheese operation, then that condition would have to be imposed in the computation of the equilibrium ratios, since this would affect the yields of the products.

It should be pointed out that the relative price analysis for the Midwest was based on the condition of equal economic advantage for the utilization of milk in all four of the major types of processing plants. It is highly unlikely that any other area in this country would approximate an economically feasible four-use area. However, there are numerous areas where equal advantage would hold for two or three uses. The analysis of relative prices for such areas would proceed as in section $\mathrm{V}$, but only the reduced number of operations would be included.

Geographic variation in relative net prices. Although the geographic structure of product prices (section IV) was analyzed separately from the relative price structure (section $\mathrm{V}$ ), the two pricing aspects are definitely interdependent. Except for incomplete coverage of evaporated milk and dry whole milk, the geographic structure of product prices appeared to be consistent with competitive results. Neglecting geographic variations in processing costs, the zonal nature of evaporated milk prices in conjunction with competitive geographic variation in cheese and butter prices tends to reduce the distorted high relative price of evaporated milk with respect to butter and cheese as one considers supply areas of the United States other than that of the dominant Midwest area. Evidence will now be presented showing that the nearly flat geographic structure of evaporated milk prices does tend to reduce its comparative advantage in utilization of raw milk over butter, for example, at supply areas located at great distances from the Midwest. Other evidence will be given, which suggests that the reduction in comparative advantage fails to remove the absolute advantage of the evaporated milk operation. These remarks are directed toward the national brand segment of the evaporated milk industry.

The supply area selected for comparative purposes with the Midwest is the San Joaquin Valley of California. Since the early 1930's, this area (and other states of the Western Region) has not been supplying the total butter requirements of coastal markets and has been in local competition with Midwest producers of this product. The locational advantage of California butter plants has been greater than the disadvantage of higher processing costs so 
that the net price of butter has been slightly higher at California supply points than at Midwest plants. With respect to evaporated milk pricing zones, California was in Zone II and the Midwest area in Zone I. Historically since 1932 (except in 1936, 1937, and 1938), evaporated milk prices have been slightly higher in California than in the Midwest, but higher processing costs have caused net prices to be reversed in order of magnitude.

The average fat test of milk used in California has been about 0.2 per cent higher than in the Midwest. However, for a fixed value for $r_{\mathrm{n}}$ (the ratio of the net price of dry skim milk to the net price of butter) the equilibrium ratio of the net price of evaporated milk to the net price of butter does not vary with the fat test (see the evaporated milk line on figure 12). The historical estimates of $r_{\mathrm{n}}$ for California and for the Midwest have been essentially equal at each point in time. Consequently, the equilibrium ratios for evaporated milk relative to butter have been approximately equal in the two areas, and the simple ratio of (1) the estimate of the actual ratio of the net price of evaporated milk to the net price of butter in California to (2) the same ratio in the Midwest can be used as a measure of comparative advantage. If (1) divided by (2) is less than unity, then the indication is that the comparative advantage of producing evaporated milk instead of butter is smaller in California than in the Midwest.

Geographic variations in relative net prices indicate comparative advantage. The computations suggested above were made, and since 1932, the ratio of (1) to (2) has varied between 0.8 and 1.0. The lower values dominated the period prior to World War II and the higher values occurred since that time. In any event, the general evaluation would place the comparative advantage of the evaporated milk operation over the butter operation in the Midwest instead of California. It should be pointed out that if some other supply area of Zone I (with a butter deficit condition) were employed instead of California, then the evidence on the same ranking of comparative advantage would be even stronger.

The above discussion on comparative advantage only involved a comparison of the relative price structure between two geographic areas. Given the distortion pointed out in section $\mathrm{V}$ for the Midwest relative prices, the indications are that the same distortion has been less in California. The final issue is to assess how much the distortion in the relative price structure has been reduced at California points of supply and whether it has reached equilibrium levels. The answer to this problem is equivalent to ascertaining whether the evaporated milk operation has had an absolute advantage over other operations in the utilization of manufacturing grade milk in California.

In short, the last issue was answered by a relative price analysis similar to the development in section V, but for the California area. All the particular aspects (fat test of milk, processing costs, and standardized cheese production) related to the latter area were taken into consideration. The quantitative details of the comparative results need not be presented since they were very similar to the Midwest solution. The relative price structure had approximately the same elements of distortion but in a slightly reduced degree. Evaporated milk net prices were definitely high relative to butter and cheese net prices and indicated strong absolute advantage in the utilization of manu- 
facturing grade milk in the production of the first product at California supply points.

Advantage of evaporated milk operation. The evidence presented in the previous paragraph suggests that the geographic aspects of the price structure for the manufactured dairy products that tend to favor the production of the low valued products at supply points away from the Midwest area probably never resulted in changes in the relative price structure of sufficient magnitude to eliminate the absolute advantage of the national brand evaporated milk operation. This judgment does not apply to geographic locations where the production of evaporated milk is not economically feasible because of the location of other supply areas for the product in relation to the consumption points.

\section{Conclusions}

As the analyses in the preceding sections were developed, numerous specific judgments were made on the basis of comparative evidence. Instead of repeating these specific conclusions for the manufactured dairy products industry, a few general comments will be followed by a discussion of the possibilities of applying the procedural techniques which have been outlined for other economic investigations. Finally, an opinion will be expressed as to the directions which future methodological developments should take so that analytic techniques will conform more closely with reality.

Although some persistent inconsistencies in the price relationships of the manufactured dairy products industry were disclosed, much of the evidence suggested that the pricing mechanism was remarkably compatible with a competitive system. The national brand evaporated milk segment of the industry appeared to be unique in its general divergence from price results expected in a competitive norm. It is believed that the ice cream processing segment would have been similar in this respect. Lack of homogeneity in this activity and of suitable price data, however, made the inclusion of this sector of the industry impossible.

There is no need in the present study to propose any remedial programs for removing the inconsistencies in the price structure of the industry. The initial intent of this study was to develop techniques and make quantitative measurements of industry pricing and not to venture into the realm of policy. Quantifying what may have been qualitatively sensed could have a strong influence by itself on policy and action programs. Moreover, the results of this study do suggest areas where future studies may profitably investigate the institutional framework of the industry in order to contribute to such policy recommendations.

What are the possibilities of using the procedures outlined in this study for other industry price analyses? Many other agriculture products are produced under conditions functionally similar to those of the manufactured dairy products industry. Wherever a situation is found in which a homogeneous raw material is actually being used in the production of alternative, standardized, final products, the procedures used in this study are applicable. It is possible that greater difficulties may exist for joint cost allocations, of course, and that numbers and interdependencies of firms may provide a set- 
ting in which actual price results are likely to deviate from the efficiency values. Still, this does not reduce the applicability of a pricing model based on a competitive system. Such a comparative pricing model is useful in describing what could happen and is not posed to describe what will happen. Should the actual pricing results differ from those expected by the model relationships, then this discord merely points out possible areas for policy decisions and action. The latter decisions may or may not be directed toward changing the situation.

Aside from fields of agricultural processing operations, certain forest utilization operations, mineral operations, and petroleum operations might fall into the category of applicability. A careful investigation of the productive activities would have to be made in each case in order to substantiate the use of the model. More generally, the model has application in broader areas. Since it is simply an aspect of interdependent pricing, it could be expanded to cover the general equilibrium system. However, as soon as heterogeneity of inputs occurs and the field cuts across industries, then the complexities make practical applications increasingly difficult.

An allusion to the appropriateness of static methodology was made near the end of section III. Static techniques are logically inconsistent with the real economic system of dynamic interaction. The time dimension is vital in a system where instantaneous adjustments are impossible. Consequently, future developments in economic methodology must establish theoretical models on a basis in which time is an explicit variable. By means of deductive reasoning from the time paths of interdependent economic values, the implications of velocities, accelerations, et cetera, may be established. Such procedures will require a change in data collection from present arbitrary, calendar period methods. In other words, relevant data will be required in order to proceed in an empirical, deductive way. For velocity data, the minimum period of time required to specify a rate of operation would be the calendar period essential for such values. This calendar period would vary from one type of productive activity to another. Since one would probably estimate accelerations from finite first differences in velocity values, it is obvious that velocity data must be temporally valid and not an aggregation over some arbitrary time interval.

A careful inclusion of the effects which biological facts, social customs, and political institutions have on the duration intervals of active production and consumption must be made. Such considerations are essential since economic activity is interdependent with these noneconomic aspects.

It is hoped that future developments will assist the economist in supplying the social group with information that will lead to more efficient decisions. 


\section{REFERENCES CITED AND RELATED LITERATURE}

ARRow, K. J.

1951. Social choice and individual values. Cowles Commission Monog. 12:1-99. John Wiley and Sons, Inc., New York City.

BAKer, B. A., and R. K. Froker.

1945. The evaporated milk industry under federal marketing agreements. Wisconsin Agr. Exp. Sta. Res. Bul. 156:1-91.

BLACK, J. D., and E. S. GUTHRIE.

1924. Economic aspects of creamery organization. Minnesota Agr. Exp. Sta. Tech. Bul. $26: 1-111$.

BREMS, HaNs.

1952. A restatement of the Jantzenian laws of production and cost. 21 p. Univ. California, Dept. Econ., Berkeley, Calif. (Mimeo.)

BRESSLER, R. G., JR.

1950. Efficiency in the production of marketing services. Univ. Chicago Econ. Efficiency Ser. Monog. 7:1-81.

BRUBAKER, D. D.

1943. Cheese yields-a summary of available information. U. S. Farm Credit Admin. Spec. Rpt. 124:1-13. Wash., D.C.

Caparoon, C. D.

1951. Wisconsin farm milk prices by markets. Wisconsin Dept. Agr. (in coöperation with U. S. Dept. Agr.) Spec. Bul. 6:1-36.

CASSELS, J. M.

1937. Study of fluid milk prices. 303 p. Harvard Econ. Stud. Harvard Univ. Press, Cambriage, Mass.

Cook, H. L., and G. H. DAY.

1947. The dry milk industry. 169 p. Amer. Dry Milk Inst., Inc., Chicago, Ill., in coöperation with U. S. Dept. Agr., Bur. Agr. Econ. and Prod. and Market. Admin.

Cowden, J. M., and H. C. Trelogan.

1948. Flexibility of operations in dairy manufacturing plants. U. S. Dept. Agr., Bur. Agr. Econ. and Prod. and Market. Admin. Cir. 799:1-40. Wash., D.C.

Dairy Listing Service.

Dairy and poultry yearbook. Chicago, Ill. (Annual issues.)

Froker, R. K., and C. M. HARdin.

1942. Paying producers for fat and solids-not-fat in milk. Wisconsin Agr. Exp. Sta. Res. Bul. 143:1-48.

GILBERT, S. J.

1945. Wisconsin dairy production, utilization, and related data. Wisconsin Dept. Agr. Crop and Livestock. Report. Serv. Bul. 250:1-111.

Howe, C. B.

1946. Marketing margins and costs for dairy products. U. S. Dept. Agr., Bur. Agr. Econ. Tech. Bul. 936:1-79.

JACK, E. L., F. H. AвBotT, E. B. Roessler, and A. W. Irwin.

1951. Relationship of solids-not-fat in California milk. California Agr. Exp. Sta. Bul. $726: 1-12$

J ACOBSEN, M. S.

1936. Butterfat and total solids in New England farmers' milk as delivered to processing plants. Jour. Dairy Sei., 19:171-76.

Koller, E. F., and O. B. JESNESS.

1937. Organization and operation of Minnesota coöperative creameries. Minnesota Agr. Exp. Sta. Bul. 333:1-82.

Mathis, A. G., and D. E. Hirsch.

1950. Butter pricing by Iowa creameries. U. S. Farm Credit Admin. (in coöperation with Iowa Agr. Exp. Sta.) Cir. C136:1-38. Wash., D.C.

Milk Dealers Association of Metropolitan New York, Inc. Monthly membership letters. New York City. (Entire series.) 
Miller, A. H.

1949. Pricing American cheese at Wisconsin factories. Wisconsin Agr. Exp. Sta. Res. Bul. 163:1-31.

MORTENSEN, M. 1947. Management of dairy plants. 407 p. The Macmillan Company, New York City.

Nicholls, W. H.

1939. Post-war developments in the marketing of cheese. Iowa Agr. Exp. Sta. Res. Bul. $261: 1-148$.

Overman, O. R., O. F. GarretT, K. E. Wright, and F. P. SAUManN.

1939. Composition of milk of brown Swiss cows. (With summary of data on the composition of milk from cows of other breeds.) Illinois Exp. Sta. Bul. 457:575-623.

Potr, L. A.

1950. Transportation rates, products transported and trade barriers important to South Dakota. Univ. South Dakota, School Bus. Admin. Bul. 23:1-110.

Price, W. V., and L. Germain.

1931. Standardization of milk for the manufacture of American cheese. Wisconsin Agr. Exp. Sta. Res. Bul. 108:1-11.

REDER, M. W.

1947. Studies in the theory of welfare economics. 208 p. Columbia Univ. Press, New York City.

STEPP, J. M.

1942. The economics of price in the milk industry. 154 p. Univ. Virginia, Bur. Pub. Admin. Charlottesville, Virginia.

Thomsen, L. C.

1945. Shall we plan toward continued diversification? Amer. Butter Review, Mar., 1945, p. 74. New York City.

U. S. BUREAU AGRICUltural Economics. 1929. Dairy statistics. Statis. Bul. 25:1-256. Wash., D. C.

U. S. Bureau Agricultural Economics.

1934. Summary of dairy situation statisties—production, prices, stocks. 96 p. Wash., D.C.

U. S. Bureau Agricultural EConomics.

1934. Cold storge holdings. Statis. Bul. 48:1-43.

U. S. BUREAU Agricultural Economics.

1937. Dry or powdered milk statistics. 20 p. Wash., D.C.

U. S. BUREaU Agricultural EConomics.

1950. Changes in the dairy industry, United States, 1920-50.144 p. Wash., D.C.

U. S. BUREAU AGRICUltural EConomics. Production of manufactured dairy products. Wash., D.C. (Annual issues.)

U. S. BUReau Agricultural EConomics.

Farm production, disposition, and income from milk. Wash., D.C. (Annual issues.)

U. S. BUREAU Agricultural ECONOMICS.

Milk prices paid at creameries and cheese factories. Wash., D.C. (Monthly issues.)

U. S. BUREAU AGRICUltural Economics.

Evaporated, condensed, and dry milk report. Wash., D.C. (Monthly issues.)

U. S. DeparTMent AGRICUlture.

1933. Marketing agreement for evaporated milk industry. 16 p. U. S. Agr. Adjust. Admin. Agreement 7, plus amendments. Wash., D.C.

U. S. DePartment AgRiculture.

1935. Marketing agreement and license for evaporated milk industry. 50 p. U. S. Agr. Adjust. Admin. Agreement 60 and License 100. Wash., D.C.

U. S. DEPARTMENT AGRICULTURE.

1946. Milk products-costs, prices, and profits of war food purchases. $72 \mathrm{p}$. Prod. and Market. Admin. Wash., D.C.

U. S. Department AGRICUlture.

Dairy and poultry market statistics. Prod. and Market. Admin. Wash., D.C. (Annual issues.)

U. S. DePARTMEnT Commerce.

Census of manufactures. Bur. of Census. Wash., D.C. (Entire series.) 


\section{U. S. DEPARTMENT INTERIOR.}

1946. Columbia Basin joint investigations-agricultural processing industries. 120 p. Bur. Reclamation Problem 24. Wash., D.C.

\section{U. S. Department LaboR}

Average wholesale prices and index numbers of individual commodities. Bur. Labor Statistics. Wash., D.C. (Annual and monthly issues.)

Wilcox, E. C., O. E. Krause, and L. Brereton.

1950. Utilization of Wisconsin milk. Wisconsin Dept. Agr. in coöperation with U. S. Dept. Agr., Spec. Bul. 3:1-113. 


\section{APPENDIX A Yield Formulas and Their Bases}

\section{Introduction}

In this appendix will be developed specific yield formulas for computing the output of the major product and by-product(s) which can be produced when 100 pounds of milk are utilized in a given operation. Although the procedure is quite simple, it will be written down rather completely in order to dispel any confusion in the mind of the reader.

Milk is composed of fat, nonfat solids, and water. Neglecting certain minute quantities of chemicals that are added to the components of milk which are being processed, it is obvious that the final products must be composed of some combination of the basic parts of milk. In fact, government standards have been established to specify the minimum composition requirements for products moving in interstate trade. Practically all the manufactured dairy products do move between states. From the economic point of view, assuming insufficient demand reaction by consumers to better-thanminimum products, it is to the advantage of a plant to come as near to these minimum requirements as possible. It will be assumed here that the divergence is insignificant in those cases where state laws permit standardization of the raw milk prior to processing. This seems in close accord with fact. Formulas also will be developed for unstandardized operations.

The term standardization refers to the operation of adjusting the composition of the originally received milk so that the fat and nonfat content is in the correct relationship to satisfy minimum product requirements. For simplification, it will be assumed that all standardization is intraplant, instead of being accomplished by securing cream or skim milk from outside sources. The purpose of this is to permit a complete determination of the potential output from 100 pounds of milk within a given plant. It is assumed that interplant diversion of skim milk or cream would only progress to the point at which it was marginally profitable to use these primary by-products within the plant. Both diversions for standardization purposes and for centralizing by-product production are included in this assumption.

By now, it should have been guessed that the estimation of product yields from 100 pounds of milk will be based on composition requirement for products and the composition of the raw milk used. Fortunately, an approximately linear average relationship exists between the nonfat portion of milk and the fat portion, especially for aggregated lots from numerous farmers (Jacobsen, 1936 and Jack, et al., 1951). On the assumption that the actual paired weights of fat and nonfat solids per 100 pounds of milk in all possible aggregate lots delivered to any plant will be symmetrically disposed in a random manner over time around the average relationship, no account will be taken of the statistical dispersion of the potential data. The strong, economic, interproduct relationships will be affected principally by the average relationship instead of the variations therefrom. This assumes, of course, that the variations are temporally unpredictable.

After these introductory remarks, the development proceeds directly. 
Knowledge of an average relationship between nonfat solids and fat in milk allows one to determine yield formulas in terms of the fat content of the milk only. This is particularly useful since testing milk for fat content is more easily accomplished than testing for nonfat.

\section{Definitions (on 100 pounds of milk basis)}

$Q_{\mathrm{N}} \quad=$ Average pounds of nonfat solids in 100 pounds of milk used by a plant.

$F \quad=$ Pounds of fat in 100 pounds of milk used by a plant.

$Q_{40}=$ Pounds of 40 per cent cream.

$Q_{\mathrm{B}} \quad=$ Pounds of skim milk containing .1 per cent fat.

$Q_{\mathrm{b}} \quad=$ Pounds of butter (total for operation).

$Q_{\mathrm{e}} \quad=$ Cases of evaporated milk (43.5 net pounds per case).

$Q_{w} \quad=$ Pounds of whole milk powder.

$Q_{\mathrm{c}} \quad=$ Pounds of American cheddar cheese.

$Q_{\mathrm{wh}}=$ Pounds of dry whey.

$Q_{\mathrm{n}} \quad=$ Pounds of dry buttermilk and skim solids in butter operation.

$Q_{\mathrm{n}-\mathrm{s}}=$ Pounds of dry nonfat solids from skim milk.

$Q_{\text {bu }}=$ Pounds of dry buttermilk.

$Q_{\mathrm{b}-40}=$ Pounds of butter from 40 per cent cream in cheese operation.

$Q_{\mathrm{b}-\mathrm{wh}}=$ Pounds of butter from whey in cheese operation.

\section{General assumptions}

$a$. Average nonfat solids in raw milk are expressed in terms of the fat content by means of Jacobsen's formula, $Q_{\mathrm{N}}=7.07+.4 F$ (Jacobsen, 1936).

$b$. Skim milk contains 0.1 per cent fat.

$c$. The quantity of cream having $k$ per cent fat which can be separated from 100 pounds of raw milk is given by $Q_{\mathrm{k}}=\frac{F-.1}{.01(k-.1)}$.

$d$. The quantity of residual skim milk is given by $Q_{\mathrm{s}}=100-Q_{\mathrm{k}}$.

$e$. Cream and skim milk losses are subsumed in the final product loss allowances.

4. The butter operation

a. 98.5 per cent of the fat in the cream goes into butter, which has a fat content of 80.5 per cent.

$b$. Buttermilk contains fat equivalent to the weight of the nonfat solids lost by occlusion in the butter.

c. In drying skim milk or buttermilk, a loss of solids results, which is equivalent to the gain by moisture retained at about 2 or 3 per cent of total weight. Therefore, $Q_{\mathrm{n}}=$ $Q_{\mathrm{N}}+.001 Q_{\mathrm{B}}$.

d. $Q_{\mathrm{n}-\mathrm{s}}$ is found by evaluating $Q_{\mathrm{n}}$ at $F=3$ and $F=5$, then multiplying by $\frac{Q_{\mathrm{s}}}{100-F}$
as a percentage of skim milk correction. The slope and intercept values are then easily computed for a particular value of " $k$."

$e . Q_{\mathrm{bu}}$ is found by subtraction.

\section{The evaporated milk operation}

a. Composition is 7.9 per cent fat, 18.0 per cent nonfat solids, and 74.1 per cent moisture.

$b$. In general, skim milk or cream is removed (depending on whether $F<3.765$ or $F>3.765$ ) from the raw milk in sufficient quantities so that the ratio of nonfat solids to fat in the residual is equal to 2.278. These functions of $F$ are nonlinear in form, but nearly linear in the relevant range of $3 \leqq F \leqq 5$. Consequently, they have been approximated very closely by linear functions for $F<3.765$ and for $F>3.765$. 
c. A 2 per cent loss of total solids is assumed. The yield of evaporated milk is found by multiplying the residual fat content in pounds by $.98 / .079$ or 12.405 . Conversion to case yields is secured by dividing by 43.5 .

$d$. By-product formulas are computed by proportions of yields experienced in the butter operation. Slopes and intercepts are easily found when quantities are determined at $F=3$, and at $F=5$. Zero by-products result when $F=3.765$.

6. The whole milk powder operation

a. Composition is 27 per cent fat, $\mathbf{7 1}$ per cent nonfat solids, and 2 per cent moisture.

$b$. In general, skim milk or cream is removed (depending on whether $F<3.17$ or $F>3.17$ ) from the raw milk in sufficient quantities so that the ratio of nonfat solids to fat in the residual is equal to 2.630 . These functions of $F$ are nonlinear in form, but nearly linear in the relevant range of $3 \leqq F \leqq 5$. Consequently, they have been approximated very closely by linear functions for $F<3.17$ and for $F>3.17$.

c. A 2 per cent loss of total solids is assumed. The yield of whole milk powder is found by multiplying the residual fat content in pounds by $.98 / .27$ or 3.63 .

$d$. By-product formulas are computed by proportions of yields experienced in the butter operation. Slopes and intercepts are easily found when quantities are determined at $F=3$ and $F=5$. Zero by-products result when $F=3.17$.

\section{The American cheese operation}

a. Composition is 37 per cent moisture and 63 per cent total cheese solids of which 50 per cent is fat and 50 per cent is nonfat solids. The legal maximum moisture content is 39 per cent but 37 was used, since some writers suggested the latter as more representative.

$b$. The quantity of total cheese solids is given by $Q_{\mathrm{C}}=1.45 F+.90$. This is based on 93 per cent of the fat content of the milk entering into the final cheese-making process going into the cheese. This means that $.52 F+.90$ is equal to the nonfat solids which combine with the fat. Upon standardization, sufficient cream must be removed so that 93 per cent of the residual fat is equal to the residual nonfat cheese solids. Rectification of the functions of $F$ which resulted gave good linear approximations in the range of $3 \leqq F \leqq 5$.

$c$. A 2 per cent loss of total solids is assumed. The yield of cheese is found by multiplying the residual fat content in pounds by $\frac{(.93)(.98)}{.315}$ or 2.89 .

$d$. Slopes and intercepts for $Q_{\mathrm{b}-\mathrm{k}}$ and $Q_{\mathrm{bu}}$ are found by evaluating proportions to yields in butter operation at $F=3$ and at $F=5$, and then passing the yield line through these points.

$e$. Whey butter yield is determined by separation of the residual fat in the whey and churning. Separation is at 40 per cent cream.

$f$. Whey solids are computed as a residual of total nonfat solids, that is, by computing $Q_{\mathrm{wh}}=Q_{\mathrm{N}}-\frac{.315}{.98} Q_{\mathrm{c}}-Q_{\mathrm{bu}}$, at $F=3$ and $F=5$, and then passing the yield line through these points.

8. Approximate equations for yields in pounds of products from 100 pounds of milk having a fat content of $F$ pounds, when standardizing is practiced

Included in the formulas under each operation are both the primary separation yields and the final product yields. For instance, $Q_{40}$ and $Q_{s}$ are given for the butter operation as well as the final yield equations upon processing these primary components. Also certain partial yield formulas have been added together to secure totals in a given operation. 
$a$. The butter operation with dry buttermilk and nonfat solids as by-products.

For all values of $F$

Separation of 40 per cent cream

$Q_{40}=2.5063 F-.2506$

$Q_{\mathrm{B}}=100.2506-2.5063 \mathrm{~F}^{\prime}$

$Q_{\mathrm{b}}=1.2267 F-.1227$

$Q_{\mathrm{n}-\mathrm{s}}=7.3026+.2307 F$

$Q_{\text {bu }}=.1668 F-.1323$

$Q_{\mathrm{n}}=7.1703+.3975 F$

$b$. The evaporated milk operation

(1) For $F \leqq 3.765$ with nonfat solids as by-product

Standardization by removal of skim milk

$Q_{s}=85.1703-22.6216 \mathrm{~F}$

$Q_{\mathrm{n}-\mathrm{8}}=7.3418-1.9500 F$

$Q_{\mathrm{c}}=.2916 \mathrm{~F}-.0243$ (cases)

(2) For $F \geqq 3.765$ with butter and dry buttermilk as by-products

Standardization by removal of 40 per cent cream

$Q_{10}=2.1999 F-8.2826$

$Q_{\mathrm{b}}=1.0767 F-4.0538$

$Q_{\text {bu }}=.1257 F-.4733$

$Q_{\text {e }}=.0342 F+.9448$ (cases)

c. The American cheddar cheese operation with butter, dry buttermilk, and dry whey as by-products

For all values of $F$

Standardization by removal of 40 per cent cream

$Q_{40}=1.0408 F-1.9172$

$Q_{\mathrm{h} \rightarrow 0}=.5094 F-.9384$

$Q_{\mathrm{bu}}=.0634 F-.1292$

$Q_{\mathrm{c}} \quad=1.7157 F+2.2163$

$Q_{\mathrm{wh}}=6.4868-.2149 \mathrm{~F}$

$Q_{\mathrm{b}-\mathrm{wh}}=.0546 F-.0577$

$Q_{\mathrm{b}} \quad=.5640 F-.9961$

$d$. The whole milk powder operation

(1) For $F \leqq 3.17$ with dry nonfat solids as by-products

Standardization by removal of skim milk

$Q_{8}=85.2384-26.8391 F$

$Q_{\mathrm{n}-\mathrm{s}}=7.3500-2.3186 F^{\prime}$

$Q_{\mathrm{w}}=3.7233 F^{\prime}-.2958$

(2) For $F \geqq 3.17$ with butter and dry buttermilk as by-products

Standardization by removal of 40 per cent cream

$Q_{40}=2.2414 F-7.1052$

$Q_{\mathrm{b}}=1.0970 F-3.4776$

$Q_{\text {bu }}=.1281 F-.4061$

$Q_{\mathrm{w}}=.3755 F+10.3168$

9. Approximate equations for yields in pounds of products from 100 pounds of milk having a fat content of $F^{\prime}$ pounds, when standardizing is not practiced

a. Evaporated milk operation

(1) $F \leqq 3.765$

$Q_{\mathrm{n}}=.2852 F$ (cases)

(2) $F \geqq 3.765$

$Q_{\mathrm{e}}=.8849+.0690 F$ (cases) 
b. Whole milk powder operation

(1) For $F \leqq 3.17$ Standardizing is necessary in order to secure minimum of 27 per cent fat.

(2) For $F \geqq 3.17$

$Q_{\mathrm{w}}=1.4 F+7.07$

c. American cheddar cheese operation

For all values of $F$

$Q_{\mathrm{c}} \quad=2.2556 F+1.4000$

$Q_{\mathrm{b}-\mathrm{wh}}=.0887 F-.1083$

$Q_{\mathrm{wh}}=6.1700-.1200 F$

\section{APPENDIX B \\ Processing and Other Cost Allowances}

In this appendix are presented the bases used in estimating average total unit processing costs. These costs are the monetary estimates of the long-run real costs involved in the processing activities. Since no continuous series of microeconomic studies have been made to give detailed information for this purpose, a rather arbitrary procedure has been employed. It is believed that accuracy sufficient for present purposes has been achieved by the crude method used. For aggregative analyses similar to those made in this paper, it was not considered reasonable to worry about variations in costs due to seasonality of production, interplant differences in scale, or localized cases of low or high factor prices. Competition tends to result in uniform supply prices for services with firms absorbing losses or gains due to the above factors. An attempt was made, however, to have the estimated cost allowances represent the monetary valuations of real costs for optimum long-run plants.

The basic procedure employed was to utilize a set of costs for the year 1943 and, with reasonable price indexes, move the components of these costs to make other estimates for other years. The following tables and quoted sources should clarify the issue. Only annual estimates were made and applied for each month of the year.

\section{To Estimate Historical Cost Allowances (Based on 1943 data in Table 16)}

1. Packaging costs
(a) Butter-Moved by column G of table 17, 1921-1935.
Moved by column $\mathrm{E}$ of table 17, 1936-1950.
(b) Cheese-Moved by column I of table 17, 1921-1950.
(c) Evaporated milk-Moved by column D of table 17, 1921-1950.
(d) All dry products-Moved by column J of table 17, 1921-1950.

2. Labor costs

(a) All labor costs were moved by appropriate labor indexes given in table 18 .

3. Plant, general, administrative, and selling costs

(a) This cost category was moved by column $\mathrm{H}$ of table 17 .

4. Receiving, testing, and separating or standardizing costs

(a) This cost eategory was moved by a price index which was the simple average of column $\mathrm{H}$ of table 17 and either column $\mathrm{A}$ or $\mathrm{B}$ in table 18, depending on whether the reference area was Midwest or West. 
TABLE 16

PROCESSING COSTS IN 1943, BY PROCESSES AND AREAS

\begin{tabular}{|c|c|c|c|c|c|}
\hline \multirow[b]{2}{*}{ Process } & \multirow[b]{2}{*}{ Geographic area } & \multicolumn{4}{|c|}{ Costs } \\
\hline & & Package & Labor & $\begin{array}{c}\text { Plant, } \\
\text { general } \\
\text { adminis- } \\
\text { trative, } \\
\text { and selling }\end{array}$ & Total \\
\hline \multirow[b]{2}{*}{ Making and packaging butter........ } & \multirow[b]{2}{*}{$\begin{array}{l}\text { Midwest............ } \\
\text { West (Idaho) ...... }\end{array}$} & \multicolumn{4}{|c|}{ cents per pound } \\
\hline & & $\begin{array}{r}0.26 \\
28\end{array}$ & $\begin{array}{l}0.80 \\
0.82\end{array}$ & $\begin{array}{l}1.64 \\
1.49\end{array}$ & 2.70 \\
\hline Making and packaging American cheese. & $\begin{array}{l}\text { Midwest........... } \\
\text { West (Oregon)...... }\end{array}$ & $\begin{array}{l}.91 \\
.94\end{array}$ & $\begin{array}{l}1.30 \\
1.41\end{array}$ & $\begin{array}{l}1.48 \\
1.50\end{array}$ & $\begin{array}{l}3.69 \\
3.85\end{array}$ \\
\hline Drying and packaging skim milk...... & $\begin{array}{l}\text { Midwest........... } \\
\text { West (Calif.) ...... }\end{array}$ & $\begin{array}{r}.80 \\
0.84\end{array}$ & $\begin{array}{l}0.67 \\
0.80^{*}\end{array}$ & $\begin{array}{l}1.66 \\
1.99^{*}\end{array}$ & $\begin{array}{l}3.13 \\
3.63\end{array}$ \\
\hline \multirow[t]{2}{*}{ Dryingt and packaging whole milk.... } & \multirow[t]{2}{*}{ Midwest and West.. } & 2.00 & 1.81 & 3.07 & 6.88 \\
\hline & & \multicolumn{4}{|c|}{ dollars per 100 pounds } \\
\hline \multirow[t]{2}{*}{$\begin{array}{l}\text { Receiving, testing, and separating or } \\
\text { standardizing milk } \ldots \ldots \ldots \ldots \ldots \ldots\end{array}$} & \multirow[t]{2}{*}{ Midwest and West.. } & $\ldots$ & $\ldots$ & $\ldots$ & $.10 \ddagger$ \\
\hline & & \multicolumn{4}{|c|}{ dollars per case } \\
\hline Evaporating and packaging milk...... & $\begin{array}{l}\text { Midwest........ } \\
\text { West (Calif.)... }\end{array}$ & $\begin{array}{l}0.713 \\
0.753\end{array}$ & $\begin{array}{l}0.163 \\
0.190\end{array}$ & $\begin{array}{l}0.264 \\
0.299\end{array}$ & $\begin{array}{l}1.140 \\
1.252\end{array}$ \\
\hline
\end{tabular}

* Adjusted to be comparable to Midwest. Original differences assumed to be due to accounting variations. + Based on a 50-pound can in a plywood drum.

$\ddagger$ Estimated for 1943 from minimum value of .12 for 1945 as given by Thomsen, L. C. "Shall We Plan Toward Continued Diversification?" American Butter Review, March, 1945.

Source: U. S. Dept. Agr. Production and Marketing Administration, Compliance and Investigation Branch, Milk Products-Costs, Prices and Products of War Food Purchases. Wash., D.C., 1946. 


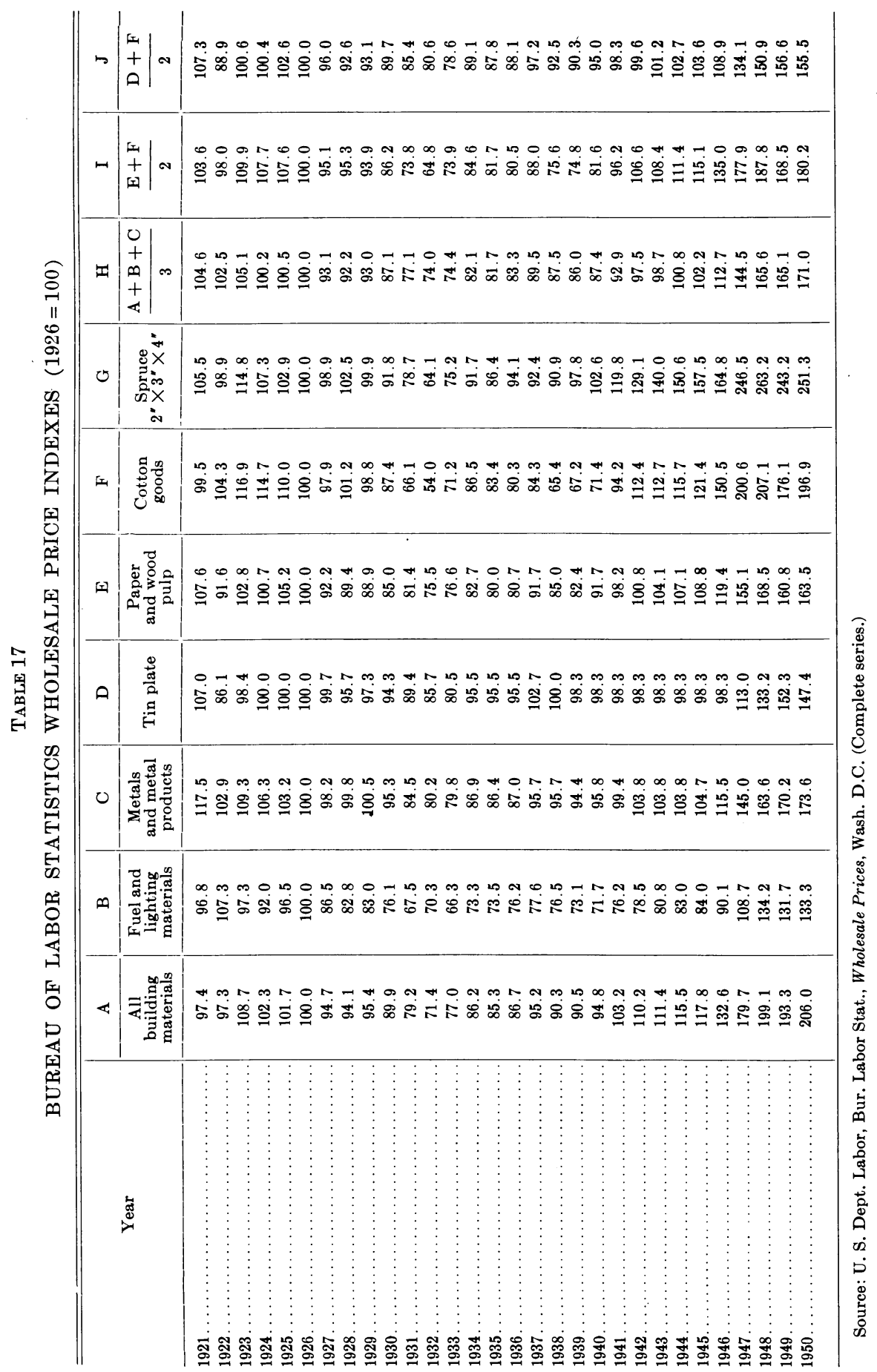




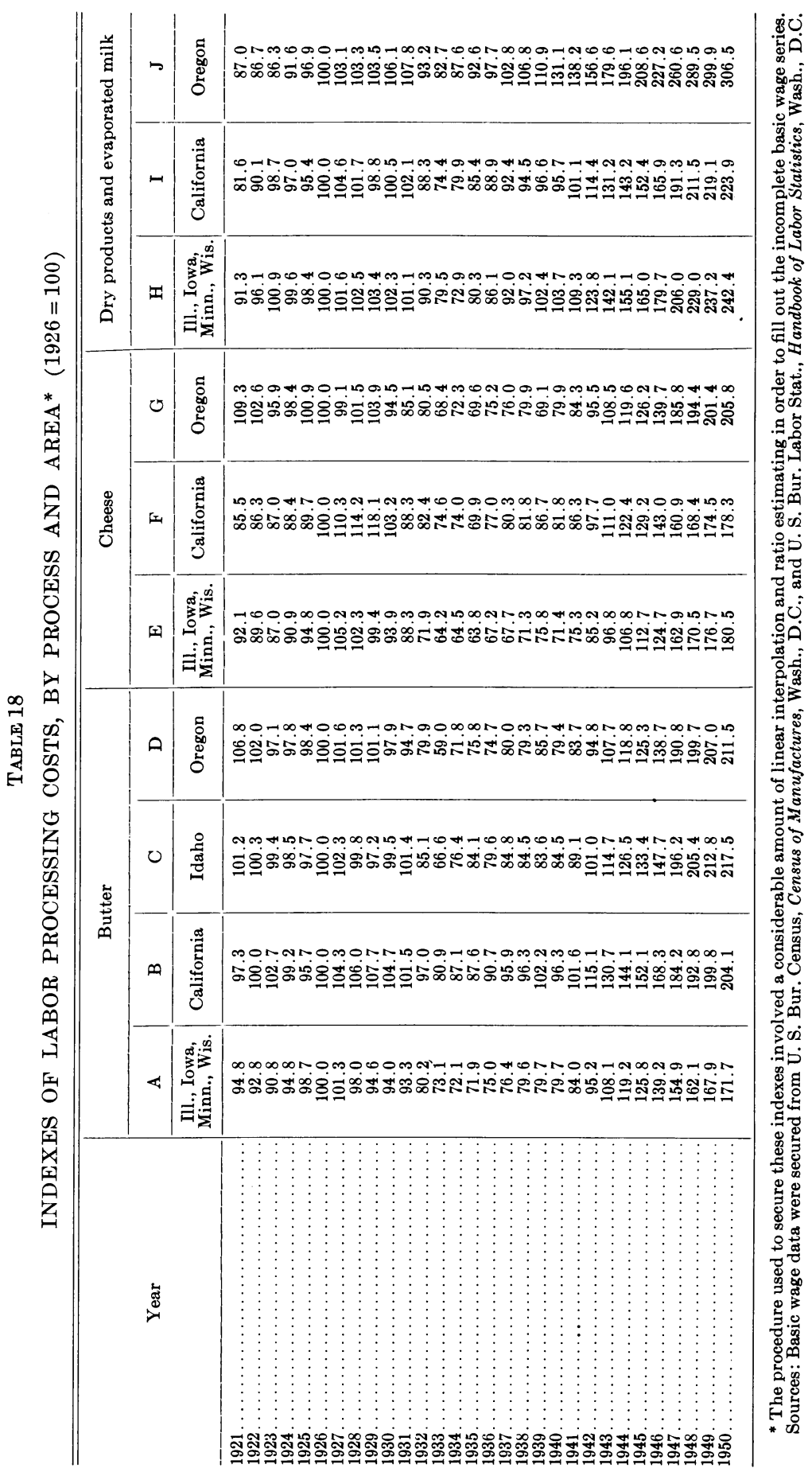


TABLE 19

ESTIMATED AVERAGE TOTAL UNIT PROCESSING COST ALLOWANCES (for Illinois, Iowa, Minnesota, and Wisconsin)

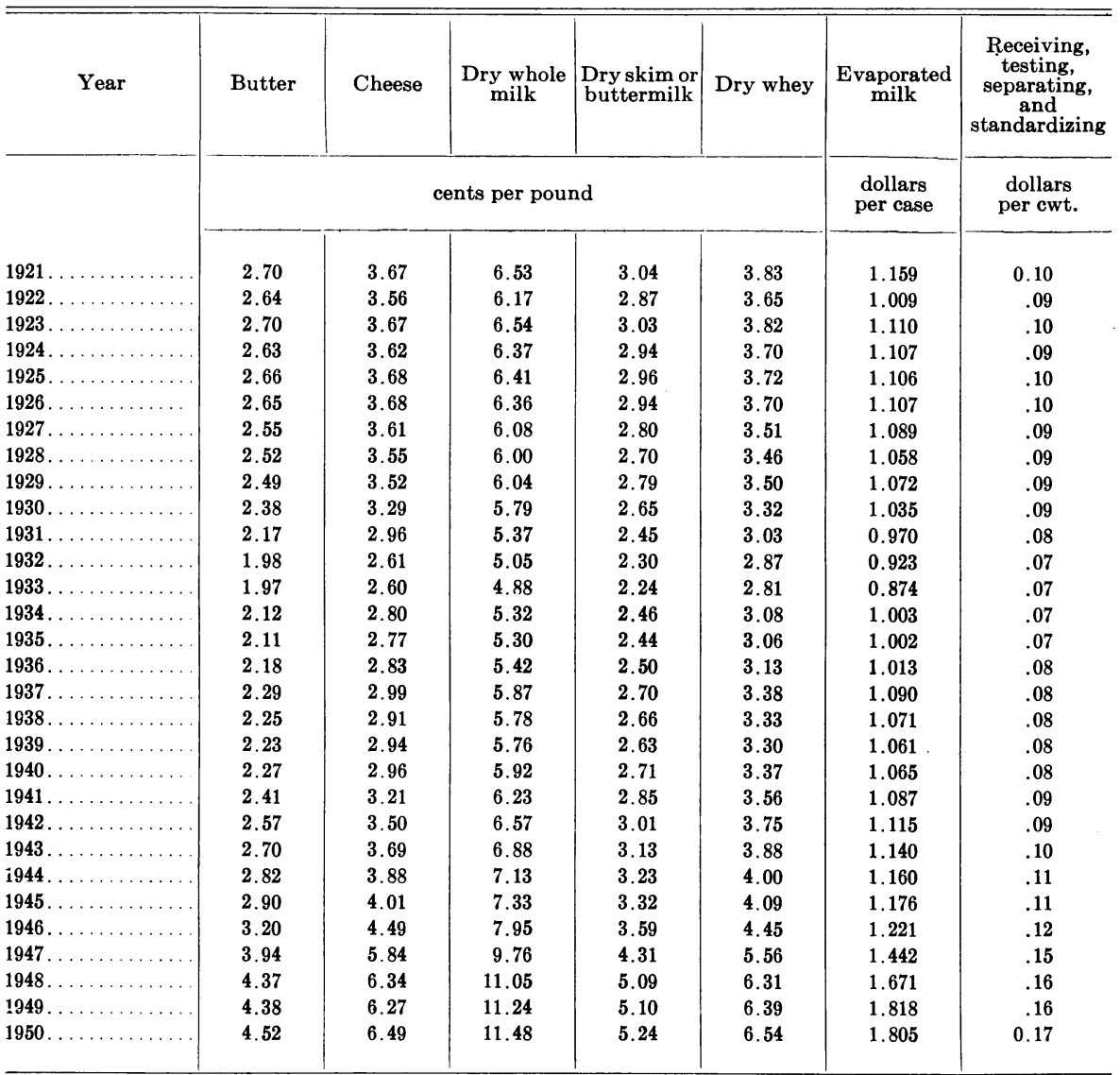

Source: Computed from other tables in Appendix B, as stated on pages 328-29. 


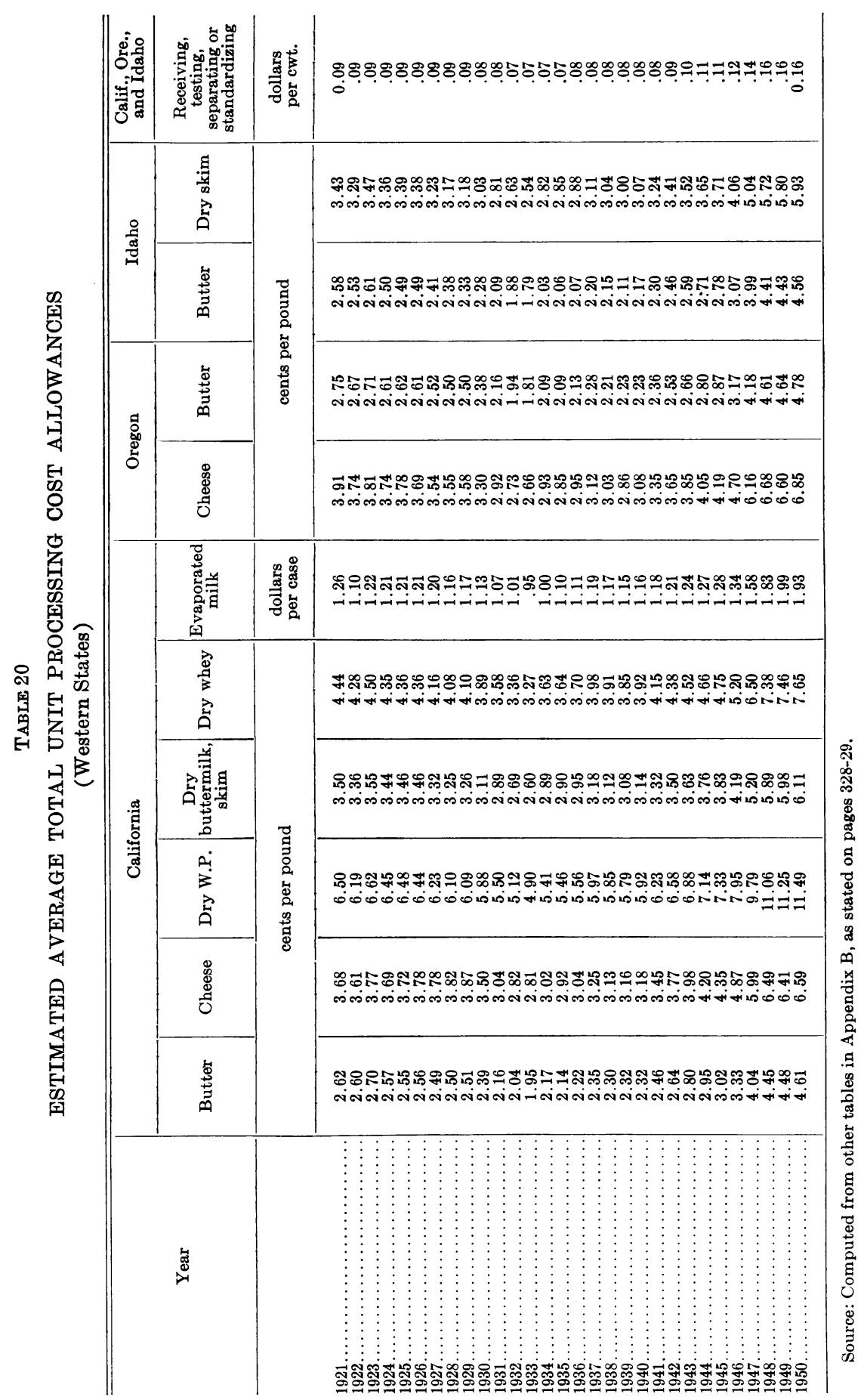


TABLE 21

ESTIMATED AVERAGE FREIGHT,* HANDLING, AND STORAGE COST ALLOWANCE

\begin{tabular}{|c|c|c|c|c|c|}
\hline \multirow{2}{*}{ Year } & \multicolumn{2}{|c|}{$\begin{array}{l}\text { Illinois, Jowa, Minnesota, } \\
\text { Wisconsin }\end{array}$} & \multirow{2}{*}{$\begin{array}{c}\text { California } \\
\begin{array}{c}\text { Butter and } \\
\text { cheese }\end{array}\end{array}$} & \multirow{2}{*}{$\underbrace{\text { Oregon }}_{\begin{array}{c}\text { Butter and } \\
\text { cheese }\end{array}}$} & \multirow{2}{*}{$\begin{array}{l}\text { Idaho } \\
\text { Butter }\end{array}$} \\
\hline & Butter & Cheese & & & \\
\hline & \multicolumn{5}{|c|}{ cents per pound } \\
\hline $1921 \ldots \ldots \ldots \ldots \ldots \ldots \ldots \ldots$ & 0.80 & 0.70 & 0.50 & 0.70 & 1.00 \\
\hline $1922 \ldots \ldots \ldots \ldots \ldots \ldots \ldots \ldots$ & .70 & .60 & .45 & .60 & 0.90 \\
\hline $1923 \ldots \ldots \ldots \ldots \ldots \ldots$ & .70 & .60 & .45 & .60 & .90 \\
\hline $1924 \ldots \ldots \ldots \ldots \ldots \ldots \ldots$ & .70 & .60 & .45 & .60 & .90 \\
\hline $1925 \ldots \ldots \ldots \ldots \ldots \ldots \ldots$ & .70 & .60 & .45 & .60 & .90 \\
\hline $1926 \ldots \ldots \ldots \ldots \ldots \ldots \ldots$ & .70 & .60 & .45 & .60 & .90 \\
\hline $1927 \ldots \ldots \ldots \ldots \ldots \ldots \ldots \ldots$ & .70 & .60 & .45 & .60 & .90 \\
\hline $1928 \ldots \ldots \ldots \ldots \ldots \ldots \ldots \ldots$ & .70 & .60 & .45 & .60 & .90 \\
\hline $1929 \ldots \ldots \ldots \ldots \ldots \ldots$ & .70 & .60 & .45 & .60 & .90 \\
\hline $1930 \ldots \ldots \ldots \ldots \ldots \ldots \ldots$ & .70 & .60 & .45 & .60 & .90 \\
\hline $1931 \ldots \ldots \ldots \ldots \ldots \ldots \ldots$ & .70 & .60 & .45 & .60 & .90 \\
\hline $1932 \ldots$ & .65 & .55 & .40 & .55 & .80 \\
\hline $1933 \ldots \ldots \ldots$ & .65 & .55 & .40 & .55 & .80 \\
\hline $1934 \ldots \ldots \ldots$ & .65 & .55 & .40 & .55 & .80 \\
\hline $1935 \ldots \ldots \ldots$ & .65 & .55 & .40 & .55 & .80 \\
\hline $1936 \ldots \ldots \ldots \ldots \ldots \ldots \ldots \ldots$ & .65 & .55 & .40 & .55 & .80 \\
\hline $1937 \ldots \ldots \ldots$ & .65 & .55 & .40 & .55 & .80 \\
\hline $1938 \ldots \ldots \ldots$ & .70 & .60 & .45 & .60 & .90 \\
\hline$\ldots \ldots \ldots \ldots$ & .70 & .60 & .45 & .60 & .90 \\
\hline $1940 \ldots \ldots \ldots$ & .70 & .60 & .45 & .60 & .90 \\
\hline $1941 \ldots \ldots \ldots$ & .70 & .60 & .45 & .60 & .90 \\
\hline $1942 \ldots \ldots \ldots$ & .70 & .60 & .45 & .60 & 0.90 \\
\hline $1943 \ldots \ldots \ldots \ldots \ldots \ldots \ldots \ldots$ & .75 & .65 & .50 & .65 & 1.00 \\
\hline $1944 \ldots \ldots \ldots \ldots \ldots \ldots \ldots$ & .75 & .65 & .50 & .65 & 1.00 \\
\hline $1945 \ldots \ldots \ldots \ldots \ldots \ldots \ldots \ldots$ & .75 & .65 & .50 & .65 & 1.00 \\
\hline $1946 \ldots \ldots$ & .80 & .70 & .55 & .70 & 1.05 \\
\hline$\ldots \ldots \ldots \ldots \ldots$ &. .80 & .70 & .55 & .70 & 1.05 \\
\hline $1948 \ldots \ldots \ldots \ldots \ldots \ldots \ldots \ldots$ & .80 & .70 & .55 & .70 & 1.05 \\
\hline $1949 \ldots \ldots \ldots$ & 0.90 & .80 & .60 & .80 & 1.15 \\
\hline $1950 \ldots \ldots \ldots \ldots \ldots \ldots \ldots \ldots$ & 1.00 & 0.88 & 0.66 & 0.88 & 1.26 \\
\hline
\end{tabular}

* Freight costs are to Chicago for the first two columns and to San Francisco for the last two columns. Handling and storage costs are for temporary (a week or two) storage services required at the consumption point.

Sources: Basic freight rates secured from Dairy and Poultry Yearbook, Dairy Listing Service (Chicago, Ill.) and a Southern Pacific Railroad agency. 
The journal Hilgardia is published at irregular intervals, in volumes of about 600 pages. The number of issues per volume varies.

Subscriptions are not sold. The periodical is sent as published only to libraries, or to institutions in foreign countries having publications to offer in exchange.

You may obtain a single copy of any issue free, as long as the supply lasts; please request by volume and issue number from:

\section{Publications Office \\ College of Agriculture \\ Berkeley 4, California}

The limit to nonresidents of California is 10 separate issues on a single order. A list of the issues still available will be sent on request.

In order that the information in our publications may be more intelligible it is sometimes necessary to use trade names of products or equipment rather than complicated descriptive or chemical identifications. In so doing it is unavoidable in some cases that similar products which are on the market under other trade names may not be cited. No endorsement of named products is intended nor is criticism implied of similar products which are not mentioned. 